\title{
A conjectura de Boyland para homeomorfismos do anel
}

\author{
Bernardo Gabriel Marques
}

\author{
DisSERTAÇÃO APRESENTADA \\ AO \\ Instituto De Matemática e EstatísticA \\ DA \\ Universidade DE SÃo PAUlo \\ PARA \\ OBTENÇÃO DO TÍTULO \\ $\mathrm{DE}$ \\ Mestre em CiÊnCIAS \\ Programa: Matemática Aplicada \\ Orientador: Prof. Dr. Fabio Armando Tal
}

Durante o desenvolvimento deste trabalho o autor recebeu auxílio financeiro da CNPq

São Paulo, abril de 2011 


\section{A conjectura de Boyland para homeomorfismos do anel}

Esta versão definitiva da dissertação contém as correções e alterações sugeridas pela Comissão Julgadora durante a defesa realizada por Bernardo Gabriel Marques em 14/04/2011.

Comissão Julgadora:

- Prof. Dr. Fabio Armando Tal (orientador) - IME-USP

- Prof. Dr. Salvador Addas Zanata - IME-USP

- Prof. Dr. Bernardo Paschoarelli Veiga Gomes - ITAU 


\section{Agradecimentos}

A mis padres, María Otilia y Juan, por acompañarme siempre y estar presentes en todo momento a pesar de la distancia, a mi hermana, Mariela, y al resto de mi familia por, a su manera, apoyarme y acompañarme.

Eu quero agradecer a toda as pessoas que eu conheci aqui, e que foram e são parte da minha vida (e uma grande ajuda e apoio muitas veces): Sebastian, Pablo, Paula, Cristian, Sandra, Aleja. Sem esquecer das pessoas da sala de estudos, Gustavo, Dylene, Diego, os Brunos, Cleber, Henry e tudas as pessoas da sala. Também agradeço a meu orientador, não só por orientarme neste mestrado, também por darme a liberdade na forma de trabalhar e ajudar na minha adaptação.

Muito obrigado a todos. 


\section{Resumo}

Seja $f$ um homeomorfismo do anel $A=\mathbf{S}^{1} \times[0,1], p: \widetilde{A} \rightarrow A$ a projeção do recobrimento universal $\widetilde{A}=\mathbf{R} \times[0,1]$ e $\mu$ a medida de Lebesgue. Dizemos que $f$ é irrotacional ([3]) se ele preserva área, orientação, as componentes de fronteira e, sendo $\widetilde{f}$ um levantamento de $f$ ao recobrimento universal e $p_{1}: \widetilde{A} \rightarrow \mathbf{R}$ a projeção sobre a primeira coordenada, a função $\phi: A \rightarrow \mathbf{R}$ definida como $\phi(x, y)=p_{1} \circ \widetilde{f}(\widetilde{x}, \widetilde{y})-\widetilde{x}$, para todo $(\widetilde{x}, \widetilde{y}) \in p^{-1}(x, y)$ satisfaz $\int_{A} \phi(x, y) d \mu=0$.

A ideia deste trabalho é apresentar a conjetura de Boyland para o anel e mostrar algums resultados nessa direção. Tal conjectura diz que: Dado um homeomorfismo irrotacional do anel, que possui uma medida com número de rotação positivo, é verdade que, neste caso, existem pontos com número de rotação negativo? Para dar uma resposta parcial a esta pregunta, nesta dissertação (baseada no estudo do [7]) começamos considerando os homeomorfismos do anel que preservam orientação, as componentes de fronteira, com número de rotação positivos em ambas fronteiras, e que tem un levantamento transitivo (o motivo desta hipoteses vem de [3]), mostrando que neste caso 0 está no interior do conjunto de rotação. Este resultado vai permitir provar a conjetura para os homeomorfismos do anel irrotacionais, sem pontos fixos na fronteira e com um levantamento transitivo. Além disso vai permitir estudar a dinâmica de tais homeomorfismos. No final do trabalho, estendemos algums dos teoremas provados ao longo dos capítulos anteriores a um conjunto maior de homeomorfismos e estudamos o comportamento de tais homeomorfismos com base nestes resultados.

Palavras-chave: Homeomorfismos do anel, transitividade, conjunto de rotação, Conjetura de Boyland. 


\section{Abstract}

Let $f$ be a homeomorphism of the annulus $A=\mathbf{S}^{1} \times[0,1], p: \widetilde{A} \rightarrow A$ the covering mapping and $\mu$ the Lebesgue measure. We say that $f$ is a rotationless homeomorphism ([3]) if it preserves Lebesgue, orientation, boundary components and, if $\widetilde{f}$ is a lift of $f$ to $\widetilde{A}$ and $p_{1}: \widetilde{A} \rightarrow \mathbf{R}$ the projection on the first coordinate, the function $\phi: A \rightarrow \mathbf{R}$ defined as $\phi(x, y)=p_{1} \circ \widetilde{f}(\widetilde{x}, \widetilde{y})-\widetilde{x}$, for all $(\widetilde{x}, \widetilde{y}) \in p^{-1}(x, y)$ satisfies $\int_{A} \phi(x, y) d \mu=0$.

The idea of this work is to present Boyland's Conjecture for the annulus and show some results in its direction. The conjecture is the following: Given a homeomorphism of the annulus, which has a measure with positive rotation number, is it true that, in this case, there are points with negative rotation number?. To give a partial answer to this question, in this dissertation (based on [7]) we begin considering the homeomorphisms of the annulus that preserve orientation and boundary components, with positive rotation numbers in the boundaries, with has a transitive lift (the reason for this hypothesis is in [3]), and we show that 0 is in the interior of the rotation set. This result will be of help to prove the Boyland's Conjecture for rotationless homeomorphisms of the annulus, without fixed points in the boundaries and with a transitive lift. In addition, we will be able to study the dynamics of such homeomorphisms. In the end of this work, we extend some of the theorems proved in the previous chapters to a bigger set of homeomorphisms and we study the behavior of such homeomorphisms using these results.

Keywords: Homeomorphisms of the Annulus, transitivity, rotation set, Boyland's Conjecture. 


\section{Sumário}

1 Introdução $\quad 1$

2 Apresentação dos resultados principais $\quad 5$

2.1 Notações, definições e resultados principais . . . . . . . . . . . . . . . . . . 5

3 Preliminares. 11

3.1 Ferramentas básicas . . . . . . . . . . . . . . . . . . . . . 11

4 O conjunto $B^{-}$. $\quad 17$

4.1 Demonstração do Teorema $1 \ldots \ldots \ldots$. . . . . . . . . . . . . . . 17

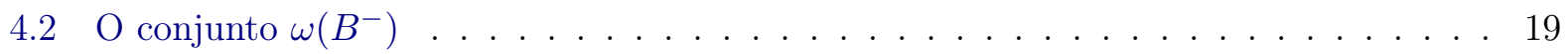

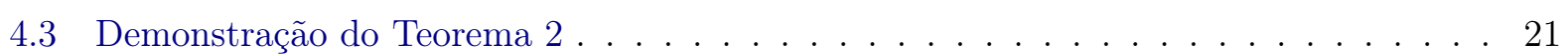

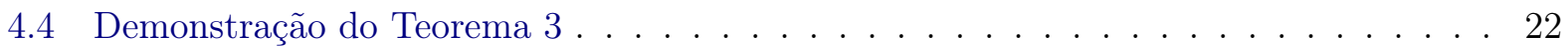

4.5 Demonstração do Corolário $1 \ldots \ldots \ldots$. . . . . . . . . . . . . . . . . 23

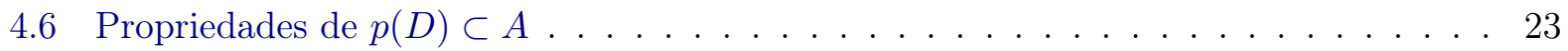

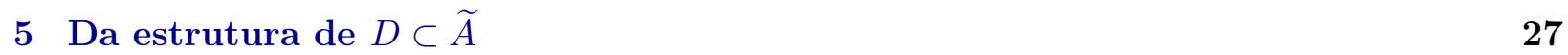

5.1 Resultados preliminares . . . . . . . . . . . . . . . . . . . 27

$5.2 \Gamma \subset D$, componente conexa, e $\left.p\right|_{\Gamma}$ não injetora. . . . . . . . . . . . 34

$5.3 \Gamma \subset D$, componente conexa, e $\left.p\right|_{\Gamma}$ injetora. . . . . . . . . . . . . 35

5.4 Demonstração do Teorema $4 \ldots \ldots \ldots$. . . . . . . . . . . . . 37

$5.4 .1 \Gamma_{1}$ é uma componente injetora . . . . . . . . . . . . . . . . 39

$5.4 .2 \Gamma_{1}$ é uma componente não injetora. . . . . . . . . . . . . . . . . . . 41

5.5 Demonstração do Teorema $5 \ldots \ldots \ldots$. . . . . . . . . . . . . . . . . 44

5.6 Demonstração do Teorema $6 \ldots \ldots \ldots$. . . . . . . . . . . . . . . 46

$6 \quad \mathrm{O}$ conjunto $H_{+}^{\text {inst }} \quad 49$

6.1 Resultados preliminares . . . . . . . . . . . . . . . . . . . . 4 49

6.2 Demonstração do Teorema $7 \ldots \ldots \ldots$. . . . . . . . . . . . . . 52

6.3 Demonstração do Corolário $2 \ldots \ldots \ldots \ldots \ldots \ldots$. . . . . . . . . . . . 54

6.4 Demonstração do Teorema $8 \ldots \ldots \ldots \ldots$. . . . . . . . . . . . 55

6.5 Demonstração do Corolário $3 \ldots \ldots \ldots \ldots \ldots \ldots$. . . . . . . . . . . . . . . . . . . . . . . . 58

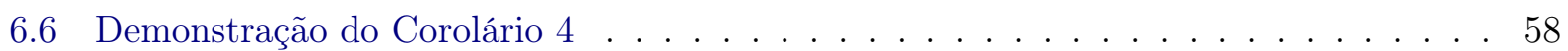

Referências Bibliográficas $\quad 59$ 


\section{Capítulo 1}

\section{Introdução}

Neste trabalho vamos estudar, em termos gerais, a dinâmica dos homeomorfismos do anel $f$ : $A \rightarrow A$, onde $A=\mathbf{S}^{1} \times[0,1]$, onde tais homeomorfismos satisfazem certas condições que serão definidas no final deste capítulo.

No começo do estudo da dinâmica dos homeomorfismos do anel, um objetivo importante foi geralizar certos conceitos e resultados que são conhecidos para os homeomorfismos do círculo. A dinâmica destes homeomorfismos é bem comprendida e tem-se uma quantidade importante de resultados acerca do seu comportamento, tais resultado são apresentados abaixo.

Uma das questões importantes no estudo da dinâmica dos homeomorfismos do círculo ocorre quando um tal homeomorfismo $f$ é equivalente a uma rotação. Para trabalhar neste caminho, a idéia foi trabalhar com o recobrimento universal do círculo, $\mathbf{R}$, e a projeção do recobrimento universal sobre $\mathbf{S}^{1}, p: \mathbf{R} \rightarrow \mathbf{S}^{1}=\mathbf{R} / \mathbf{Z}$. O passo seguinte é trabalhar com um levantamento do homeomorfismo $f$, que chamaremos $F: \mathbf{R} \rightarrow \mathbf{R}$, e satisfaz

$$
f \circ p=p \circ F
$$

É conhecido que tal levantamento é único no sentido que se existe outra $F_{1}$ satisfazendo o anterior, então $F$ e $F_{1}$ diferem por uma constante inteira.

Dizer que $f: \mathbf{S}^{1} \rightarrow \mathbf{S}^{1}$ preserva orientação é pedir que $F$ satisfaça que $F(x+1)=F(x)+1$ para qualquer $x$ real, logo se $f$ preserva orientação, define-se

$$
\widetilde{\rho}(F):=\lim _{|n| \rightarrow \infty} \frac{1}{n}\left(F^{n}(x)-x\right)
$$

e tem-se que este número existe para qualquer $x$ real e independe da escolha do $x$, sendo, além disso, único no sentido que se eu tivesse levantamento $F_{1}, \widetilde{\rho}(F)$ e $\widetilde{\rho}\left(F_{1}\right)$ diferem por um inteiro.

Define-se então o número de rotação do homeomorfismo que preserva orientação $f$ como

$$
\rho(f):=\widetilde{\rho}(F)(\bmod 1)
$$

onde $F$ é um levantamento de $f$.

A questão quando $\rho(f)$ é racional u irracional está bem estudada e tem-se muitos resultados, mas para motivar nosso trabalho, só vamos definir o seguinte:

Definição: Dizemos que $f$ tem uma órbita periódica, se existe $x \in S^{1}$ e $q$ inteiro positivo tal que $f^{q}(x)=x$.

Logo temos um resultado importante que fala o seguinte:

Resultado 1.1 : Seja um homeomorfismo $f: \mathbf{S}^{1} \rightarrow \mathbf{S}^{1}$ que preserva orientação. Então $\rho(f)$ é racional se, e somente se, $f$ tem uma órbita periódica.

E também, temos 
Resultado 1.2 : Seja $F$ um levantamento de $f$, então para todo $x \in \mathbf{R}$, e para todo $n$ inteiro positivo vale

$$
\left|F^{n}(\widetilde{x})-\widetilde{x}-\widetilde{\rho}(F) n\right| \leq 1
$$

Este último resultado mostra que todas as órbitas, acompanham o número de rotação.

O estudo dos homeomorfismos do anel mostra que pode-se geralizar a maioria dos conceitos anteriores para tais homeomorfismos.

Para começar com a geralização, vamos trabalhar no anel $A=\mathbf{S}^{1} \times[0,1]$ e os homeomorfismos $f$ de $A$ nele mesmo. Vamos tomar o recobrimento universal do anel $\widetilde{A}=\mathbf{R} \times[0,1]$ e $p: \widetilde{A} \rightarrow A$ a projeção do recobrimento universal sobre o anel. Trabalharemos também com $p_{1}: \widetilde{A} \rightarrow \mathbf{R}$ a projeção sobre a primeira coordenada, e escolhemos $\widetilde{f}: \widetilde{A} \rightarrow \widetilde{A}$ o levantamento do homeomorfismo $f$ que satisfaz

$$
f \circ p=p \circ \tilde{f}
$$

Para generalizar o número de rotação, vamos definir, em princípio, um conjunto maior, denotado por $\rho(\widetilde{f})$, chamado conjunto de rotação de $\widetilde{f}$, como todos os pontos de acumulação do conjunto

$$
\left\{\frac{p_{1}\left(\widetilde{f}^{n}(\widetilde{z})\right)-p_{1}(\widetilde{z})}{n}, \widetilde{z} \in \widetilde{A}, n \in \mathbf{N}\right\}
$$

O anterior significa que se $\omega \in \rho(\widetilde{f})$ então existem $\widetilde{x}_{k} \in \widetilde{A}, n_{k} \in \mathbf{N}, k \in \mathbf{N}$ tais que

$$
\omega=\lim _{k \rightarrow \infty} \frac{p_{1}\left(\widetilde{f}^{n_{k}}\left(\widetilde{x_{k}}\right)\right)-p_{1}\left(\widetilde{x_{k}}\right)}{n_{k}}
$$

e se existe

$$
\rho(x)=\lim _{n \rightarrow \infty} \frac{p_{1}\left(\widetilde{f}^{n}(\widetilde{x})\right)-p_{1}(\widetilde{x})}{n} \quad x \in A
$$

para $\widetilde{x} \in p^{-1}(x)$ dizemos que $\rho(x)$ é o número de rotação de $x$. Sabemos também que $\rho(\widetilde{f})$ é um intervalo fechado e limitado.

Para posterior trabalho, vamos lembrar que se $f: A \rightarrow A$ é um homeomorfismo e $\mu$ é uma medida boreliana, definida em $A$, dizemos que $\mu$ é $f$-invariante, se

$$
\mu\left(f^{-1}(U)\right)=\mu(U)
$$

para qualquer $U$ subconjunto $\mu$-mensurável de $A$.

Agora se existe uma medida boreliana $\mu \mathrm{em} A f$-invariante, podemos definir o chamado número de rotação de $\mu$ como segue. Seja $\phi: A \rightarrow \mathbf{R}$ definida como

$$
\phi(x)=p_{1}(\widetilde{f}(\widetilde{x}))-p_{1}(\widetilde{x})
$$

para qualquer $\widetilde{x} \in p^{-1}(x)$. Então, definimos o número de rotação de $\mu$ como

$$
\rho(\mu):=\int_{A} \phi(x) d \mu
$$

Do Teorema Ergódico de Birkhoff sai que, por ser $\mu f$-invariante, temos que para quase todo ponto $x \in A$ e para todo $\widetilde{x} \in p^{-1}(x)$

$$
\rho(x)=\lim _{n \rightarrow \infty} \frac{1}{n} \sum_{i=0}^{n-1} \phi \circ f^{i}(x)=\lim _{n \rightarrow \infty} \frac{p_{1}\left(\widetilde{f}^{n}(\widetilde{x})\right)-p_{1}(\widetilde{x})}{n}
$$

existe e

$$
\int_{A} \rho(x) d \mu=\rho(\mu)
$$


Também é conhecido que dado $\omega \in \rho(\widetilde{f})$, existe (pode-se construir) uma medida boreliana $f$-invariante $\tilde{\mu}$ tal que

$$
\omega=\int_{A} \phi d \widetilde{\mu}=\rho(\widetilde{\mu})
$$

Definição: Dizemos que $f$ é um homeomorfismo irrotacional ([4]), se ele preserva a medida de Lebesgue, orientação, as componentes de fronteira e, além disso $\rho(l e b)=0$, onde leb é a medida de lebesgue.

Feita esta introdução e dada a última definição, estamos em condições de apresentar a motivação principal deste trabalho, que é a seguinte conjectura:

Conjectura de Boyland: Dado um homeomorfismo do anel $f$, que preserva área, orientação e as componentes de fronteira, por ([1]), se existem duas medidas de probabilidade $f$-invariantes, $\mu_{1}$ e $\mu_{2} \operatorname{com} \rho\left(\mu_{1}\right)<\rho\left(\mu_{2}\right)$, então para qualquer racional $p / q$ tal que $\rho\left(\mu_{1}\right)<p / q<\rho\left(\mu_{2}\right)$, existe uma órbita $q$-periódica de $f$ com esse número de rotação (ou o que é o mesmo, se $p / q \in \rho(\widetilde{f}$ ), existe $\widetilde{z} \in \widetilde{A}$ tal que $\widetilde{f}^{q}(\widetilde{z})=\widetilde{z}+(p, 0)$ o qual implica que $f$ tem uma órbita $q$-periódica).

Então, suponha $f$ um homeomorfismo irrotacional, e que exista uma medida com número de rotação positivo. Pela versão do teorema de Conley-Zehnder para o anel ([8]), existem pontos fixos com número de rotação nulo. A questão é a seguinte: É verdade que na situação anterior existem órbitas com número de rotação negativo?.

A conjectura anterior ainda é um problema aberto. Nesta dissertação, começaremos trabalhando com os homeomorfismos $f$, que preservam orientação e as componentes de fronteira, com numero de rotação nas fronteiras estritamente positivo, e que tem um levantamento $\tilde{f}$ transitivo, isto é $\widetilde{f}$ tem uma órbita densa (notemos que se $\widetilde{f}$ é transitivo implica que $f$ o é, mas o recíproco nem sempre é verdadeiro).

O motivo para trabalhar com os homemorfismos que tem um levantamento transitivo é que por ([3]), a transitividade do levantamento é satisfeita para um conjunto residual dos homeomorfismos irrotacionais. Neste contexto, daremos certas definições e provaremos alguns resultados que permitem assegurar que o zero está no interior do conjunto de rotação, provando assim a conjectura para os homeomorfismos irrotacionais com levantamento transitivo e sem pontos fixos na fronteira. Além disso, daremos alguns resultados interessantes acerca do comportamento de um conjunto particular que definiremos (o qual serve para provar o resultado anterior) e suas componentes conexas.

No final do trabalho, definiremos um conjunto maior dos homeomorfismos do anel, e trabalharemos para provar que o zero está no interior do conjunto de rotação destes homeomorfismos, obtendo outro resultados interessantes. 


\section{Capítulo 2}

\section{Apresentação dos resultados principais}

\subsection{Notações, definições e resultados principais}

Notação: Dizemos que $A=S^{1} \times[0,1]$ é o Anel, e chamaremos de faixa o conjunto $\widetilde{A}=R \times[0,1]$, o recobrimento universal do anel. Denotaremos por $p: \widetilde{A} \mapsto A$ a aplicação de recobrimento da faixa infinita sobre o anel, por $p_{1}: \widetilde{A} \rightarrow R$ a projeção na primeira coordenada e por $f: A \longrightarrow A$ um homeomorfismo do anel que preserva as componentes de fronteira, isto é, que manda pontos da fronteira en pontos da mesma componente conexa da fronteira.

Definição: Dizemos que $\widetilde{f}: \widetilde{A} \longrightarrow \widetilde{A}$ é um levantamento de $f$ se satisfaz que

$$
p \circ \tilde{f}=f \circ p
$$

Notar aqui, que $f$ tem mais de um levantamento.

Definição: Dizemos que $f$ preserva orientação, se o levantamento $\widetilde{f}$ satisfaz

$$
\widetilde{f}(\widetilde{x}+1, \widetilde{y})=\widetilde{f}(\widetilde{x}, \widetilde{y})+(1,0) \quad \forall(\widetilde{x}, \widetilde{y}) \in R \times[0,1]
$$

Definição: Seja $\widetilde{f}: \widetilde{A} \rightarrow \widetilde{A}$ um homeomorfismo, dizemos que $\widetilde{f}$ é transitivo, se dados quaisquer dois abertos $U, V$ em $\widetilde{A}$ existe $n$ inteiro positivo tal que $\widetilde{f}^{n}(U) \cap V \neq \emptyset$.

De ([2]), temos que se $\tilde{f}$ é transitiva, então $\tilde{f}$ tem uma órbita densa.

Definição: Se $f: A \rightarrow A$ é um homeomorfismo, dizemos que $x_{0}$ é um ponto não errante, se para todo $U \in A, U$ contendo $x_{0}$, existe um inteiro positivo $n_{0}$ tal que $f^{n_{0}}(U) \cap U \neq \emptyset$.

Definição: Seja $\widetilde{f}: \widetilde{A} \rightarrow \widetilde{A}$ um homeomorfismo que preserva orientação e as componentes de fronteira, definimos em $\widetilde{A}$ o seguinte conjunto

$$
B=\bigcap_{n \leq 0} \tilde{f}^{n}((-\infty, 0] \times[0,1])
$$

Notemos que, pelo fato de $(-\infty, 0] \times[0,1]$ ser fechado e $\widetilde{f}$ um homeomorfismo, $B$ é um conjunto fechado.

Para apresentar os resultados principais, daremos a seguinte definição

Definição: $B^{-}$é o seguinte conjunto:

$$
B^{-}=\bigcup_{i \in I} \Gamma_{i}
$$


onde $\Gamma_{i}$ é uma componente conexa não limitada do conjunto $B$, e a união é sobre todas as componentes conexas não limitadas do conjunto $B$.

No trabalho, na verdade, construiremos o $B^{-}$e da construção sairá que ele tem essa forma. Agora estamos em condições de apresentar os resultados principais.

Na primeira parte, vamos considerar os seguintes homeomorfismos:

Definição: Sejam os homeomorfismos $f: A \rightarrow A$ que preservam a orientação, as componentes de fronteira, e que, além disso, possuem um levantamento transitivo. Tais homeomorfismos serão denotados por $\mathrm{Hom}_{+}^{\text {trans }}(A)$.

Estes homeomorfismos serão estudados no capítulo 4, e os resultados principais (os quais aparecem em [5]) são os seguintes

Teorema 1 Se $f \in \operatorname{Hom}_{+}^{\text {trans }}(A)$ e o número de rotação de $(f, \widetilde{f})$ restrito nas fronteiras do anel, $\rho\left(\left.\tilde{f}\right|_{R \times\{i\}}\right)$ é estritamente positivo para $i=0,1$, então o conjunto $B^{-}$é fechado e não vazio $e$ satisfaz que

$$
B^{-} \cap\{0\} \times[0,1] \neq \emptyset .
$$

Também estudaremos o conjunto $\omega$-limite de $B^{-}$e provaremos que:

Teorema 2 Nas mesmas hipóteses do Teorema 1, temos que:

$$
\omega\left(B^{-}\right)=\bigcap_{n=0}^{\infty} \overline{\left(\bigcup_{i=n}^{\infty} \tilde{f}^{i}\left(B^{-}\right)\right)}=\emptyset .
$$

Ou seja, as iteradas de $B^{-}$pela $\widetilde{f}$ convergem para o fim esquerdo de $\widetilde{A}$. As propriedades de $B^{-}$ podem-se estender para provar o seguinte resultado:

Teorema 3 Existe um número real $\rho^{+}\left(B^{-}\right)<0$ tal que, se $\widetilde{z} \in B^{-}$, então

$$
\limsup _{n \rightarrow \infty} \frac{p_{1}\left(\widetilde{f}^{n}(\widetilde{z})\right)-p_{1}(\widetilde{z})}{n} \leq \rho^{+}\left(B^{-}\right)<0
$$

Fazendo uma interpretação geométrica dos resultados anteriores, podemos falar que existe um conjunto na faixa infinita, chamado $B^{-}$cujos pontos convergem para a esquerda de tal faixa, com uma velocidade minima estritamente negativa, e sempre no mesmo conjunto. Note que não falamos nada acerca da velocidade particular (número de rotação) de cada ponto em $B^{-}$, ou seja, pode acontecer que $\rho(x, y)$ não possa ser calculado para nenhum ponto em $B^{-}$, mas, caso exista, $\rho(x, y)$ tem que ser negativo.

Outra conseqüência interessante do Teorema 3 é que, embora não possa garantir a existência de algum ponto com número de rotação no intervalo $\left(\rho^{+} ; 0\right)$, caso exista, este ponto não estaría no conjunto $B^{-}$. Logo o Teorema 3 , somado ao resultado em ([1]) implica que:

Corolário 1 Seja $f \in \operatorname{Hom}_{+}^{\text {trans }}(A)$. Se $\widetilde{f}$ não tem pontos fixos na fronteira de $\widetilde{A}$, então o conjunto de rotação é um intervalo com o 0 no seu interior.

Este corolário mostra que a conjectura apresentada no capítulo anterior é verdadeira para homeomorfismos irrotacionais do anel, sem pontos fixos na fronteira e com um levantamento transitivo.

No desenvolvimento do trabalho, vamos definir um conjunto $D$ genérico, com certas características, as quais são satisfeitas pelos conjuntos $B^{-}$e $\omega\left(B^{-}\right)$, o omega limite de $B^{-}$(caso ele não seja vazio), e o estudo da projeção deste conjunto sobre o anel, ajuda a provar alguns dos resultados 
anteriores. Mas agora, estamos interessados em estudar o próprio conjunto $D$, e a dinâmica dos homeomorfismos definidos antes, quando agem sobre tal conjunto.

Este é o objetivo do capítulo 5, mas agora vamos apresentar o conjunto $D$ e os resultados que serão provados nesse capítulo (este conjunto e o trabalho do capítulo 5 aparece em [7]).

Definição: $D$ é um conjunto não vazio, fechado em $\widetilde{A}$ que satisfaz as seguintes propriedades:

- $\widetilde{f}(D) \subset D$.

- $D \subset(-\infty, 0) \times[0,1]$.

- Toda componente conexa de $D$ é ilimitada a esquerda.

- $D \cap \mathbf{R} \times\{i\}=\emptyset, i \in\{0,1\}$.

- $\widetilde{z} \in D$ então $\widetilde{z}-(1,0) \in D$.

Os resultados principais a provar neste capítulo são:

Teorema $4: p(D)$ é denso em $A$.

Teorema 5 : Existe uma componente conexa $\Gamma$ de $D$, tal que $p(\Gamma)$ é densa em $A, \widetilde{f}(\Gamma) \subset \Gamma$, e existe um inteiro positivo $k$ tal que $\widetilde{f}^{-1}(\Gamma) \subset \Gamma+(k, 0)$, ou seja $f(p(\Gamma))=p(\Gamma)$.

Teorema 6 : Se $\Gamma$ é uma componente conexa de $D$, então $\Gamma-(1,0) \subset \Gamma$.

No último capítulo, vamos definir o seguinte conjunto (tal trabalho foi feito a partir de [6]).

Definição: Seja $H_{+}^{\text {inst }}$ o conjunto dos homeomorfismos que preservam orientação e as componentes de fronteira, e tais que, para qualquer vizinhança $U$ de $\mathbf{S}^{1} \times\{0\}$ e qualquer vizinhança $V$ de $\mathbf{S}^{1} \times\{1\}$, existem inteiros positivos $k_{1}$ e $k_{2}$ tais que $f^{k_{1}}(U) \cap V \neq \emptyset$ e $f^{k_{2}}(V) \cap U \neq \emptyset$.

E vamos pedir que tais homeomorfismos satisfaçam também a seguinte definição

Definição: Dizemos que o homeomorfismo $f$ tem um levantamento não limitado a esquerda $\widetilde{f}$, se para qualquer $M>0$, existe um ponto $\widetilde{z} \in \widetilde{A}$ e um inteiro positivo $n$ tal que $p_{1}\left(\widetilde{f}^{n}(\widetilde{z})\right)-p_{1}(\widetilde{z})<-M$.

No transcurso do trabalho, mostraremos que, se $f \in H_{o m}^{\text {trans }}$, então $f$ satisfaz estas propriedades, logo os resultados principais são os seguintes

Teorema $7:$ Seja $f \in H_{+}^{\text {inst }}$, tal que $\tilde{f}$ é não limitada à esquerda e o número de rotação de ambas fronteiras é estritamente positivo. Então 0 está no interior de $\rho(\widetilde{f})$.

Este resultado, basicamente mostra que, dado que $\rho(\widetilde{f})$ é um intervalo fechado, e como o fato de $\widetilde{f}$ preservar as componentes de fronteira e a orientação implica que ele é homotópico à identidade, ([1]) assegura que existem pontos com número de rotação negativo. Mais ainda, como 0 está no interior de $\rho(\widetilde{f})$, existem pontos $\widetilde{z} \in \widetilde{A}$ que satisfazem que

$$
\limsup _{n \rightarrow \infty} \frac{p_{1}\left(\widetilde{f}^{n}(\widetilde{z})\right)-p_{1}(\widetilde{z})}{n}<0
$$

Um dos resultados deste trabalho também é analisar quais propriedades dinámicas dos homeomorfismos estão implicadas pelos extremos dos intervalos de rotação. Um dos resultados apresentados analisa quando a seguinte propriedade

$$
\inf _{\widetilde{z} \in \widetilde{A}, n \in \widetilde{A}}\left\{p_{1}\left(\widetilde{f}^{n}(\widetilde{z})\right)-p_{1}(\widetilde{z})\right\}-a n>-\infty
$$


é satisfeita. Agora, do teorema anterior, o seguinte resultado sai como conseqüência direta:

Corolário $2:$ Seja $f \in H_{+}^{\text {inst }}$, tal que $\rho(\widetilde{f})=[a, b]$, onde a é racional, e tal que o número de rotação de ambas fronteiras é estritamente maior que a. Então existe um número real $M>0$ tal que, para cada $\widetilde{z} \in \widetilde{A}$, e qualquer inteiro positivo $n$ se tem que

$$
p_{1}\left(\widetilde{f}^{n}(\widetilde{z})\right)-p_{1}(\widetilde{z})-a n>-M
$$

Note que este corolário é o análogo ao resultado 1.2 para os homeorfismos do anel, mostrando como a projeção na primeira coordenada da traslação de um ponto $\widetilde{z}$ atraves das iteradas positivas de $\tilde{f}$ tem um afastamento limitado a esquerda em relação ao extremo inferior do intervalo de rotação.

Um objetivo interessante neste trablho seria poder assegurar alguma coisa acerca do tamanho do conjunto de pontos como velocidade negativa. Para obter algum resultado nessa direção, vamos começar definindo novamente os conjuntos $B^{-}$e $\omega\left(B^{-}\right)$, e um conjunto novo, que será chamado $K_{c}$, o qual vai conter pontos com velocidade negativa; feito isso, geralizaremos alguns resultados sobre os conjuntos $B^{-}$e $\omega\left(B^{-}\right)$, para poder apresentar alguns resultados importantes acerca do conjunto $K_{c}$.

Começamos então com a seguinte

Definição: Seja $f \in H_{+}^{\text {inst }}$, tal que o levantamento $\tilde{f}$ é não limitado à esquerda e o número de rotação de ambas fronteiras é estritamente positivo. Temos então que $\rho(\widetilde{f})=[a, b]$, com $a<0<b$, e para qualquer número real positivo $c$ definimos o conjunto:

$$
K_{c}=\overline{\left\{x \in A, \limsup _{n \rightarrow \infty} \frac{p_{1}\left(\widetilde{f}^{n}(\widetilde{z})\right)-p_{1}(\widetilde{z})}{n}<c+a\right\}}
$$

onde $\widetilde{z} \in \widetilde{A}$ é qualquer ponto que satisfaz que $p(\widetilde{z})=x$.

Aqui notemos que, pela definição do limite superior, o conjunto $K_{c}$ é invariante, pois se um ponto $x \in K_{c}$ então a sua órbita toda está em $K_{c}$.

Logo, temos o seguinte resultado:

Teorema 8 : Para qualquer $\epsilon>0$, qualquer componente conexa de $K_{\epsilon}^{C}$ (o complemento de $K_{\epsilon}$ ) é homotopicamente trivial.

Notemos primeiro aqui que $K_{\epsilon}^{C}$ é um conjunto aberto, logo o fato de que qualquer componente conexa deste conjunto é homotópicamente trivial, implica que estes conjuntos abertos em $A$ não possuem uma curva que seja homotopicamente não trivial, isto é, uma curva fechada simples que de uma volta completa ao anel.

Definição: Dizemos que o ponto $x \in A$ é um ponto recorrente para $f$ se existe uma subseqüência $\left\{n_{k}\right\} \subset \mathbf{N}$ tal que $\lim _{k \rightarrow \infty} f^{n_{k}}(x)=x$. E denotamos por $R_{f}$ o conjunto de todos os pontos recorrentes de $f$.

Uma observação para fazer aqui é que se $x \in A$ é um ponto recorrente, então ele é um ponto não errante.

Logo temos os seguintes corolários:

Corolário 3 : Para qualquer $\epsilon>0$, se $x \in R_{f} \cap K_{\epsilon}^{C}$ e $x$ possui um numero de rotação, então o número de rotação é racional. Se, adicionalmente, $f$ for transitiva no anel, então, ou $K_{\epsilon}=A$ ou $K_{\epsilon}$ será um conjunto cujo interior é vazio e existirá um único racional $p / q$ tal que, se $x \in R_{f} \cap K_{\epsilon}^{C}$ e ele possui número de rotação, ele é $p / q$.

Se além da transitividade, asumimos que $f$ preserva a medida de Lebesgue, então: 
Corolário 4 : Para qualquer $\epsilon>0$, toda componente conexa de $K_{\epsilon}^{C}$ é uma ilha periódica, isto é, um conjunto aberto, homotopicamente trivial, invariante através de alguma iterada de $f$. 


\section{Capítulo 3}

\section{Preliminares.}

Neste capítulo mostraremos alguns resultados que serão de utilidade ao longo do trabalho.

\section{$3.1 \quad$ Ferramentas básicas}

Para começar, vamos lembrar a compactificação L-R da faixa $\widetilde{A}$ como a topologia produto $\tau$. Definimos a compactificação L-R de maneira a obter um disco $\widehat{A}=\widetilde{A} \cup\{+\infty\} \cup\{-\infty\}$ compacto, com a topologia $\widehat{\tau}$ que contém

$$
\widehat{\tau} \supset \tau \cup\{(-\infty ; a) \times[0,1] \cup\{-\infty\}\} \cup\{(b ;+\infty) \times[0,1] \cup\{+\infty\}\}
$$

Notemos que com esta topologia, $\widehat{A}$ é, basicamente, um disco Hausdorff e compacto. Além disso, notemos que, se chamamos $-\infty=L$ e $+\infty=R, \widetilde{f}$ induz um homeomorfismo $\widehat{f}$ sobre $\widehat{A}$ que satisfaz $\widehat{f}(L)=L, \widehat{f}(R)=R$ e $\widehat{f}(x)=\widetilde{f}(x)$, para todo $x \in \widetilde{A}$.

Uma consequência importante na definição da topologia $\widehat{\tau}$, é que, os abertos contendo $L$ (respectivamente $R$ ), são os conjuntos que contém uma semi-faixa do tipo

$$
\{L\} \cup\{(-\infty, a) \times[0,1]\}(\{L\} \cup\{(b, \infty) \times[0,1]\})
$$

para algum $a$ (ou $b$ ) em R.

Um resultado preliminar que vamos precisar posteriormente, tem a ver com a noção de distância e convergência num subconjunto das partes de $R^{n}$. É por isso que vamos definir seu significado.

Vamos começar definindo uma distância nas parte compactas de $R^{n}$ (no nosso caso, é suficiente provar para $R^{2}$, ou num disco fechado desse espaço, que será compacto com a topologia herdada). Logo a idéia é a seguinte, definida a distância nas partes compactas de $R^{n}$, vamos definir seqüência de Cauchy de compactos, convergencia de compactos num compacto, e a convergencia de uma seqüência de compactos, o passo final será provar que, num disco fechado de $R^{2}$, o conjunto definido pela seqüência convergente de compactos é fechado, logo será compacto no disco, como uma propriedade particular também mostraremos que se a seqüência de compactos é conexa, o conjunto limite também o é.

Seja $d: \mathbf{R}^{n} \times \mathbf{R}^{n} \rightarrow \mathbf{R}$ a distância usual, e sua topologia métrica associada. Lembremos que dado um ponto $x_{1} \in \mathbf{R}^{n}$ e um compacto $K_{2} \subset \mathbf{R}^{n}$, temos bem definida a distância do ponto ao compacto, que é dada por

$$
d\left(x_{1}, K_{2}\right)=\inf _{y \in K_{2}} d\left(x_{1}, y\right)
$$

e esta distância é, de fato, o mínimo (pois a função $d$ é contínua e $K_{2}$ é fechado e limitado), e é atingida num $y_{1} \in K_{2}, \log 0$

$$
d\left(x_{1}, K_{2}\right)=\inf _{y \in K_{2}} d\left(x_{1}, K_{2}\right)=\min _{y \in K_{2}} d\left(x_{1}, K_{2}\right)=d\left(x_{1}, y_{1}\right)
$$


Agora, se $x_{1} \in K_{1}$ com $K_{1}$ compacto em $\mathbf{R}^{n}$ podemos definir (pelos mesmos motivos que acima)

$$
\sup _{x_{1} \in K_{1}} d\left(x_{1}, K_{2}\right)=\max _{x_{1} \in K_{1}} d\left(x_{1}, K_{2}\right)
$$

Agora vamos definir $\widetilde{d}: P_{C}\left(\mathbf{R}^{n}\right) \times P_{C}\left(\mathbf{R}^{n}\right) \rightarrow \mathbf{R}$ (onde $P_{C}\left(\mathbf{R}^{n}\right)$ são as parte compactas de $\mathbf{R}^{n}$ ) do seguinte modo

$$
\widetilde{d}\left(K_{1}, K_{2}\right)=\max _{x_{1} \in K_{1}} d\left(x_{1}, K_{2}\right)+\max _{x_{2} \in K_{2}} d\left(x_{2}, K_{1}\right)
$$

Vejamos que $\widetilde{d}$ define precisamente uma distância, isto é, que satisfaz as seguintes propriedades:

- $\widetilde{d}\left(K_{1}, K_{2}\right) \geq 0 \quad \forall K_{1}, K_{2} \in P_{C}\left(\mathbf{R}^{n}\right)$.

Isto é claro, dado que $d\left(x_{1}, K_{2}\right)$ e $d\left(x_{2}, K_{1}\right)$ são ambas maiores que 0 , logo, seu máximo também o é.

- $\widetilde{d}\left(K_{1}, K_{2}\right)=0 \Leftrightarrow K_{1} \equiv K_{2}$

Se $K_{1} \equiv K_{2}$ então é claro que $\widetilde{d}\left(K_{1}, K_{2}\right)=0$ Para a outra implicação, o objetivo é provar que ambos conjuntos são coincidentes, mas se $\widetilde{d}\left(K_{1}, K_{2}\right)=0$ então, pela propriedade anterior temos que

$$
0=\max _{x_{1} \in K_{1}} d\left(x_{1}, K_{2}\right)=\max _{x_{2} \in K_{2}} d\left(x_{2}, K_{1}\right)
$$

Logo, por exemplo, $d\left(x_{1}, K_{2}\right)=0$, e, pelo fato de $K_{2}$ ser compacto e $d$ contínua, temos que $x_{1} \in K_{2}$ para todo $x_{1} \in K_{1}$, logo $K_{1} \subseteq K_{2}$, da mesma forma, $K_{2} \subseteq K_{1}$, e temos a outra implicação.

- $\widetilde{d}\left(K_{1}, K_{2}\right)=\widetilde{d}\left(K_{2}, K_{1}\right)$

Trivial

- $\widetilde{d}\left(K_{1}, K_{2}\right) \leq \widetilde{d}\left(K_{1}, M\right)+\widetilde{d}\left(M, K_{2}\right) \forall M \in P_{C}\left(\mathbf{R}^{n}\right)$

Pela definição de $\widetilde{d}$ é claro que

$$
\widetilde{d}\left(K_{1}, M\right)+\widetilde{d}\left(M, K_{2}\right) \geq\left(d\left(\widetilde{x}_{1}, M\right)+d\left(\widetilde{m}, K_{1}\right)\right)+\left(d\left(\widehat{m}, K_{2}\right)+d\left(\widetilde{x}_{2}, M\right)\right)
$$

e isso acontece para todo $\widetilde{x}_{1} \in K_{1}$, para todo $\widetilde{x}_{2} \in K_{2}$, e para todo $\widetilde{m}, \widehat{m} \in M$, logo, em particular, isso acontece para $m_{0}$ e $m_{1}$, sendo estes, os os pontos de $M$ onde as funções $d\left(\widetilde{x}_{1}, M\right)$ e $d\left(\widetilde{x}_{2}, M\right)$ atingem seu mínimo respectivamente, então temos que o lado direito da desigualdade anterior e igual a

$$
d\left(\widetilde{x}_{1}, m_{0}\right)+d\left(\widetilde{m}, K_{1}\right)+d\left(\widehat{m}, K_{2}\right)+d\left(\widetilde{x}_{2}, m_{1}\right)
$$

Logo, como o anterior é valido para todo $\widetilde{m}, \widehat{m} \in M$ temos que o anterior é igual a

$$
d\left(\widetilde{x}_{1}, m_{0}\right)+d\left(m_{0}, K_{2}\right)+d\left(m_{1}, K_{2}\right)+d\left(\widetilde{x}_{2}, m_{1}\right)
$$

mas por propriedade da função $d$ temos que

$$
d\left(\widetilde{x}_{1}, m_{0}\right)+d\left(m_{0}, K_{2}\right)+d\left(m_{1}, K_{2}\right)+d\left(\widetilde{x}_{2}, m_{1}\right) \geq d\left(\widetilde{x}_{1}, K_{2}\right)+d\left(\widetilde{x}_{2}, K_{1}\right)
$$

e como o de acima acontece para todo $\widetilde{x}_{1} \in K_{1}$, e para todo $\widetilde{x}_{2} \in K_{2}$, temos, tomando o 
máximo em $K_{1}$ e o máximos em $K_{2}$ que

$$
\widetilde{d}\left(K_{1}, K_{2}\right) \leq \widetilde{d}\left(K_{1}, M\right)+\widetilde{d}\left(M, K_{2}\right)
$$

$\forall M \in P_{C}\left(\mathbf{R}^{n}\right)$

Portanto, provamos que $\widetilde{d}$ é uma distância em $P_{C}\left(\mathbf{R}^{n}\right)$. Agora podemos definir o que é uma seqüência de Cauchy em $P_{C}\left(\mathbf{R}^{n}\right)$.

Definição: Dizemos que a seqüência $\left\{K_{n}\right\}_{n=1}^{\infty}$ de compactos em $\mathbf{R}^{n}$ é uma seqüência de cauchy se satisfaz que dado $\epsilon>0$, existe $n_{0}$ inteiro positivo tal que se $n, m \geq n_{0}$ então $\widetilde{d}\left(K_{n}, K_{m}\right)<\epsilon$.

A idéia é a seguinte, vamos definir a convergência de uma seqüência de Cauchy de compactos sobre um conjunto compacto, logo, vamos definir, em geral, o conjunto limite de uma seqüência de Cauchy de compactos, e vamos provar que é um conjunto compacto, provando logo que as duas definições coincidem (isto é que se a seqüência de compactos converge a um compacto $K$, este último é, precisamente o conjunto limite).

Definição Seja $\left\{K_{n}\right\}_{n=1}^{\infty}$ como antes, e $K \subset \mathbf{R}^{n}$ compacto, dizemos que a seqüência $\left\{K_{n}\right\}_{n=1}^{\infty}$ converge a $K$, e o denotamos por $K_{n} \rightarrow K$, se dado $\epsilon>0$, existe $n_{0}$ inteiro positivo tal que se $n \geq n_{0}$ então $\widetilde{d}\left(K_{n}, K\right)<\epsilon$.

Uma observação: note que os mesmos resultados valem tanto em $R^{n}$ como em $\widehat{A}$, com uma diferença importante, como $\widehat{A}$ é compacto, a topologia induzida por $\widetilde{d}$ (topologia métrica) sobre as partes compactas eu posso assegurar que qualquer seqüência de compactos possui uma subseqüência convergente .

Definição: Seja $\left\{K_{n}\right\}_{n=1}^{\infty}$ uma seqüência de Cauchy de conjuntos compactos definimos o conjunto limite $\widetilde{K}=\left\{x \in \mathbf{R}^{n}\right.$ satisfazendo que existe $x_{i} \in K_{i} \mathrm{i}=1,2 \ldots$ e $\left.x_{i} \rightarrow x\right\}$, e o denotamos por

$$
\widetilde{K}=\lim _{n \rightarrow \infty} K_{n}
$$

Ou seja $\widetilde{K}$ é o conjunto de todos os limites de todas as seqüências de Cauchy $\left\{x_{n}\right\} \operatorname{com} x_{i} \in K_{i}$.

Proposição 3.1.1 : Seja $\left\{K_{n}\right\}_{n=1}^{\infty}$ uma seqüência de Cauchy de conjuntos compactos. O conjunto

$$
\widetilde{K}=\lim _{n \rightarrow \infty} K_{n}
$$

é um conjunto compacto em $\mathbf{R}^{n}$.

Demonstração: Só é preciso mostrar que o conjunto é fechado e limitado em $R^{n}$. Que é limitado, sai do fato de $\left\{K_{n}\right\}$ ser uma seqüência de cauchy de conjuntos compactos e da definição de $\widetilde{K}$.

Para ver que é fechado, deve-se provar que $\widetilde{K}$ contém todos seus pontos de acumulação. Então, seja $\widetilde{z} \in \mathbf{R}^{n}$ tal que existe $\left\{z_{i}\right\}_{i \in \mathbf{N}} \subset \widetilde{K}$, e tal que $z_{i} \rightarrow \widetilde{z}$, vamos provar que $\widetilde{z} \in \widetilde{K}$.

Dado que $z_{i} \in K$, para cada i, existe uma seqüência de cauchy $\left\{z_{i}^{j}\right\}$ nas condições de acima. Então, dado $\epsilon_{1}=1$, existe $i_{0} \in \mathbf{N}$ tal que se $i \geq i_{0}$ temos que $d\left(z_{i}, \widetilde{z}\right)<\frac{1}{4}$. E também para este mesmo $\epsilon_{1}$, existe $\tilde{i_{0}}$, tal que se $l, r \geq \widetilde{i_{0}}$, então $d\left(z_{l}, z_{r}\right)<\frac{1}{2}$.

Escolhemos $i_{1}=\max \left\{i_{0}, \tilde{i_{0}}\right\}$, e tomamos $z_{i_{1}}$. Notemos que por nossa escolha, existe $j_{1}$ suficientemente grande tal que da seqüência $\left\{z_{i}^{j}\right\}$ convergendo a $z_{i_{1}}, z_{i_{1}}^{j_{1}}$ satisfaz que $d\left(z_{i_{1}}^{j_{1}}, z_{i_{1}}\right)<\frac{1}{2}$, $\log 0$

$$
d\left(z_{i_{1}}^{j_{1}}, \widetilde{z}\right)<1=\epsilon_{1}
$$

chamemos então

$$
x_{j_{1}}=z_{i_{1}}^{j_{1}}
$$


logo podemos escrever

$$
d\left(x_{j_{1}}, \widetilde{z}\right)<1=\epsilon_{1}
$$

Em geral, dado $\epsilon_{n}=\frac{1}{2^{n-1}}$, fazendo o mesmo proceso, vamos achar $i_{n}=\max \left\{i_{n-1}, \widetilde{i}_{n-1}\right\}$. Tomando $z_{i_{n}}$ e $j_{n}$ suficientemente grandes, $j_{n}>j_{n-1}$, eu obtenho, chamando

$$
x_{j_{n}}=z_{i_{n}}^{j_{n}}
$$

que

$$
d\left(x_{j_{n}}, \widetilde{z}\right)<\frac{1}{2^{n-1}}=\epsilon_{n}
$$

Para conluir, devemos obter uma seqüência convergente a $\widetilde{z}$ nas condições da definição, isto é cada $x_{i}$ tem que estar em $K_{i}$ para $i \in \mathbf{N}$, mas isso é obtido completando a seqüência da seguinte forma, dado que $j_{n+1}>j_{n}$ para todo inteiro positivo $n$, definimos

$$
x_{l}=z_{i_{n}}^{l}, \quad j_{n} \leq l<j_{n+1} \quad \text { e } \quad x_{l}=x_{j_{n+1}}, \quad l=j_{n+1}
$$

Logo por ser cada seqüência uma seqüência de cauchy, e por construção, a seqüência $\left\{x_{n}\right\}_{n \in \mathbf{N}}$ é uma sequiência de cauchy convergindo a $\widetilde{z}$ e tal que $x_{i} \in K_{i}$ para todo $i$, portanto $\widetilde{z} \in \widetilde{K}$ por definição, obtendo assim que $\widetilde{K}$ é compacto.

Agora, o que falta provar é que se eu tenho $K_{n} \rightarrow K$, segundo a definição, então $K$ é precisamente o conjunto limite, mas isso decorre do seguinte resultado.

Proposição 3.1.2 Seja $\left\{K_{n}\right\}_{n=1}^{\infty}$ uma seqüência de compactos, $K$ compacto, $K_{n} \rightarrow K, x_{i} \in K_{i}$, $i \in \mathbf{N}$, tal que $x_{i} \rightarrow x$, então $x \in K$

Demonstração: Notemos que como $K$ é compacto, provar a proposição é provar que $d(x, K)=0$. Então, dado $\epsilon>0$, existe $n_{1}$ inteiro positivo, satisfazendo que, se $n \geq n_{1}$ temos $\widetilde{d}\left(K_{n}, K\right)<\frac{\epsilon}{2}$. E também temos que existe $n_{2}$ inteiro positivo, tal que se $n \geq n_{2}$ então $d\left(x_{n}, x\right)<\frac{\epsilon}{2}$.

Tomando $n_{0}=\max \left\{n_{1}, n_{2}\right\}$, obtemos que $d(x, K)<\frac{\epsilon}{2}$. Logo, como isso vale para todo $\epsilon>0 \mathrm{e}$ $K$ é fechado, temos o pedido.

Temos então que as duas definições coincidem, logo vamos usar indistintamente uma notação o a outra.

Proposição 3.1.3 : Seja $\left\{K_{n}\right\}$ é uma seqüência de compactos e conexos que converge a $K$, então $K$ é conexo.

Demonstração: Caso contrário, $K$ é pelo menos união de dois compactos disjuntos $\widetilde{K}_{1}$ e $\widetilde{K}_{2}$, cuja distância entre eles é $\delta>0$, mas como a seqüência de compactos converge, para um $n$ sficientemente grande, a distância $\widetilde{d}$ entre $K_{n}$ e $\widetilde{K}_{i}$ é menor que $\frac{\delta}{2}$, para $i=1,2$, com o qual obtemos, pela desigualdade triangular, um contradição que prova nossa afirmação.

Antes de definir os conjuntos como os quais vamos trabalhar, vamos provar a seguinte afirmação:

Afirmação 3.1.1 : Se $\widetilde{f}$ é transitiva, então todo ponto de $\widetilde{A}$ é não errante.

Prova: A prova é clara. Dado $x_{0} \in \widetilde{A}$ e $U$ aberto contendo o ponto, temos que, pelo fato de $\widetilde{f}$ ter uma orbita densa, existe $n_{0} \in \mathbf{Z}$ e $z_{0} \in \widetilde{A}$ tal que $\widetilde{f}^{n_{0}}\left(z_{0}\right) \in U$. Agora, tomemos $V \subset U$ aberto, tal que $\widetilde{f}^{n_{0}}\left(z_{0}\right)$ não esteja em $V$, agora como o mesmo $\widetilde{f}^{n_{0}}\left(z_{0}\right)=y_{0}$ tem uma orbita densa, existe $m_{0} \in \mathbf{Z}$ tal que $\widetilde{f}^{m_{0}}\left(y_{0}\right) \in V$, se $m_{0}>0$ temos o pedido, e se $m_{0}<0$ só consideramos $-m_{0}$ e também obtemos nossa afirmação. 
Afirmação 3.1.2 : Se $\Omega_{1}$ e $\Omega_{2}$ são conjuntos abertos e conexos tais que $\Omega_{1} \cap \Omega_{2} \neq \emptyset$ e $\partial \Omega_{2} \cap \Omega_{1}=\emptyset$, então $\Omega_{1} \subset \Omega_{2}$.

Prova: Seja $x \in \Omega_{1} \cap \Omega_{2}$ e seja $y \in \Omega_{1}$ qualquer ponto nesse conjunto, como $\Omega_{1}$ é conexo, existe $\alpha \subset \Omega_{1}$ ligando $x$ e $y$, como $\alpha \cap \Omega_{2}=\emptyset$ temos que $\alpha \subset \partial \Omega_{2}$ obtendo assim o procurado. 


\section{Capítulo 4}

\section{O conjunto $B^{-}$.}

Para começar, vamos lembrar a seguinte definição:

Definição: Seja $\widetilde{f}: \widetilde{A} \rightarrow \widetilde{A}$ um homeomorfismo que preserva orientação e as componentes de fronteira, definimos em $\widetilde{A}$ o seguinte conjunto

$$
B=\bigcap_{n \leq 0} \widetilde{f}^{n}((-\infty, 0] \times[0,1])
$$

Notemos, novamente, pelo fato de $(-\infty, 0] \times[0,1]$ ser fechado e $\widetilde{f}$ um homeomorfismo, $B$ é um conjunto fechado.

\subsection{Demonstração do Teorema 1}

Para começar a demonstração do Teorema 1 vamos introduzir os seguintes conjuntos em $\mathbf{R} \times$ $[0,1]$. Dado um número real $a$, seja:

$$
V_{a}=\{a\} \times[0,1] \quad ; \quad V_{a}^{-}=(-\infty ; a] \times[0,1] \quad ; V_{a}^{+}=[a ;+\infty] \times[0,1]
$$

denotamos por $V, V^{-}$e $V^{+}$os correspondentes a $V_{0}, V_{0}^{-}$e $V_{0}^{+}$e por $\widehat{V}, \widehat{V}^{-}$e $\widehat{V}^{+}$e $\widehat{V}_{a}, \widehat{V}_{a}^{-}$e $\widehat{V}_{a}^{+}$ os correspondentes em $\widehat{A}$.

Notemos que, dado que $\tilde{f}$ é um homeomorfismo, $\widehat{f}$ também o é. Definimos assim

$$
\widehat{B}=\bigcap_{n \leq 0} \widehat{f}^{n}\left(\widehat{V}^{-}\right)
$$

que é fechado. É claro que $L \in \widehat{f}^{n}\left(\widehat{V}^{-}\right)$para todo $n \leq 0$. Logo, como $\widehat{B}$ é não vazio e $\widehat{f}$ é um homeomorfismo temos que

$$
\widehat{f}(\widehat{B})=\widehat{f}\left(\widehat{V}^{-}\right) \cap \bigcap_{n \leq 0} \widehat{f}^{n}\left(\widehat{V}^{-}\right) \subset \widehat{V}^{-} \cap \bigcap_{n \leq-1} \widehat{f}^{n}\left(\widehat{V}^{-}\right)=\widehat{B}
$$

Agora denotemos por $\widehat{B}^{-}$a componente conexa de $\widehat{B}$ que contém $L$ e por $B^{-}$o conjunto correspondente na faixa que, por construção, é a união de todas as componentes conexas ilimitadas de $B$. Ele, em princípio, pode ser vazio. Então o que vamos provar no Lema 4.1.1 é precisamente que o conjunto $B^{-}$é não vazio, mais ainda, vamos provar que intercepta $V$.

Notemos que $B^{-}$é fechado, pois $\widehat{B}^{-}$o é, e pelo falado na seção anterior, $\widehat{B}^{-}$é a união de $\{-\infty\}$ com um fechado, daí obtemos que $B^{-}$é fechado.

E também, no caso de ele ser não vazio, temos que $\widetilde{f}\left(B^{-}\right) \subset B^{-}$, por (4.1).

O que resta para provar do teorema 1 é, precisamente, que o conjunto $\widetilde{B}^{-}$é não vazio e que 
intercepta $V$, mas isso decorre do próximo lema e da afirmação posterior.

Lema 4.1.1 Seja $f: A \rightarrow A$ um homeomorfismo que preserva orientação, e as componentes de fronteira, e fixemos o levantamento $\widetilde{f}: \widetilde{A} \rightarrow \widetilde{A}$. Suponha que para todo a $<0$ existe un inteiro positivo $n$ tal que $\tilde{f}^{n}(V) \cap V_{a}^{-} \neq \emptyset$. Então $\widehat{B}^{-} \cap \widehat{V} \neq \emptyset$ (e o equivalente para a faixa: $B^{-} \cap V \neq \emptyset$ ).

Demonstração: Dado $N>0$, tomamos $a<0$ suficientemente pequeno tal que

$$
n=\inf \left\{i>0: \tilde{f}^{-i}\left(V_{a}\right) \cap V \neq \emptyset\right\}>N
$$

Note que o acima é certo, pois pela continuidade de $\widetilde{f}$, se $|a|$ é muito grande, as iteradas de $V \widetilde{f}$ demoram mais tempo para atingir $V_{a}$. Agora, pela definição de $n$ e o fato de $\widetilde{f}$ preservar orientação, temos que $\widetilde{f}^{-j}\left(V_{a}\right) \subset V^{-}$, para $j=0,1, \ldots, n-1$ e $\widetilde{f}^{n}\left(V_{a}\right) \cap V \neq \emptyset$. O anterior implica que existe um arco contínuo, não limitado a esquerda $\Gamma_{N} \subset \widetilde{f}^{-n}\left(V_{a}^{-}\right) \cap V^{-}$(pois $\widetilde{f}$ preserva orientação), tal que $\widehat{\Gamma}_{N}$ conecta $L$ a $\widehat{V}$, isto é, com um extremo em $L$ e outro em $\widehat{V}$. Então, para este arco $\Gamma_{N}$, tomando $1 \leq i \leq n$ temos, pelo falado acima que $\widetilde{f}^{i}\left(\Gamma_{N}\right) \subset \widetilde{f}^{-n+i}\left(V_{a}^{-}\right) \cap \widetilde{f}^{i}\left(V^{-}\right) \subseteq \widetilde{f}^{-n+i}\left(V_{a}^{-}\right) \subset V^{-}$, $\operatorname{logo} \Gamma_{N} \subset \widetilde{f}^{-i}\left(V^{-}\right)$para todo $i=0, \ldots, n$. Passando à compactificação, temos $\widehat{\Gamma}_{N} \subset \widehat{f}^{-i}\left(\widehat{V}^{-}\right)$, $i=0, \ldots, n$, portanto

$$
\widehat{\Gamma}_{N} \subset \bigcap_{i=0}^{n} \widehat{f}^{-i}\left(\widehat{V}^{-}\right)
$$

com $\widehat{\Gamma}_{N} \cap \widehat{V} \neq \emptyset$. O anterior implica que, fazendo $N \rightarrow \infty$ temos que $n \rightarrow \infty$, logo, temos uma seqüência de compactos e conexos $\left\{\Gamma_{N}\right\}, N \in \mathbf{N}$, todas com extremos em $L$ e $\widehat{V}$, logo, pelo provado no inicio do capítulo, temos que a seqüência posui uma subseqüência convergente a um compacto e conexo $\widehat{\Gamma} \subset \widehat{A}$, que conecta $L$ com $\widehat{V}$. Pela construção, temos que $\widehat{\Gamma} \subset \widehat{B}^{-}$, obtendo assim o Lema.

Afirmação 4.1.1 : Se $f \in \operatorname{Hom}_{+}^{\text {trans }}(A)$, então as hipóteses do Lema 4.1.1 são satisfeitas para $(f, \widetilde{f})$.

Prova: O único que precisamos provar é que, se $\widetilde{f}$ é transitiva, então para todo $a<0$, existe um inteiro positivo $n$ tal que $\widetilde{f}^{n}(V) \cap V_{a}^{-} \neq \emptyset$, lembrando que $\tilde{f}$ preserva orientação e componentes de fronteira.

Suponha que existe $a<0$, tal que para todo inteiro positivo $n, \widetilde{f}^{n}(V) \cap V_{a}^{-}=\emptyset$, notemos que, como $\widetilde{f}$ preserva orientação, o anterior implica que $\widetilde{f}^{n}\left(\right.$ int $\left.V^{+}\right) \cap V_{a}^{-}=\emptyset$

Como $\widetilde{f}$ é transitivo, possui uma órbita densa, ou seja, existe $x_{0} \in i n t V^{+}$, tal que para algum $j_{0} \in \mathbf{Z}, \widetilde{f}^{j_{0}}\left(x_{0}\right) \in i n t V_{a}^{-}$ou seja, pela continuidade de $\widetilde{f}$, existe $U \subset i n t V^{+}$contendo $x_{0}$ e $\widetilde{f}^{j_{0}}(U) \cap$ int $V_{a}^{-} \neq \emptyset$. Por hipóteses $j_{0}<0$.

Pelo provado no capítulo 2 , temos que todo ponto de $\widetilde{f}$ é não errante, chamando $W_{0}=\widetilde{f}^{j_{0}}(U) \cap$ $\operatorname{int} V_{a}^{-}$, temos que $W_{0}$ é diferente de vazio e é aberto, logo existe $i_{0}>0$ tal que $\widetilde{f}^{i_{0}}\left(W_{0}\right) \cap W_{0} \neq \emptyset$.

Logo temos duas possibilidades: $\left|i_{0}\right|>\left|j_{0}\right|$ ou $\left|j_{0}\right|>\left|i_{0}\right|$ :

- $\left|i_{0}\right|>\left|j_{0}\right|$

Se isso acontece, então temos que $i_{0}+j_{0}>0$, então para $x_{0} \in i n t V^{+}$, consideramos $\tilde{f}^{i_{0}+j_{0}}\left(x_{0}\right)$, sendo que $\widetilde{f}^{j_{0}}\left(x_{0}\right) \in i n t V_{a}^{-}$e, pela escolha de $i_{0}, \widetilde{f}^{i_{0}+j_{0}}\left(x_{0}\right) \in i n t V_{a}^{-}$, o que é uma contradição.

- $\left|j_{0}\right|>\left|i_{0}\right|$

Tomando o $W_{0}$ definido antes, temos que $\widetilde{W}_{1}=\widetilde{f}^{i_{0}}\left(W_{0}\right) \cap W_{0} \neq \emptyset$ (pode-se supor que $i_{0}$ é o primeiro inteiro positivo para o qual isso acontece), e como todo ponto de $\widetilde{A}$ é não errante, temos que existe $i_{1}>0$ tal que $\widetilde{W}_{2}=\widetilde{f}^{i_{1}}\left(W_{1}\right) \cap W_{1} \neq \emptyset$, logo $\widetilde{f}^{i_{1}}\left(W_{1}\right) \cap W_{0} \neq \emptyset$, pois por construção, $\widetilde{W}_{1} \subset W_{0}$ e, pela escolha, $i_{1}>i_{0}$ (pois pode-se tomar $i_{1}$ do mesmo modo que $i_{0}$ ).

Em geral, definimos $i_{n}$ tal que $\widetilde{f}^{i_{n}}\left(\widetilde{W}_{n}\right) \cap \widetilde{W}_{n} \neq \emptyset$, e chamamos $\widetilde{W}_{n+1}=\widetilde{f}^{i_{n}}\left(\widetilde{W}_{n}\right) \cap \widetilde{W}_{n}$, e temos 
que, por construção $\widetilde{W}_{n+1} \subset \widetilde{W}_{n} \subset \ldots \subset W_{0}$, logo existe $z_{n+1} \in \widetilde{W}_{i}$ e também $z_{n+1} \in W_{0}$.

Por ser $\widetilde{f}$ um homeomorfismo, temos que existe $z_{0} \in W_{0}$ tal que $z_{n+1}=\widetilde{f}^{i_{0}+\ldots+i_{n}}\left(z_{0}\right)$. Como $i_{j} \geq i_{0}$ para qualquer $j$, temos que existe um $m_{0}$ inteiro positivo e um $\widetilde{z}_{0} \in W_{0}$ satisfazendo o anterior

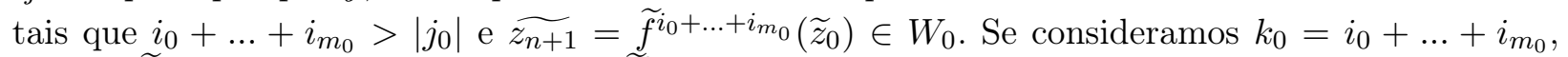
e $y_{0}=\widetilde{f}^{-j_{0}}\left(z_{0}\right) \in i n t V^{+}$Temos que $\widetilde{f}^{k_{0}+j_{0}}\left(y_{0}\right) \in i n t V_{a}^{-}$, o qual é novamente uma contradição. Logo todas as contradições vem de supor que existe $a<0$ tal que para todo inteiro positivo $n$, $\widetilde{f}^{n}(V) \cap V_{a}^{-}=\emptyset$, logo temos que se $\widetilde{f}$ é transitiva, então para todo $a<0$, existe um inteiro positivo $n$ tal que $f^{n}(V) \cap V_{a}^{-} \neq \emptyset$

Logo temos nossa afirmação e portanto a prova do Teorema 1.

De aqui para frente, vamos considerar $f \in \operatorname{Hom}_{+}^{\text {trans }}(A)$ e tal que $\rho\left(\left.\widetilde{f}\right|_{R \times\{i\}}\right)>0$ para $i=0,1$. Note que $B^{-} \subset B \subset V^{-}$e como $\tilde{f}(B) \subset B \subset V^{-}$, e $\rho$ nas fronteiras é positivo pelo resultado 1.2, $B$ e portanto $B^{-}$não intersecta $\mathbf{R} \times\{i\}, i=0,1$. Além disso, $B^{-} \subset \widetilde{A}$ é um conjunto fechado, $B^{-}$ intersecta $V$, limitado a sua direita, cujas componentes conexas são ilimitadas (porque $L \in \widehat{B}^{-}$).

\subsection{O conjunto $\omega\left(B^{-}\right)$}

Aqui, vamos começar analisando o conjunto

$$
\omega\left(\widehat{B}^{-}\right)=\bigcap_{n=0}^{\infty} \overline{\bigcup_{i=n}^{\infty} \widehat{f}^{i}\left(\widehat{B}^{-}\right)}
$$

um subconjunto de $\widehat{A}$ e seu correspondente conjunto na faixa

$$
\omega\left(B^{-}\right)=\bigcap_{n=0}^{\infty} \overline{\bigcup_{i=n}^{\infty} \tilde{f^{i}}\left(B^{-}\right)}
$$

contido em $\widetilde{A}$.

Lema 4.2.1 $\omega\left(B^{-}\right)$é um conjunto fechado, $\tilde{f}$ invariante, cujas componentes conexas são todas ilimitadas.

Demonstração: Vamos estudar primeiro o conjunto $\omega\left(\widehat{B}^{-}\right)$. Notemos que $\widehat{f}\left(\widehat{B}^{-}\right) \subseteq \widehat{B}^{-}$, pois caso contrário, como $\widehat{f}(L)=L$, teríamos que $\widehat{B}^{-} \cap \widehat{f}\left(\widehat{B}^{-}\right) \neq \emptyset$, logo a união dos dois conjuntos sería um conexo que contém $\widehat{B}^{-}$o qual não acontece por definição de $\widehat{B}^{-}$.

Como $\widehat{f}$ é um homeomorfismo e $\widehat{B}^{-}$é fechado, temos que $\widehat{f}^{n}\left(\widehat{B}^{-}\right)$é fechado, compacto (pois fechado num compacto é compacto) e conexo para todo $n$ inteiro positivo. Logo, por todo o anterior

$$
\omega\left(\widehat{B}^{-}\right)=\bigcap_{n=0}^{\infty} \widehat{f}^{n}\left(\widehat{B}^{-}\right)
$$

É uma interseção encaixante de conjuntos conexos e compactos, cuja interseção é não vazia ( $L$ está nessa interseção). Logo, a interseção é conexa e compacta e pela forma como escrevemos o conjunto e o fato de $\widehat{f}$ ser homeomorfismo, é claro que ele é $\widehat{f}$ invariante pois $\widehat{f}^{n}\left(\widehat{B}^{-}\right) \subset \widehat{B}^{-}$para todo $n$. Então

$$
\widehat{f}\left(\omega\left(\widehat{B}^{-}\right)\right)=\widehat{f}\left(\bigcap_{n=0}^{\infty} \widehat{f}^{n}\left(\widehat{B}^{-}\right)\right)=\bigcap_{n=1}^{\infty} \widehat{f}^{n}\left(\widehat{B}^{-}\right)=\bigcap_{n=1}^{\infty} \widehat{f}^{n}\left(\widehat{B}^{-}\right) \cap \widehat{B}^{-}=\omega\left(\widehat{B}^{-}\right)
$$

e temos que $\omega\left(\widehat{B}^{-}\right)$é $\widehat{f}$ invariante.

Agora, todo o anterior vale para $\omega\left(B^{-}\right)$, e como $L \in \omega\left(\widehat{B}^{-}\right)$, temos, por ser ele conexo, que cada componente conexa de $\omega\left(B^{-}\right)$é ilimitada à esquerda. Assim, fica provado o lema. 
Dado que $B^{-}$é fechado, temos que $\omega\left(B^{-}\right) \subset B^{-}$e portanto, o conjunto $\omega$-limite de $B^{-}$não intersecta as fronteiras de $\widetilde{A}$, então a idéia é provar que $\omega\left(B^{-}\right)$é vazio. Então definimos

Definição: $D$ é um conjunto não vazio, fechado em $\widetilde{A}$ que satisfaz as seguintes propriedades:

- $\tilde{f}(D) \subset D$.

- $D \subset(-\infty, 0) \times[0,1]$.

- Toda componente conexa de $D$ é ilimitada a esquerda.

- $D \cap \mathbf{R} \times\{i\}=\emptyset, i \in\{0,1\}$.

- $\widetilde{z} \in D$ então $\widetilde{z}-(1,0) \in D$.

Notemos que os primeiros quatro items são satisfeitos por $B^{-}$e $\omega\left(B^{-}\right)$. Para o último item, temos que, dado $\widetilde{z} \in B^{-}$, se $\Gamma$ é a componente conexa de $B^{-}$que contém $\widetilde{z}, \Gamma$ é conexa, contida em $B^{-}$ e ilimitada a esquerda, além disso, $\Gamma \subset \widetilde{f}^{n}\left(V^{-}\right)$para todo $n \leq 0$, logo, pelo fato de $\tilde{f}$ preservar orientação temos que $\Gamma-(1,0) \subset \widetilde{f}^{n}\left(V^{-}-(1,0)\right) \subset \widetilde{f}^{n}\left(V^{-}\right)$para qualquer $n \leq 0$, por tanto, $\Gamma-(1,0) \subset B$, é conexo e ilimitado a esquerda, logo ele está em $B^{-}$. A idéia para o omega limite é a mesma, só trabalhar com a definição de $\omega\left(B^{-}\right)$.

Notemos uma conseqüência importante da última propriedade, o fato de que $\widetilde{B}^{-}$satisfaz $B^{-}$ $(1,0) \subset B^{-}$.

Agora vamos provar um resultado acerca de $p(D) \subset A$

Lema 4.2.2: $\overline{p(D)} \supset \mathbf{S}^{1} \times\{0\}$ ou $\overline{p(D)} \supset \mathbf{S}^{1} \times\{1\}$

Demonstração: Suponha que o lema é falso. Então existem pontos $p_{0} \in \mathbf{S}^{1} \times\{0\}$ e $p_{1} \in \mathbf{S}^{1} \times\{1\}$ tais que $\left\{p_{0}, p_{1}\right\} \cap \overline{p(D)}=\emptyset$. Como $\overline{p(D)}^{c}$ é aberto, existe $\epsilon>0$ tal que $B_{\epsilon}\left(p_{i}\right) \cap \overline{p(D)}=\emptyset, i=0,1$. Como

$$
\widetilde{f}(D) \subset D \quad e \quad p \circ \widetilde{f}(\widetilde{x}, \widetilde{y})=f \circ p(\widetilde{x}, \widetilde{y})
$$

temos que, por continuidade de $f$ que

$$
f(p(D)) \subset p(D) \Rightarrow f(\overline{p(D)}) \subset \overline{p(D)} .
$$

Como $\tilde{f}$ é transitiva, $f$ também o é, logo, pelo mesmo raciocínio feito depois do Lema 4.1.1, existe $N>0$ tal que

$$
f^{-N}\left(B_{\epsilon}\left(p_{0}\right)\right) \cap B_{\epsilon}\left(p_{1}\right) \neq \emptyset
$$

e afirmamos que

$$
f^{-N}\left(B_{\epsilon}\left(p_{0}\right)\right) \cap \overline{p(D)}=\emptyset
$$

pois se isso não acontecesse, pelo falado acima, temos que $f^{N}(\overline{p(D)}) \subset \overline{p(D)}$, com o qual obtemos que $B_{\epsilon}\left(p_{0}\right) \cap \overline{p(D)} \neq \emptyset$, e isso contradiz a escolha do $\epsilon$.

Então, por tudo o que foi feito até agora, obtemos que $f^{-N}\left(B_{\epsilon}\left(p_{0}\right)\right) \cup B_{\epsilon}\left(p_{1}\right)$ é conexa e disjunta de $\overline{p(D)}$. Logo podemos assegurar que existe um arco simples e continuo $\gamma$, disjunto de $p(D)$, cujos pontos finais são $f^{-N}\left(p_{0}\right)$ e $p_{1}$.

Logo, tomando $\widetilde{\gamma}$ uma componente conexa de $p^{-1}(\gamma)$ em $\widetilde{A}$, temos que ela conecta $\mathbf{R} \times\{0\}$ com $\mathbf{R} \times\{1\}$, sendo $\widetilde{\gamma}$ um arco simples, contínuo e disjunto de $D$. Como resultado de isso, temos que $\widetilde{\gamma}-(i, 0) \cap D=\emptyset$ para todo $i$ inteiro positivo, e isto contradiz o fato de as componentes conexas de $D$ serem ilimitadas a esquerda, e obtemos assim o lema.

Para finalizar esta seção, vamos provar um resultado que será util na demonstração do teorema 2.

Lema 4.2.3 : Seja $\widetilde{f}: \widetilde{A} \rightarrow \widetilde{A}$ um homeomorfismo transitivo, $\Omega$ um aberto não vazio em $\widetilde{A}$, e suponha que $\Omega \subset \widetilde{f}(\Omega)$, então $\Omega$ é denso em $\widetilde{A}$. 
Demonstração: Suponha que $\Omega$ não é denso, então o conjunto $G=\widetilde{f}(\Omega) \backslash \Omega$ tem interior não vazio. Por construção, temos que $\widetilde{f}^{-1}(G) \subset \Omega$ e como $\widetilde{f}^{-n}(\Omega) \subset \Omega$ para todo $n$ inteiro positivo, obtemos que $\widetilde{f}^{-n}(G) \subset \Omega$ para todo $n$ inteiro positivo, por tanto $\widetilde{f}^{-n}(G) \cap G=\emptyset$. O anterior implica que $\tilde{f}^{n}(G) \cap G=\emptyset$ para todo inteiro positivo $n$. Mas como $G$ tem interior não vazio, isso contradiz, pela Afirmacão 3.1.1, o fato de $\widetilde{f}$ ser transitiva.

Note que o mesmo resultado é obtido se na hipóteses colocamos $\widetilde{f}(\Omega) \subset \Omega$.

\subsection{Demonstração do Teorema 2}

A idéia da demonstração é a seguinte: vamos supor que $\omega\left(B^{-}\right) \neq \emptyset$, fazendo uso do Lema 4.2 .2 vamos contruir um conjunto $\Omega$ aberto, satisfazendo as hipóteses do Lema 4.2.3, e daí, vamos obter uma contradição.

Suponha $\omega\left(B^{-}\right)$diferente de vazio. Pelo Lema 4.2.2, vamos ter que $\overline{p\left(\omega\left(B^{-}\right)\right)} \supset \mathbf{S}^{1} \times\{0\}$ ou $\overline{p\left(\omega\left(B^{-}\right)\right)} \supset \mathbf{S}^{1} \times\{1\}$. Vamos assumir, sem perda de generalidade, que $\overline{p\left(\omega\left(B^{-}\right)\right)} \supset \mathbf{S}^{1} \times\{0\}$.

Agora, como o número de rotação de $\widetilde{f}$ restrito a $\mathbf{S}^{1} \times\{0\}$ é estritamente positivo, temos que existe $\sigma>0$ tal que $p_{1}(\widetilde{f}(\widetilde{x}, 0))>\widetilde{x}+2 \sigma$, para todo $\widetilde{x} \in \mathbf{R}$. Pela continuidade de $\widetilde{f}$, podemos escolher $\epsilon>0$ suficientemente pequeno tal que

$$
p_{1} \circ \widetilde{f}(\widetilde{x}, \widetilde{y})>\widetilde{x}+\sigma \quad \forall(\widetilde{x}, \widetilde{y}) \in \mathbf{R} \times[0, \epsilon]
$$

Como $\mathbf{S}^{1} \times\{0\} \subset \overline{p\left(\omega\left(B^{-}\right)\right)}$, existe um numero real $a<0$ (pois $\omega\left(B^{-}\right) \subset V^{-}$) satisfazendo

$$
\omega\left(B^{-}\right) \cap\{a\} \times[0, \epsilon] \neq \emptyset
$$

Do fato de $\omega\left(B^{-}\right)$ser fechado, existe $0<\delta \leq \epsilon$ tal que o ponto $(a, \delta) \in \omega\left(B^{-}\right)$e, para todo $\widetilde{y}$, $0 \leq \widetilde{y}<\delta,(a, \widetilde{y}) \notin \omega\left(B^{-}\right)$, ou seja, $(a, \delta)$ é o ponto de $\omega\left(B^{-}\right)$mais baixo no segmento $\{a\} \times[0, \epsilon]$. Vamos denotar por $v$ ao segmento $\{a\} \times[0, \delta)$ (note que $\omega\left(B^{-}\right) \cap v=\emptyset$ ).

Tomemos $\Theta_{1}$ a componente conexa não limitada de $\omega\left(B^{-}\right)$que contém $(a, \delta)$, e seja $\Omega$ a componente conexa de $\left(\Theta_{1} \cup v\right)^{c}$ que contém $(-\infty, a) \times\{0\}$.

Pela construção, $\partial \Omega \subset \Theta_{1} \cup v$, e pelo fato de $\widetilde{f}$ ser homeomorfismo e $\left(\Theta_{1} \cup v\right)$ fechado, temos que $\partial \widetilde{f}(\Omega) \subset \widetilde{f}\left(\Theta_{1}\right) \cup \widetilde{f}(v)$.

Além disso, dado que $\omega\left(B^{-}\right) \cap v=\emptyset$ e $\omega\left(B^{-}\right)$é $\tilde{f}$ invariante temos que:

- $\widetilde{f}\left(\Theta_{1}\right) \cap v=\emptyset$ pois $\widetilde{f}\left(\Theta_{1}\right) \subset \omega\left(B^{-}\right)$(e, em geral, pelo mesmo motivo $\left.\widetilde{f}\left(\omega\left(B^{-}\right)\right) \cap v=\emptyset\right)$.

- $\Theta_{1} \cap \tilde{f}(v)=\emptyset$ pois, caso contrário, se $z \in \Theta_{1} \cap \widetilde{f}(v)$ pela invariância de $\omega\left(B^{-}\right)$teríamos uma contradição.

- $\widetilde{f}(v) \cap v=\emptyset$ pela escolha do $\epsilon$.

Então, dado que $\Omega$ é uma componente conexa de $\left(\Theta_{1} \cup v\right)^{c}$ e $\Theta_{1} \cup v$ é fechado temos que $\Omega$ é aberto, o que vamos provar agora é que $\Omega \subset \widetilde{f}(\Omega)$.

Observemos que, pelo feito acima, temos $\partial \widetilde{f}(\Omega) \subset \omega\left(B^{-}\right) \cup \widetilde{f}(v)$, e pela hipótese acerca do número de rotação de $\widetilde{f}$ na fronteira $(-\infty, a) \times\{0\} \subset \widetilde{f}((-\infty, a) \times\{0\}) \subset \widetilde{f}(\Omega), \operatorname{logo} \Omega \cap \widetilde{f}(\Omega) \neq \emptyset$. Agora, pelos items de acima, e o fato de $\widetilde{f}$ ser um homeomorfismo que preserva orientação com número de rotação positivo na fronteira, podemos assegurar que $\widetilde{f}(v) \cap \Omega=\emptyset$ e $\widetilde{f}(v) \cap \bar{\Omega}^{c} \neq \emptyset$.

Logo temos duas possibilidades (por ser $\omega\left(B^{-}\right)$união de componentes conexas e $\tilde{f}$ invariante):

- $\tilde{f}\left(\Theta_{1}\right) \cap \Theta_{1}=\emptyset$

Então temos que, $\partial \widetilde{f}(\Omega) \cap \partial \Omega=\emptyset$. Como $\left(\Theta_{1} \cup v\right) \cap \widetilde{f}(v)=\emptyset$ e $\widetilde{f}(v) \cap \bar{\Omega}^{c} \neq \emptyset$, pela continuidade de $\widetilde{f}$, obtemos que $\widetilde{f}(v) \cap \bar{\Omega}=\emptyset$. Além disso, como $\widetilde{f}\left(\Theta_{1}\right) \cap \Theta_{1}=\emptyset$, asseguramos também que $\widetilde{f}\left(\Theta_{1}\right) \cap \bar{\Omega}=\emptyset$, pois caso contrário, teríamos que $\widetilde{f}\left(\omega\left(B^{-}\right)\right) \cap v \neq \emptyset$ o qual sabemos que não 
acontece (pois $\widetilde{f}((a, \delta)) \in \widetilde{f}\left(\Theta_{1}\right)$, e qualquer entorno desse ponto tem interseção não vazia com $\widetilde{f}(v)$ e isso implicaria o de acima), portanto provamos que $\left(\widetilde{f}\left(\Theta_{1}\right) \cup \widetilde{f}(v)\right) \cap \bar{\Omega}=\emptyset$.

Agora, dado $z \in \Omega$, existe (pois as componentes conexas e as componentes conexas por curvas coinciden em $\widetilde{A}$ ) um arco continuo simples $\gamma$ com ponto inicial em $z$ e ponto final em $z_{0} \in(-\infty, a) \times$ $\{0\}$. Note que, de $(-\infty, a) \times\{0\} \subset \widetilde{f}((-\infty, a) \times\{0\}) \subset \widetilde{f}(\Omega)$ é claro que $z_{0} \in \widetilde{f}(\Omega)$, e já que $\gamma \cap\left(\Theta_{1} \cup v\right)=\emptyset$. Pelo que acabamos de provar $\gamma \cap\left(\widetilde{f}\left(\Theta_{1}\right) \cup \widetilde{f}(v)\right)=\emptyset$, logo $\gamma \subset \widetilde{f}(\Omega)$ (pois caso contrário $\gamma$ tem que interceptar a fronteira de $\widetilde{f}(\Omega))$. Como $z \in \Omega$ é arbitrário, conluimos que $\Omega \subset \widetilde{f}(\Omega)$.

- $\tilde{f}\left(\Theta_{1}\right)=\Theta_{1}$

Notemos outra vez, que, como $\left(\Theta_{1} \cup v\right) \cap \widetilde{f}(v)=\emptyset$, temos $\widetilde{f}(v) \cap \partial \Omega=\emptyset$, como $\widetilde{f}(a, 0) \in \bar{\Omega}^{c}$ então $\widetilde{f}(v) \cap \bar{\Omega}=\emptyset$.

Tomemos um arco contínuo simples $\gamma$ como no caso anterior, dado que $\gamma \cap \Theta_{1}=\gamma \cap \widetilde{f}(v)=\emptyset$, então $\gamma$ pertece a uma componente conexa de $\left(\Theta_{1} \cup \widetilde{f}(v)\right)^{c}$ como $z_{0} \in \gamma \cap \widetilde{f}(\Omega),\left(\Theta_{1} \cup \widetilde{f}(v)\right)^{c}=$ $\left(\widetilde{f}\left(\Theta_{1}\right) \cup \widetilde{f}(v)\right)^{c}, \widetilde{f}(\Omega)$ e $\gamma$ são conexos, temos que $\widetilde{f}(\Omega)$ e $\gamma$ pertecem à mesma componente conexa, mas isso diz que todo ponto $z \in \Omega$ é ponto de $\widetilde{f}(\Omega)$, portanto $\Omega \subset \widetilde{f}(\Omega)$.

Então, por todo o provado acima, temos que $\Omega$ é aberto, $\Omega \subset \widetilde{f}(\Omega)$ sendo que $\widetilde{f}$ é transitiva, concluimos, pelo Lema 4.2.3, que $\Omega$ é denso em $\widetilde{A}$, mas isso é uma contradição, pois $\Omega \subset V^{-}$. A contradição seguiu da suposição de que $\omega\left(B^{-}\right)$é não vazio, fica provado o Teorema 2.

\subsection{Demonstração do Teorema 3}

Para provar o Teorema 3, vamos provar antes um lema, e o Teorema sairá como corolário desse lema

Lema 4.4.1 Existe um inteiro positivo $N_{1}$ tal que $\widetilde{f}^{N_{1}}\left(B^{-}\right) \subset B^{-}-(1,0)$

Demonstração: Já sabemos do Teorema 2, que $\omega\left(B^{-}\right)=\emptyset$, como $\omega\left(B^{-}\right)=\bigcap_{n=0}^{\infty} \tilde{f}^{n}\left(B^{-}\right)$, e $B^{-} \subset V^{-}$, afirmamos que existe $N_{1}>0$ tal que para todo $n>N_{1}$ se satisfaz $\tilde{f}^{n}\left(B^{-}\right) \subset V_{-2}^{-}$. Pois caso contrário, temos que para todo inteiro positivo $n$ se cumpre que $\widetilde{f}^{n}\left(B^{-}\right) \cap\left(V_{-2}^{+-}\right) \neq \emptyset$ e isso contradiz o fato de ser $\omega\left(B^{-}\right)=\emptyset$. Então dado que existe $N_{1}$, temos que $\widetilde{f}^{N_{1}}\left(B^{-}\right)+(1,0) \subset V^{-}$, logo, das propriedades de $B^{-}$e o fato de $\widetilde{f}$ preservar orientação, podemos escrever

$$
\widetilde{f}\left(\widetilde{f}^{N_{1}}\left(B^{-}\right)+(1,0)\right)=\widetilde{f}^{N_{1}+1}\left(B^{-}\right)+(1,0) \subset \widetilde{f}^{N_{1}}\left(B^{-}\right)+(1,0)
$$

Isso diz que o conjunto $\widetilde{f}^{N_{1}}\left(B^{-}\right)+(1,0)$ é positivamente invariante. Além disso, como $\widetilde{f}^{N_{1}}\left(B^{-}\right) \subset$ $B^{-}$, suas componentes conexas são ilimitadas, logo as de $\widetilde{f}^{N_{1}}\left(B^{-}\right)+(1,0)$ o são, mas isso só acontece se $\widetilde{f}^{N_{1}}\left(B^{-}\right)+(1,0) \subset B^{-}$provando assim o lema.

Agora notemos que, como $\widetilde{f}^{N_{1}}\left(B^{-}\right) \subset B^{-}-(1,0)$, considerando $k$ inteiro positivo temos, por un argumento inductivo que

$$
\widetilde{f}^{k N_{1}}\left(B^{-}\right) \subset B^{-}-(k, 0)
$$

É claro que eu não posso assegurar a existência do número de rotação para qualquer $\widetilde{z} \in B^{-}$, mas eu posso limitar superiormente, para qualquer ponto nesse conjunto, seu posível valor. Lembremos que a expressão usada para calcular o número de rotação é a seguinte

$$
\frac{p_{1}\left(\widetilde{f}^{n}(\widetilde{z})\right)-p_{1}(\widetilde{\widetilde{z}})}{n}
$$

e o valor é obtido tomando o limite de $n$ tendendo a infinito. Para limitar superiormente este valor, vamos calcular o limite superior da seqüência $a_{n}$, lembrando que, se eu tenho uma seqüência $b_{n}$ qualquer, defino $c_{k}=\sup _{m \geq k}\left(b_{m}\right)$ e $\lim \sup _{n \rightarrow \infty} b_{n}=\inf _{n}\left(\sup _{k \geq n}\left(a_{k}\right)\right)=\inf _{n} c_{k}$ e que, pelas 
propridades do supremo $c_{k+1} \leq c_{k}$.

Por o feito acima, só precisamos considerar que acontece a partir de certo $n$ inteiro positivo. Seja então $n=N_{1}$.

Primeiro, notemos que para $r=0,1, \ldots, N_{1}-1$, a função $h(\widetilde{x})=\left|\widetilde{f}^{r}(\widetilde{x})-\widetilde{x}\right|$ e continua, e para qualquer $\widetilde{x} \in \widetilde{A}$ satisfaz que $h(\widetilde{x})=\left|p_{1}\left(\widetilde{f}^{r}(\widetilde{x})\right)-p_{1}(\widetilde{x})\right|<L$ para algum $L>0$ fixo e para todo $r$ em $\left\{0,1, \ldots, N_{1}-1\right\}$ (o anterior sai de considerar $h$ definida em $[0,1] \times[0,1]$ para cada $r$, logo pela continuidade num compacto obtemos que, para cada $r, h$ é limitada, e só tomamos $L$ o maior desses valores).

Agora, podemos escrever (pois estamos considerando $n>N_{1}$ ), $n=k N_{1}+r$ com $0 \leq r<N_{1}$ $k>0, \operatorname{logo}$ para qualquer $\widetilde{x} \in B^{-}$

$$
\frac{p_{1}\left(\widetilde{f}^{n}(\widetilde{x})\right)-p_{1}(\widetilde{x})}{n}=\frac{p_{1}\left(\widetilde{f}^{n}(\widetilde{x})\right)-p_{1}\left(\widetilde{f}^{k N_{1}}(\widetilde{x})\right)+p_{1}\left(\widetilde{f}^{k N_{1}}(\widetilde{x})\right)-p_{1}(\widetilde{x})}{n}
$$

e como $n=k N_{1}+r$ temos que o anterior é igual a

$$
\frac{p_{1}\left(\widetilde{f}^{r}\left(\widetilde{f}^{k N_{1}}(\widetilde{x})\right)-p_{1}\left(\widetilde{f}^{k N_{1}}(\widetilde{x})\right)+p_{1}\left(\widetilde{f}^{k N_{1}}(\widetilde{x})\right)-p_{1}(\widetilde{x})\right.}{n}
$$

e pelo feita anteriormente temos que

$$
\frac{p_{1}\left(\widetilde{f}^{r}\left(\widetilde{f}^{k N_{1}}(\widetilde{x})\right)-p_{1}\left(\widetilde{f}^{k N_{1}}(\widetilde{x})\right)+p_{1}\left(\widetilde{f}^{k N_{1}}(\widetilde{x})\right)-p_{1}(\widetilde{x})\right.}{n} \leq \frac{L}{k N_{1}+r}+\frac{-k}{k N_{1}+r}<-\frac{1}{N_{1}}
$$

Daí, pelas propriedades do limite superior concluímos a demonstração de Teorema 3.

\subsection{Demonstração do Corolário 1}

Por ser $f$ transitiva, como já sabemos, todo ponto de $A$ é não errante, logo pelo Teorema 3 e os resultados em [4], se $\widetilde{f}$ não tem pontos fixos na fronteira, o 0 esta no interior de $\rho(\widetilde{f})$.

\subsection{Propriedades de $p(D) \subset A$}

Para a definição do conjunto $\underset{\widetilde{A}}{D}$, olhar a seção 4.2. Consideramos aqui $\widetilde{f}$ transitiva, e movimentando os pontos da fronteira de $\widetilde{A}$ estritamente para direita.

Nesta seção, apresentaremos dois resultados acerca do conjunto $p(D)$ no anel, que serão de muita importância na demonstração do Teorema 4.

O primeiro dos lemas fala acerca do que acontece se $p(D)$ não é denso no anel, ou, o que é o mesmo, se $\overline{p(D)} \neq A$.

Lema 4.6.1 : Se $\overline{p(D)} \neq A$, então $(\overline{p(D)})^{c}$ é um conjunto conexo e denso em A, mais ainda, $(\overline{p(D)})^{c}$ contém uma curva fechada simples, homotopicamente não trivial em $\mathbf{S}^{1} \times(0,1)$.

Basicamente, o que diz o lema, é que se a projeção de $D$ não é densa no anel, então seu complementar é denso, e além disso, contém uma curva que separa as componentes do bordo do anel.

Demonstração: Notemos que

$$
\widetilde{f}(D) \subset D \Rightarrow p(\widetilde{f}(D)) \subset p(D)
$$

mas como $p \circ \widetilde{f}=f \circ p$ obtemos

$$
f(p(D)) \subset p(D)
$$


Mas pela continuidade de $f$, também temos que $f(\overline{p(D)}) \subset \overline{p(D)}$ Agora, como $f$ é um homeomorfismo satisfazendo o de acima e $(\overline{p(D)})^{c}=A \backslash \overline{p(D)}$ temos que

$$
f\left((\overline{p(D)})^{c}\right)=A \backslash f(\overline{p(D)}) \supset A \backslash \overline{p(D)}=(\overline{p(D)})^{c}
$$

Logo, pelo Lema 4.2.3 temos que $(p(D))^{c}$ é denso em $A$.

Agora, seja $E$ uma componente conexa de $(\overline{p(D)})^{c}$, e suponha que não exista $\gamma \subset E$ como no enunciado do lema, então, afirmamos que $p^{-1}(E)$ não é conexo. Caso contrário, se $\widetilde{x} \in p^{-1}(E)$, como ele é conexo, $\widetilde{x}-(1,0) \in p^{-1}(E)$, logo podemos construir $\gamma$ ligando $\widetilde{x}$ e $\widetilde{x}-(1,0), \gamma \subset p^{-1}(E)$, e isso contradiz a suposição. Logo, existe um conjunto $E_{\text {lift }} \subset \widetilde{A}$, tal que $p\left(E_{\text {lift }}\right)=E$, sendo $E_{\text {lift }}$ conexo e aberto (isso como consequência de $p$ ser uma aplicação contínua). Então, pelo falado acima, podemos escrever

$$
p^{-1}(E)=\bigcup_{i=-\infty}^{+\infty}\left(E_{l i f t}+(i, 0)\right)
$$

Agora, como $f$ é transitiva e $\left.f^{-1}\left((p(D))^{c}\right) \subset(p(D))^{c}\right)$, existe um primeiro inteiro $N>0$, tal que $f^{-N}(E) \subseteq E$, isso é claro, pois $f^{-m}(E) \subset(p(D))^{c}$ logo, ou a interseção com $E$ é disjunta, ou $f^{-m}(E)$ está contido no próprio $E$, pois estamos trabalhando com componentes conexas, também é satisfeito que $f^{-i}(E) \cap E=\emptyset$ para $i \in\{1,2, \ldots, N-1\}$, então, resumimos o anterior em

$$
\begin{gathered}
\widetilde{f}^{-} i\left(E_{\text {lift }}\right) \cap p^{-1}\left(\underset{\widetilde{f}^{-N}}{E}\left(E_{\text {lift }}\right) \subset E_{\text {lift }}+\left(i_{0}, 0\right),\right. \\
\text { para algum inteiro fixo } i_{0}, \text { portanto } \\
\widetilde{f}^{k . N}\left(E_{\text {lift }}\right) \subset E_{\text {lift }}+\left(k \cdot i_{0}, 0\right), \text { para todo inteiro } k<0 .
\end{gathered}
$$

Agora vamos provar que o anterior não pode acontecer. Suponha $i_{0}>0$. Como $\tilde{f}$ tem uma órbita densa, existe $\widetilde{z} \in E_{\text {lift }}-(1,0)$ tal que $\widetilde{f}^{l}(\widetilde{z}) \in E_{\text {lift }}$, onde, pelo já feito antes, posso supor $l>0$. Então temos que $\widetilde{f}^{l}\left(E_{\text {lift }}-(1,0)\right) \cap E_{\text {lift }} \neq \emptyset \operatorname{logo}$

$$
\tilde{f}^{-l}\left(E_{l i f t}\right) \cap\left(E_{l i f t}-(1,0)\right) \neq \emptyset
$$

O que contradiz nossa afirmação anterior, pois $i_{0}$ é maior que 0 . Como o mesmo é válido se escolhemos $i_{0}$ menor que 0 , obtemos uma contradição, que provem de supor a não existencia de $\gamma$ nas condições do lema. Logo toda componente conexa $E \subset(p(D))^{c}$ contém uma curva fechada simples, homotopicamente não trivial $\gamma_{E}$.

O único que resta a provar é que $(p(D))^{c}$ possui uma única componente conexa, mas isso sai do fato de que, pela transitividade de $f, f^{-1}\left(\gamma_{E}\right) \cap \gamma_{E} \neq \emptyset$. Caso contrário, sejam $\gamma_{E}^{+}$e $\gamma_{E}^{-}$as duas componentes conexas de $\left(\gamma_{E}\right)^{c}$, se $f^{-1}\left(\gamma_{E}\right) \cap \gamma_{E}=\emptyset$, então a imagem de uma das componentes conexas, suponha $\gamma_{E}^{+}$, satisfaz que

$$
f^{-1}\left(\gamma_{E}^{+}\right) \subset \gamma_{E}^{+}
$$

O qual contradiz a transitividade de $f$, pois os pontos em $\gamma_{E}^{+}$nunca ficam fora desse conjunto (Lema 4.2.3).

Agora seja

$$
\gamma_{E} \subset(p(D))^{c}-\left\{\mathbf{S}^{1} \times\{0\} ; \mathbf{S}^{1} \times\{1\}\right\}
$$

uma curva fechada, simples, homotopicamente não trivial. Vamos dar una definição mais formal das componentes conexas de $\left(\gamma_{E}\right)^{c}$. Como são duas componentes conexas, definimos $\gamma_{E}^{-}$como a componente conexa de $\left(\gamma_{E}\right)^{c}$ que contém $\mathbf{S}^{1} \times\{0\}$ e $\gamma_{E}^{+}$como a componente conexa de $\left(\gamma_{E}\right)^{c}$ que contém $\mathbf{S}^{1} \times\{1\}$.

Concluiremos esta seção como o seguinte lema

Lema 4.6.2 : Seja $\Gamma$ uma componente conexa de $D$, se $\overline{p(D)} \neq A$, temos que 


$$
\begin{aligned}
& \text { se } \overline{p(\Gamma)} \subset \gamma_{E}^{-}, \text {então } \overline{p(\Gamma)} \supset \mathbf{S}^{1} \times\{0\} \\
& \text { se } \overline{p(\Gamma)} \subset \gamma_{E}^{+}, \text {então } \overline{p(\Gamma)} \supset \mathbf{S}^{1} \times\{1\}
\end{aligned}
$$

Demonstração: Notemos que como $p(\Gamma) \subset p(D)$, então $\overline{p(\Gamma)} \subset \overline{p(D)}$ e $(\overline{p(\Gamma)})^{c} \supset(\overline{p(D)})^{c}$. Logo, $(\overline{p(\Gamma)})^{c}$ é aberto e contém um conjunto conexo e denso, logo, pelo mesmo raciocínio do teorema anterior, $(\overline{p(\Gamma)})^{c}$ é aberto, conexo e denso.

Agora suponha $\overline{p(\Gamma)} \subset \gamma_{E}^{-}$(a idéia para o outro caso é a mesma). Como $\gamma_{E}$ é fechada. simples e homotopicamente não trivial, contida no interior do anel, é claro que $\overline{p(\Gamma)} \cap \mathbf{S}^{1} \times\{1\}=\emptyset$. Suponha que o lema não vale. Como $(\overline{p(\Gamma)})^{c}$ é aberto e conexo, temos que, se $\overline{p(\Gamma)}$ não contém $\mathbf{S}^{1} \times\{0\}$, então existem $p_{0} \in \mathbf{S}^{1} \times\{0\}$ e $\epsilon$ positivo tal que $B_{\epsilon}\left(p_{0}\right) \cap \overline{p(\Gamma)}=\emptyset$. Portanto, pelo feito no lema 4.2.2, existe $\alpha$ um arco no anel, como pontos extremos em $p_{0} \in \mathbf{S}^{1} \times\{0\}$ e $p_{1} \in \mathbf{S}^{1} \times\{1\}$ satisfazendo que $\alpha \cap p(\Gamma)=\emptyset$. Mas todo o anterior implica que

$$
p^{-1}(\alpha) \cap \Gamma=\emptyset
$$

Agora, como cada componente conexa de $p^{-1}(\alpha)$ é fechada, e une pontos na fronteira da faixa, isso contradiz o fato de $\Gamma$ ser ilimitada a esquerda. Obtemos assim uma contradição que prova o lema 


\section{Capítulo 5}

\section{Da estrutura de $D \subset \widetilde{A}$}

Começamos lembrando as propriedades que satisfaz o conjunto fechado e não vazio $D$ :

- $\widetilde{f}(D) \subset D$.

- $D \subset V^{-}$.

- Toda componente conexa de $D$ é ilimitada a esquerda.

- $D \cap \mathbf{R} \times\{i\}=\emptyset, i \in\{0,1\}$.

- $\widetilde{z} \in D$ então $\widetilde{z}-(1,0) \in D$.

\subsection{Resultados preliminares}

A idéia desta seção, é definir uma relação de ordem nas componentes conexas de $D$, e considerar certas possibilidades que podem acontecer nestas componentes conexas, mais precisamente, consideramos o caso em que a projeção sobre o anel é ou não é injetora.

O trabalho a realizar vai ser um pouco mais geral, e vai permitir definir um ordem nos conjuntos fechados conexos ilimitados de $V^{-}$que tem complementar conexo. Vamos começar provando certos resultados que serão de utilidade em nossa definição de ordem.

Proposição 5.1.1 : Seja $\Gamma$ uma componente conexa de $D$, então $\Gamma^{c}$ possui só uma componente conexa, a qual é ilimitada.

Demonstração: Lembremos que $\Gamma$ é ilimitada a esquerda, $\Gamma \subset V^{-}$, e $\Gamma \cap \mathbf{R} \times\{i\}=\emptyset$ para $i=0,1$. Logo é claro que existe uma componente conexa ilimitada de $\Gamma^{c}$ que contém $\operatorname{int}\left(V^{-}\right), \mathbf{R} \times\{0\}$ e $\mathbf{R} \times\{1\}$.

Suponha agora que $\Gamma^{c}$ tivesse outra componente conexa, denotada por $C$, contida em $V^{-}$. Como $C \subset \Gamma^{c}$, sua fronteira tem que estar contida em $\Gamma$. Pelas propriedades de $D, \widetilde{f}^{n}(\Gamma) \subset V^{-}$para todo inteiro positivo $n$.

Como $\partial C \subset \Gamma \subset V^{-}$e $\widetilde{f}$ é um homeomorfismo, temos que $\partial \widetilde{f}(C) \subset \widetilde{f}(\partial C) \subset V^{-}$e pelo fato de $\widetilde{f}$ preservar orientação, temos que $\widetilde{f}(C) \subset V^{-}$. Mas isso, pelo mesmos argumentos trablhados antes, contradiz o fato de ter $\widetilde{f}$ uma órbita densa, pois os pontos de $C$ permanecen em $V^{-}$ao longo de iteradas positivas de $f$. Esta contradição prova a proposição.

Definição: Para uma componente conexa $\Gamma$ de $D$, definimos

$$
m_{\Gamma}=\sup \{\widetilde{x} \in \mathbf{R}:(\widetilde{x}, \widetilde{y}) \in \Gamma \text { para algum } \widetilde{y} \in[0,1]\} .
$$

Da definição anterior, consideramos o seguinte conjunto conexo e fechado

$$
\Gamma \cup\left\{m_{\Gamma}\right\} \times[0,1]
$$


Claramente, seu complementar possui, pelo menos, duas componentes conexas abertas e ilimitadas em $\left(-\infty, m_{\Gamma}\right) \times[0,1]$, uma denotada por $\Gamma_{\text {down }}$ que contém $\left(-\infty \cdot m_{\Gamma}\right) \times\{0\}$ e a outra, denotada $\Gamma_{u p}$, que contém $\left(-\infty, m_{\Gamma}\right) \times\{1\}$. É claro que existe a possibilidade de $\left(\Gamma \cup\left\{m_{\Gamma}\right\} \times[0,1]\right)^{c}$ ter outras componenetes conexas ilimitadas, mas só estas duas componentes conexas ilimitadas nos interessam, devido à seguinte afirmação:

Afirmação 5.1.1 : Dada uma componente conexa $\Gamma$ de $D$, se $\Theta$ é um conjunto fechado e conexo, ilimitado a esquerda, que satisfaz $\Theta \cap \Gamma=\emptyset e \Theta \subset\left(-\infty, m_{\Gamma}\right) \times[0,1]$, então $\Theta \subset \Gamma_{\text {up }}$ ou $\Theta \subset \Gamma_{\text {down }}$.

A prova da afirmação anterior vai ser feita imediatamente depois da prova do Lema 5.1.2, que prova um caso mais geral do anterior.

A idéia agora é definir uma relação de ordem nas componentes conexas de $D$, mas o trabalho vai ser mais geral, isto é, vamos definir uma relação de ordem sobre os conjuntos conexos, disjuntos, fechados e ilimitados a esquerda de $V^{-}$, com complementar conexo. O motivo par fazer isso é que, ainda que tivesemos $\Gamma_{1}, \Gamma_{2}$ duas compoenentes conexas de $D, \widetilde{f}\left(\Gamma_{1}\right)$ e $\widetilde{f}\left(\Gamma_{2}\right)$ podem pertencer a uma mesma componente conexa. Então é preciso definir uma relação de ordem mais geral, e vamos mostrar que $\tilde{f}$ preserva a ordem.

Começamos tomando $\Gamma \subset D$ uma componente conexa e $a \leq 0$ tal que $V_{a} \cap \Gamma \neq \emptyset$. Consideramos

$$
\Gamma^{c o m p, a}=\Gamma^{c} \cap((-\infty, a) \times[0,1])
$$

Então

Lema 5.1.1 : $\Gamma^{c o m p, a}$ tem, pelo menos duas componentes conexas abertas, uma denotada por $\Gamma_{a, d o w n}^{\prime}$ que contém $(-\infty, a) \times\{0\}$ e a outra, denotada por $\Gamma_{a, u p}^{\prime}$ que contém $(-\infty, a) \times\{1\}$.

Demonstração: Suponha que o lema é falso, Então $(-\infty, a) \times\{0\}$ e $(-\infty, a) \times\{1\}$ estão na mesma componente conexa, e existem $P \in(-\infty, a) \times\{0\}, Q \in(-\infty, a) \times\{1\}$, e um arco continuo simples $\eta \in \Gamma^{\text {comp,a }}$ com pontos finais $P$ e $Q$. Logo, pela construção $\eta \subset(-\infty, a) \times[0,1]$, e $\eta^{c}$ tem duas componenetes conexas em $(-\infty, a) \times[0,1]$. Como $\Gamma \cap \eta=\emptyset, \Gamma \cap V_{a} \neq \emptyset$ e $\Gamma$ é ilimitada a esquerda, temos que $\Gamma$ está contida nas duas componentes disjuntas de $\eta^{c}$, o que contradiz a conexidade de $\Gamma$, e prova o lema.

Vamos provar um resultado acerca das componentes conexas de $D$ e sua interseção com os segmentos verticais da forma $V_{a}$.

Proposição 5.1.2 : Seja $\Gamma \subset D$ uma componente conexa de $D$, e seja $a \leq 0$ tal que $V_{a}$ intersecta $\Gamma$. Então $\Gamma \cap V_{a}^{-}$tem, pelo menos, uma componente conexa ilimitada, que intersecta $V_{a}$.

Demonstração: Como antes, vamos considerar a $L-R$ compactificação de $\widetilde{A}$, denotada por $\widehat{A}$, e qualquer elemento em $\widehat{A}$ será denotado com o $\wedge$ acima.

Seja agora $z_{n} \in \Gamma \cap V_{a}^{-}$uma seqüência tal que $p_{1}\left(z_{n}\right) \rightarrow-\infty$ quando $n \rightarrow \infty$, ou, o que é o mesmo, $\widehat{z_{n}} \rightarrow L$ em $\widehat{A}$. Lembremos que $\widehat{\Gamma}$ é conexo, intersecta $\widehat{V}_{a}^{-}$e contém $L$. Notemos que, no caso mais geral, $\Gamma \cap V_{a}^{-}$pode ter mais de uma componente conexa, alguma das quais podem até não ser ilimitadas, agora, é claro que as componentes conexas ilimitadas de $\Gamma \cap V_{a}^{-}$intersectam $V_{a}^{-}$, pois caso contrário, se $M$ é uma componente conexa propria e ilimitada de $\Gamma \cap V_{a}^{-}$que não corta $V_{a}$ temos que $M \cap \Gamma \backslash M=\emptyset$, mas isso implica que $\Gamma$ não é conexo, o qual é uma contradição. Também, pelo mesmo motivo, se eu tivese uma componente conexa de $\Gamma \cap V_{a}^{-}, N$, limitada, $N$ intercepta $V_{a}$.

Pelo falado acima, cada $\widehat{z}_{n}$ pertence a uma componente conexa de $\Gamma \cap V_{a}^{-}$, chamada $\widehat{\Gamma}_{n}$, que intercepta $\widehat{V}_{a}$. Como já provamos no començo do trabalho, existe uma subseqüência $\left\{\widehat{\Gamma}_{n_{i}}\right\}$ convergente a um elemento $\widehat{\Gamma}^{*}$., com a propriedad de que $\widehat{\Gamma}^{*}$ contém $L$ (pela escolha da seqüência $\widehat{z}_{n_{i}}$ ), e 
intercepta $\widehat{V}_{a}$.

O que falta provar e que $\widehat{\Gamma}^{*} \subset \widehat{\Gamma}$. Notemos $\widehat{\Gamma}_{n_{i}} \rightarrow \widehat{\Gamma}^{*}$, implica que para todo $\epsilon>0$ e $n_{i}$ suficientemente grande, um $\frac{\epsilon}{2}$ entorno de $\widehat{\Gamma}_{n_{i}}$ contém $\widehat{\Gamma}^{*}$. Suponha que $\widehat{\Gamma}^{*}$ não esteja contida em $\widehat{\Gamma}$, logo por ser $\widehat{\Gamma}$ fechado, temos existe $\widehat{B}_{\epsilon_{0}}(\widehat{p})$ tal que $\widehat{B}_{\epsilon_{0}}(\widehat{p}) \cap \widehat{\Gamma}=\emptyset$, para algum $\widehat{p} \in \widehat{\Gamma}^{*}$ e algum $\epsilon_{0}>0$. Sendo que $\widehat{B}_{\epsilon_{0}}(\widehat{p})$ é a bola métrica com a distância euclídea usual. Mas tomando $n_{j}$ suficientemente grande, pelo falado acima $\widehat{\Gamma}^{*}$ e $\widehat{\Gamma}_{n_{j}}$ estão contidos num $\frac{\epsilon_{0}}{2}-$ entorno $\widehat{U}$ de $\widehat{\Gamma}_{n_{j}}$, mas isso implica que a distância de $\widehat{p}$ a $\widehat{\Gamma}$ é menor que a distância de $\widehat{p}$ a $\widehat{\Gamma}_{n_{j}}$ que é menor a $\frac{\epsilon_{0}}{2}$ o qual contradiz a escolha do $\frac{\epsilon_{0}}{2}, \operatorname{logo}$ tomando uma componente conexa de $\Gamma^{*}$ que intercepte $V_{a}$ temos a proposição provada

Vamos definir os seguintes conjuntos, $\Gamma_{a, \text { down }}$ e $\Gamma_{a, u p}$ do seguinte modo

$$
\begin{gathered}
\Gamma_{a, \text { down }}=\Gamma_{a, \text { down }}^{\prime} \cup\left\{\text { todos os pontos em } \partial \Gamma_{a, \text { down }}^{\prime} \text { da forma }(a, \widetilde{y})\right\} \\
\Gamma_{a, \text { up }}=\Gamma_{a, u p}^{\prime} \cup\left\{\text { todos os pontos em } \partial \Gamma_{a, u p}^{\prime} \text { da forma }(a, \widetilde{y})\right\}
\end{gathered}
$$

E como notamos antes, se $\Gamma$ é uma componente conexa de $D$, a qual intersecta algum $V_{a}$, é posível que $\Gamma \cap V_{a}^{-}$tenha mais de uma componente conexa ilimitada, então denotamos por

$\left[\Gamma \cap V_{a}^{-}\right]=$União de todas as componentes conexas ilimitadas de $\Gamma \cap V_{a}^{-}$.

Os seguintes resultados mostram o caminho para definir a relação de ordem procurada

Proposição 5.1.3 : Sejam $a, b \leq 0$, tais que $b<a$, e seja $\Gamma$ uma componente conexa de $D$ que intersecta $V_{a}$. Então $\Gamma_{b, \text { down }} \subset \Gamma_{a, \text { down }} e \Gamma_{b, u p} \subset \Gamma_{a, \text { up }}$.

Demonstração: Vamos trabalhar com $\Gamma_{b, d o w n}$, a outra inclusão é provada da mesma forma. Seja $\widetilde{z} \in \Gamma_{b, \text { down }}$. Por definição de $\Gamma_{b, \text { down }}$, existe um arco continuo simples $\theta$ que conecta $\widetilde{z}$ a $p_{0} \in$ $(-\infty, b) \times\{0\}$. É claro que podemos escolher $\theta$ de forma que $\theta \cap \Gamma=\emptyset$ e $\theta \subset \Gamma_{b, \text { down }} \subset(-\infty, b) \times[0,1]$. Como $a>b$, temos que o único que fica de $\theta \cap \partial \Gamma_{a, \text { down }}$ é $\theta \cap \Gamma$, mas $\theta \cap \Gamma=\emptyset$. Como $p_{0} \in \Gamma_{a, d o w n}$, obtemos que $\theta \subset \Gamma_{a, \text { down }}$, dado que $\widetilde{z}$ é arbitrário, e as componentes conexas por curvas são as componentes conexas em $D$, provamos que $\Gamma_{b \text {,down }} \subset \Gamma_{a, \text { down }}$, que era o resultado procurado.

Sejam agora $\Gamma_{1}$ e $\Gamma_{2}$ duas componentes conexas distintas de $D$, e $V_{a}$ tal que $V_{a} \cap \Gamma_{1} \neq \emptyset$ então temos que:

Lema 5.1.2 : Uma e só uma das seguintes possibilidades é satisfeita:

$$
\left[\Gamma_{2} \cap V_{a}^{-}\right] \subset \Gamma_{1 a, \text { down }} \text { ou }\left[\Gamma_{2} \cap V_{a}^{-}\right] \subset \Gamma_{1 a, u p} .
$$

Demonstração: A idéia geométrica do lema é simples, dadas duas componentes conexas de $D$, se algum segmento da forma $V_{a}$ intercepta $\Gamma_{1}$, então todas as componentes conexas ilimitadas da interseção de $\Gamma_{2}$ com o segmento $V_{a}$, estão contidas na componente conexa de $\Gamma_{1}^{c o m p}, a$ que contém $(-\infty, a) \times\{0\}$ ou estão contidas na componente conexa que contém $(-\infty, a) \times\{1\}$.

A idéia da demonstração, é provar, primeiro que $\left[\Gamma_{2} \cap V_{a}^{-}\right] \subset \Gamma_{1 a, \text { down }} \cup \Gamma_{1 a \text {,up }}$, e logo, provado isso, supondo que existem componentes conexas em $\Gamma_{1 a \text {,down }}$ e $\Gamma_{1 a, u p}$ achar uma contradição que prova o buscado.

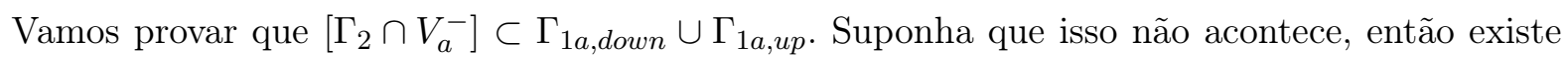
uma componente conexa ilimitada de $\Gamma_{2} \cap V_{a}^{-}$, denotada por $\Gamma_{2}^{*}$, contida numa componente conexa de $\Gamma_{1}^{c o m p, a}$, diferente de $\Gamma_{1 a, \text { down }}$ e $\Gamma_{1 a, u p}$. Denotemos essa componente conexa por $\Gamma_{1 a, m i d}$.

Fixemos $p \in \Gamma_{2}^{*}$. Como $p \notin \Gamma_{1}$, existe $\epsilon>0$ tal que $B_{\epsilon}(p) \cap \Gamma_{1}=\emptyset$. Agora, tomemos $\alpha^{\prime} \subset \mathbf{R} \times(0,1)$ uma curva simples e continua que conecta $p$ como o ponto $(1,0.5)$ (note que $(1,0.5) \notin V^{-}$), e vamos tomar $\alpha^{\prime}$ tal que ela esteja contida em $\Gamma_{1}^{c}$. Lembremos que $\Gamma_{1}^{c}$ é aberto, conexo e contém $p$ e $(1,0.5)$. Para favorecer nossa construção, dado que $\Gamma_{1}^{c} \supset(0,+\infty) \times[0,1]$, vamos também a tomar $\alpha^{\prime}$ satisfazendo que $\alpha^{\prime} \cap(1,+\infty) \times\{0.5\}=\emptyset$.

Feito o anterior, vamos definir $\alpha$ como o arco continuo simples dado pela semirecta $[1,+\infty) \times\{0.5\}$, mais uma parte continua de $\alpha^{\prime}$, com pontos finais $(1,0.5)$ e algum ponto em $\Gamma_{2}^{*}$ de modo que $\alpha \cap \Gamma_{2}^{*}$ consista só do ponto final da parte continua de $\alpha^{\prime}$ que não é $(1,0.5)$ (obviamente, por nossa escolha e todo o falado acima, esse ponto não pode ser $p$ ).

Por construção, o conjunto $\alpha \cup \Gamma_{2}^{*}$ tem as seguintes propriedades: 
- $\alpha \cup \Gamma_{2}^{*}$ é conexo, fechado e disjunto de $\mathbf{R} \times\{0\}$ e $\mathbf{R} \times\{1\}$.

- $\mathbf{R} \times\{0\}$ e $\mathbf{R} \times\{1\}$ estão em diferentes componentes conexas de $\left(\alpha \cup \Gamma_{2}^{*}\right)^{c}$.

- $\alpha$ é limitada a esquerda, ou seja, existe $M>0$ tal que para todo $\widetilde{z} \in \alpha, p_{1}(\widetilde{z})>-M$.

- $\left(\alpha \cup \Gamma_{2}^{*}\right) \cap \Gamma_{1}=\emptyset$.

A menos obvia das propriedades é a terceira, mas sai do fato de $\Gamma_{2}$ ser limitado a direita.

Agora, escolha $b<a$ tal que $\alpha \subset V_{b+\frac{1}{2}}^{+}$. Pela proposição 5.1.3, temos que $\Gamma_{1 b \text {,down }} \subset \Gamma_{1 a, \text { down }}$ e $\Gamma_{1 b, u p} \subset \Gamma_{1 a, u p}$. Por a suposição feita ao començo temos que

$$
\Gamma_{2}^{*} \cap\left(\Gamma_{1 b, \text { down }} \cup \Gamma_{1 b, \text { up }}\right)=\emptyset
$$

Pelas propriedades dos conjuntos, podemos escolher $\beta_{0} \subset \Gamma_{1 b \text {,down }}$ e $\beta_{1} \subset \Gamma_{1 b \text {,up }}$ arcos continuos simples satisfazendo o seguinte:

- $\beta_{0}$ conecta um ponto de $(-\infty, b) \times\{0\}$ a um ponto de $\Gamma_{1}$.

- $\beta_{1}$ conecta um ponto de $(-\infty, b) \times\{1\}$ a um ponto de $\Gamma_{1}$.

É claro que podemos tomar $\beta_{i} \subset V_{b}^{-}, i=0,1$. Então, por construção de $\alpha$ e a escolha de $b$, a seguinte implicação

$$
\left(\beta_{0} \cup \beta_{1}\right) \cap \Gamma_{2}^{*}=\emptyset \text { e }\left(\beta_{0} \cup \beta_{1}\right) \subset V_{b}^{-} \Rightarrow\left(\beta_{0} \cup \beta_{1}\right) \cap \alpha=\emptyset .
$$

Logo, como $\Gamma_{2}^{*} \cap \Gamma_{1}=\emptyset$ e $\alpha \cap \Gamma_{1}=\emptyset$, temos que o conjunto $\beta_{0} \cup \Gamma_{1} \cup \beta_{1}$ é um conjunto conexo, fechado e

$$
\left(\beta_{0} \cup \Gamma_{1} \cup \beta_{1}\right) \cap\left(\alpha \cup \Gamma_{2}^{*}\right)=\emptyset
$$

Mas $\beta_{0} \cup \Gamma_{1} \cup \beta_{1}$ contém pontos de $\mathbf{R} \times\{0\}$ e $\mathbf{R} \times\{1\}$, e isso contradiz o fato de $\beta_{0} \cup \Gamma_{1} \cup \beta_{1}$ ser um conjunto fechado, conexo, e $\mathbf{R} \times\{0\}$ e $\mathbf{R} \times\{1\}$ estar em diferentes componentes conexas de $\left(\alpha \cup \Gamma_{2}^{*}\right)^{c}$. Esta contradição mostra que

$$
\left[\Gamma_{2} \cap V_{a}^{-}\right] \subset \Gamma_{1 a, \text { down }} \cup \Gamma_{1 a, \text { up }}
$$

Provado o anterior, vamos supor que para $\Gamma_{2}^{*}, \Gamma_{2}^{* *} \subset\left[\Gamma_{2} \cap V_{2}^{-}\right]$, temos que $\Gamma_{2}^{*} \subset \Gamma_{1 a, \text { down }}$ e $\Gamma_{2}^{* *} \subset$ $\Gamma_{1 a, \text { up. }}$

Fazendo a mesma construção que antes, existe uma curva continua simples $\alpha \subset \mathbf{R} \times(0,1)$, que contém $[0,+\infty) \times\{0.5\}$ e conecta algum ponto de $\Gamma_{1}$ ao ponto $(1,0.5)$, de modo que $\alpha \subset \Gamma_{2}^{c}$ e intersecta $\Gamma_{1}$ so no seu ponto final diferente de $(1,0.5)$. Outra vez, por construção, temos que $\mathbf{R} \times\{0\}$ e $\mathbf{R} \times\{1\}$ estão em diferentes componentes conexas de $\left(\alpha \cup \Gamma_{1}\right)^{c}$.

Agora, novamente, escolha $b<a$ tal que $\alpha \subset V_{b+1}^{+}$. O fato de ter $\Gamma_{2}^{*}, \Gamma_{2}^{* *} \subset\left[\Gamma_{2} \cap V_{2}^{-}\right]$, e a demonstração da Proposição 5.1.2, implica, já que $b<a$, que $\Gamma_{2}^{*}$ e $\Gamma_{2}^{* *}$ interceptam $V_{b}$, e portanto $\left[\Gamma_{2}^{*} \cap V_{b}^{-}\right]$e $\left[\Gamma_{2}^{* *} \cap V_{b}^{-}\right]$são não vazios, e pelo já feito, temos que

$$
\left[\Gamma_{2}^{*} \cap V_{b}^{-}\right] \cup\left[\Gamma_{2}^{* *} \cap V_{b}^{-}\right] \subset \Gamma_{1 b, \text { down }} \cup \Gamma_{1 b, u p}
$$

Suponha que $\left[\Gamma_{2}^{*} \cap V_{b}^{-}\right] \cap \Gamma_{1 b, u p} \neq \emptyset$, logo temos que $\Gamma_{2}^{*} \cap \Gamma_{1 b, u p} \neq \emptyset$, o que implica, pela Proposição 5.1.2, que $\Gamma_{2}^{*} \cap \Gamma_{1 a, u p} \neq \emptyset$, o que, pela escolha de $\Gamma_{2}^{*}$, é uma contradição. Logo $\left[\Gamma_{2}^{*} \cap V_{b}^{-}\right] \cap \Gamma_{1 b, u p}=\emptyset$, logo também temos que $\left[\Gamma_{2}^{* *} \cap V_{b}^{-}\right] \cap \Gamma_{1 b \text {,down }}=\emptyset$.

Então, podemos tomar $\beta_{0}$ um arco continuo simples, com pontos finais em $\Gamma_{2}^{*}$ e $(-\infty, b) \times\{0\}$, totalmente contido em $\Gamma_{1 b \text {,down. }}$ E $\beta_{1}$ continuo simples com pontos finais em $\Gamma_{2}^{* *}$ e $(-\infty, b) \times\{1\}$ contido totalmente em $\Gamma_{1 b, u p}$. Agora, $\beta_{0} \cup \Gamma_{2} \cup \beta_{1}$ é um conjunto conexo, fechado, contendo pontos de $\mathbf{R} \times\{0\}$ e $\mathbf{R} \times\{1\}$ e, por construção

$$
\left(\beta_{0} \cup \Gamma_{2} \cup \beta_{1}\right) \cap\left(\alpha \cup \Gamma_{1}\right)=\emptyset
$$


A qual da a mesma contradição de antes, provando o Lema.

Vamos provar agora, usando o lema anterior, a afirmação 5.1.1, feita ao princípio do capítulo. A prova sai de considerar $V_{a}=V_{m_{\Gamma}}$, e note que, no lema anterior, o fato de $\Gamma_{1}$ e $\Gamma_{2}$ pertencer a $D$, é para assegurar que ambos conjuntos são conexos, fechados, disjuntos e contidos em $V^{-}$, mas as hipóteses da nossa afirmação, implica que $\Theta$ e $\Gamma$ satisfazem as propriedades pedidas para $V_{a}=V_{m_{\Gamma}}$. Agora, por nossa escolha (e por construção) temos que $\left[\Theta \cap V_{m_{\Gamma}}^{-}\right] \subset \Gamma_{m_{\Gamma}, \text { down }}=\Gamma_{\text {down }}$ ou $\left[\Theta \cap V_{m_{\Gamma}}^{-}\right] \subset \Gamma_{m_{\Gamma}, \text { up }}=\Gamma_{\text {up }}$. Mas como $\Theta \cap \Gamma=\emptyset$ e $\Theta \subset\left(-\infty, m_{\Gamma}\right) \times[0,1]$, sendo ele fechado, obtemos $\left[\Theta \cap V_{a}^{-}\right]=\Theta$, provando assim a afirmação 5.1.1.

Lembrando que nosso objetivo é definir uma relação de ordem sobre as componentes conexas de $D$, temos que os resultados anteriores serviram para justificar a seguinte definição.

Definição: Sejam $\Gamma_{1}$ e $\Gamma_{2}$ diferentes componentes conexas de $D$, e $a \leq 0$ tal que $\Gamma_{1}$ e $\Gamma_{2}$ interceptam $V_{a}$. Dizemos que

$$
\Gamma_{2} \prec_{a} \Gamma_{1} \text { se }\left[\Gamma_{2} \cap V_{a}^{-}\right] \subset \Gamma_{1 a, \text { down }}
$$

O próximo passo, é mostrar que a definição anterior, é precisamente uma boa definição para a relação de ordem procurada. A primeira cosa que vamos provar é que a definição independe de $a$, e só precisamos que $V_{a}$ intercepte $\Gamma_{1}$ e $\Gamma_{2}$. Para isso vamos provar o seguinte:

Lema 5.1.3 : Dadas $\Gamma_{1}$ e $\Gamma_{2}$ componentes conexas distintas de $D$, e $a \leq 0$, tal que $V_{a} \cap \Gamma_{i} \neq \emptyset$ $(i=1,2)$, temos que se cumpre uma e só uma das seguintes possibilidades:

$$
\Gamma_{2} \prec_{a} \Gamma_{1} \text { ou } \Gamma_{1} \prec_{a} \Gamma_{2}
$$

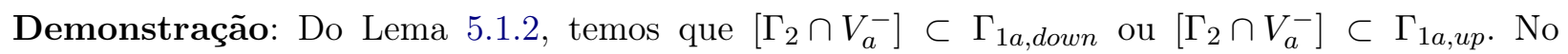
primeiro caso, por definição temos que $\Gamma_{2} \prec{ }_{a} \Gamma_{1}$. Logo, só temos que provar que, se $\left[\Gamma_{2} \cap V_{a}^{-}\right] \subset$ $\Gamma_{1 a, u p}$, então $\Gamma_{1} \prec_{a} \Gamma_{2}$.

Como, pelo Lema 5.1.2 $\left[\Gamma_{1} \cap V_{a}^{-}\right] \subset \Gamma_{2 a, \text { down }}$ ou $\left[\Gamma_{1} \cap V_{a}^{-}\right] \subset \Gamma_{2 a, \text { up }}$, vamos supor que $\left[\Gamma_{2} \cap V_{a}^{-}\right] \subset$ $\Gamma_{1 a, u p}$ e que $\left[\Gamma_{1} \cap V_{a}^{-}\right] \subset \Gamma_{2 a, u p}$, a idéia é achar uma contradição. Então, supondo o anterior, vamos trablahar na mesma forma que no Lema 5.1.2.

Começamos tomando um arco continuo simples $\alpha \subset \mathbf{R} \times(0,1)$, como na demonstração do Lema 5.1.2, de modo que $\alpha \subset \Gamma_{2}^{c}$ e intercepte $\Gamma_{1}$ só no ponto final de $\alpha$ que não é $(1,0.5)$. Outra vez, temos que $\left(\alpha \cup \Gamma_{1}\right)$ é um conjunto fechado conexo tal que $\mathbf{R} \times\{1\}$ e $\mathbf{R} \times\{0\}$ estão em diferentes componentes conexas de $\left(\alpha \cup \Gamma_{1}\right)^{c}$.

Como $\left[\Gamma_{2} \cap V_{a}^{-}\right] \subset \Gamma_{1 a, u p}$, existe um elemento $\Gamma_{2}^{*} \in\left[\Gamma_{2} \cap V_{a}^{-}\right]$, que, por definição, é fechado, conexo, ilimitado a esquerda, e $\Gamma_{2}^{*} \subset \Gamma_{1 a, u p}$. Novamente, tomemos $b<a$ tal que $\alpha \subset V_{b+1}^{+}$.

Notemos que, da demonstração do Lema 5.1.2, obtemos que $\left[\Gamma_{2}^{*} \cap V_{b}^{-}\right] \subset \Gamma_{1 b, u p}$, e podemos escolher um arco continuos simples $\beta_{1} \subset \Gamma_{1 b \text {,up }}$ que conecta um ponto de $\Gamma_{2}^{*}$ a algum ponto em $(-\infty, b) \times\{1\}$. Além disso, por construção, temos que $\beta_{1} \cap \Gamma_{1}=\emptyset$.

Agora, da nossa suposição, temos $\left[\Gamma_{1} \cap V_{a}^{-}\right] \subset \Gamma_{2 a, u p}$, então temos a seguinte:

Afirmação 5.1.2 : Existe um numero real $c \leq b$ tal que $\left(\Gamma_{1} \cap V_{c}^{-}\right) \cap \Gamma_{2 a, \text { down }}=\emptyset$.

Prova: Suponha à afirmação falsa, logo temos uma seqüência de pontos $z_{n} \in \Gamma_{1} \cap \Gamma_{2 a, \text { down }}$ tais que $p_{1}\left(z_{n}\right) \rightarrow-\infty$ se $n \rightarrow \infty$ (note que o anterior é certo pois estamos supondo a afirmação falsa), ou equivalentemente, $\widehat{z}_{n} \in \widehat{A}$ e $\widehat{z}_{n} \rightarrow L$. É claro, pelo mesmo argumento de antes (Proposição 5.1.2), que cada $\widehat{z}_{n}$ pertence a uma componente conexa de $\widehat{\Gamma}_{1} \cap \widehat{V}_{a}^{-}$, denotada $\widehat{\Gamma}_{1, n}$ que por construção satisfaz $\widehat{\Gamma}_{1, n} \subset \overline{\widehat{\Gamma}_{2 a, \text { down }}}$.

Agora, como na Proposição 5.1.2, escolhendo uma subseqüência $\widehat{\Gamma}_{1, n_{i}}$ convergente a $\widehat{\Gamma}^{*}$ obtemos que $\widehat{\Gamma}^{*} \subset \widehat{\Gamma}_{1}, L \in \widehat{\Gamma}^{*}$. e por construção $\widehat{\Gamma}^{*} \subset \widehat{\Gamma}_{2 a, \text { down }}$, e do Lema 5.1.2 concluimos que 
$\left[\left(\Gamma_{1} \cap V_{a}^{-}\right)\right] \subset \Gamma_{2 a, \text { down }}$ o que contradiz o fato de ter $\left[\Gamma_{1} \cap V_{a}^{-}\right] \subset \Gamma_{2 a, \text { up }}$ e prova a afirmação.

Da afirmação 5.1.2, escolhemos $c \leq b$ satisfazendo o anterior, então temos $\Gamma_{2 c, \text { down }} \subset \Gamma_{2 b, \text { down }}$, logo temos que $\Gamma_{1} \cap \Gamma_{2 c, \text { down }}=\left(\right.$ pois $\Gamma_{2 c, \text { down }} \subset \Gamma_{2 a, \text { down }}$, e $\left(\Gamma_{1} \cap V_{c}^{-}\right) \cap \Gamma_{2 a, \text { down }}=\emptyset$, logo da definição dos conjuntos $\Gamma_{2 c, \text { down }}$ e $\Gamma_{2 a, \text { down }}$ implica o anterior).

Então, existe um arco continuo simples $\beta_{0}$, que conecta um ponto de $\Gamma_{2}$ a algum ponto de $(-\infty, c) \times\{0\}$. Note que podemos escolher $\beta_{0}$ de modo que $\beta_{0} \cap \Gamma_{2}$ é um extremo de $\beta_{0}$, denotado $m_{0}$, e $\beta_{0} \backslash\left\{m_{0}\right\} \subset \Gamma_{2 c, \text { down }}$, obtendo, pelo trabalhado acima, que $\beta_{0} \cap \Gamma_{1}=\emptyset$.

Tomando o conjunto conexo e fechado $\left(\beta_{0} \cup \Gamma_{2} \cup \beta_{1}\right)$, temos que ele contém pontos de $\mathbf{R} \times\{0\}$ e de $\mathbf{R} \times\{1\}$ e, além disso, por construção

$$
\left(\beta_{0} \cup \Gamma_{2} \cup \beta_{1}\right) \cap\left(\alpha \cup \Gamma_{1}\right)=\emptyset
$$

E obtemos assim a mesma contradição que no Lema 5.1.2, e issa contradição prova o Lema 5.1.3.

Então, notemos que, até agora, provamos que se $V_{a}$ intercepta $\Gamma_{1}$ e $\Gamma_{2}$, sendo estas componentes conexas de $D$, temos $\Gamma_{2} \prec_{a} \Gamma_{1}$ ou $\Gamma_{1} \prec_{a} \Gamma_{2}$. Vamos provar no próximo lema, que essa ordem independe da escolha de $a$.

Lema 5.1.4 : Sejam $\Gamma_{1}, \Gamma_{2}$ duas componentes conexas diferentes de $D$, e sejam a, $b \leq 0$ tais que $\Gamma_{1}$ e $\Gamma_{2}$ interceptam $V_{a}$ e $V_{b}$. Então temos o seguinte

$$
\begin{aligned}
& \Gamma_{1} \prec_{a} \Gamma_{2} \Leftrightarrow \Gamma_{1} \prec_{b} \Gamma_{2} \\
& \Gamma_{2} \prec_{a} \Gamma_{1} \Leftrightarrow \Gamma_{2} \prec_{b} \Gamma_{1}
\end{aligned}
$$

Demonstração: Suponha que $b<a$ e $\Gamma_{2} \prec{ }_{a} \Gamma_{1}$, por definição $\left[\Gamma_{2} \cap V_{a}^{-}\right] \subset \Gamma_{1 a, \text { down }}$. Logo, da afirmação 5.1.2, o conjunto $\left(\Gamma_{2} \cap V_{a}^{-}\right) \cap \Gamma_{1 a, u p}$ é limitado. Como $\left(\Gamma_{2} \cap V_{b}^{-}\right) \subset\left(\Gamma_{2} \cap V_{a}^{-}\right)$e $\Gamma_{1 b, u p} \subset \Gamma_{1 a, u p}$ temos que $\left(\Gamma_{2} \cap V_{b}^{-}\right) \cap \Gamma_{1 b \text {,up }}$ é um conjunto limitado, como o qual obtemos que $\left[\Gamma_{2} \cap V_{b}^{-}\right] \subset \Gamma_{1 b \text {,down }}$ que por definição implica $\Gamma_{2} \prec_{b} \Gamma_{1}$.

Se $\Gamma_{2} \prec_{b} \Gamma_{1}$, por definição $\left[\Gamma_{2} \cap V_{b}^{-}\right] \subset \Gamma_{1 b \text {,down }}$, como $b<a$, temos $\left[\Gamma_{2} \cap V_{b}^{-}\right] \subset\left[\Gamma_{2} \cap V_{a}^{-}\right]$ (isso olhado como conjuntos) e $\Gamma_{1 b, \text { down }} \subset \Gamma_{1 a \text {,down }}$, logo, claramente temos $\left[\Gamma_{2} \cap V_{a}^{-}\right] \subset \Gamma_{1 a, \text { down }}$, pois alguma componente conexa ilimitada de $\left[\Gamma_{2} \cap V_{b}^{-}\right]$tem interseção não vazia como $\Gamma_{1 a \text {,down }}$, e issa componente forma parte de uma componente conexa ilimitada de $\left[\Gamma_{2} \cap V_{a}^{-}\right]$, logo o Lema 5.1.2 implica o afirmado, obtendo que $\Gamma_{2} \prec_{a} \Gamma_{1}$. Provamos assim o Lema, pois a outra equivalencia é provada da mesma forma.

Para concluir com as propriedades de ordem, precisamos provar o seguinte Lema.

Lema 5.1.5 : Se $\Gamma_{1}, \Gamma_{2}, \Gamma_{3}$ são componentes conexas de $D, a \leq 0$ tal que $V_{a}$ intercepta $\Gamma_{1}, \Gamma_{2}$, $\Gamma_{3}$, e elas satisfazem que $\Gamma_{1} \prec_{a} \Gamma_{2}$ e $\Gamma_{2} \prec_{a} \Gamma_{3}$, então $\Gamma_{1} \prec_{a} \Gamma_{3}$.

Demonstração: Por a hipóteses do lema, temos que $\left[\Gamma_{1} \cap V_{a}^{-}\right] \subset \Gamma_{2 a, \text { down }}$ e $\left[\Gamma_{2} \cap V_{a}^{-}\right] \subset \Gamma_{3 a, \text { down }}$. Notemos que do Lema 5.1.2 e da demonstração do Lema 5.1.3, obtemos que, se $\Theta$ e $\Lambda$ são duas componentes conexas de $D$ interceptando $V_{a}$, o fato de $\left[\Theta \cap V_{a}^{-}\right] \subset \Lambda_{a, \text { down }}$, implica que $\left[\Lambda \cap V_{a}^{-}\right] \subset$ $\Theta_{a, u p}$. Desta última observação, obtemos $\left[\Gamma_{2} \cap V_{a}^{-}\right] \subset \Gamma_{1 a, u p}$, e $\left[\Gamma_{3} \cap V_{a}^{-}\right] \subset \Gamma_{2 a, u p}$. Logo, pela afirmação 5.1 .2 , tomamos $b \leq a$ tal que as seguintes afirmações são satisfeitas:

$$
\begin{gathered}
\Gamma_{3} \cap V_{b}^{-} \subset \Gamma_{2 b, \text { up }} \\
\Gamma_{1} \cap V_{b}^{-} \subset \Gamma_{2 b, \text { down }} \\
\Gamma_{2} \cap V_{b}^{-} \subset \Gamma_{3 b \text {,down }}
\end{gathered}
$$

Agora suponha que $\Gamma_{2 b, \text { down }}$ não esteja contida em $\Gamma_{3 b \text {,down }}$, logo existe um arco continuo simples $\alpha \subset \Gamma_{2 b \text {,down }}$ conectando um ponto de $(-\infty, b) \times\{0\}$ com um ponto $p \notin \Gamma_{3 b \text {,down }}$, como $(-\infty, b) \times\{0\} \subset \Gamma_{3 b \text {,down }}$ temos que $\alpha$ intercepta $\partial \Gamma_{3 b \text {,down }} \subset \Gamma_{3}$, logo $\alpha$ intercepta $\Gamma_{3}$, e dado que $\alpha \subset(-\infty, b) \times[0,1]$ (pois $\left.\alpha \subset \Gamma_{2 b, \text { down }}\right)$, obtemos $\alpha \cap\left(\Gamma_{3} \cap V_{b}^{-}\right) \neq \emptyset$. Mas da primeira linha das inclusões de acima, obtemos que $\alpha \cap \Gamma_{2 b, u p}$, logo obtemos que $\alpha \subset \Gamma_{2 b \text {,down }}$ e $\alpha \cap \Gamma_{2 b, u p} \neq \emptyset$, o qual

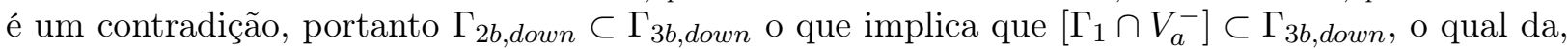


por definição que $\Gamma_{1} \prec \Gamma_{3}$ que era o que queríamos provar.

Provado este último lema, estamos em condições de definir uma relação de ordem sobre as componentes conexas de $D$. Mas notemos a hipótese de que os conjuntos eram componentes conexas de $D$ foi para assegurar que eles eram disjuntos, ilimitados a esquerda, contidos em $V^{-}$e como complementar conexo, logo, todo trabalho feito até aqui pode ser aplicado diretamente sobre as hipóteses uma familia de conjuntos fechados, disjuntos, conexos, ilimitados a esquerda, contidos em $V^{-}$e com complementares conexos. A próxima definição é mais geral, e aplica-se como caso particular sobre as componentes conexas do conjunto $D$.

Definição: Sejam $\Theta$ e $\Lambda$ conjuntos fechados disjuntos, conexos, ilimitados a esquerda, contidos em $V^{-}$com complementares conexos. Dizemos que

$$
\Theta \prec \Lambda
$$

se para qualquer, e portanto para todo $a \leq 0$ satisfazendo que $V_{a}$ intercepta $\Theta$ e $\Lambda$ se cumpre que

$$
\left[\Theta \cap V_{a}^{-}\right] \subset \Lambda_{a, \text { down }}
$$

Como já falamos acima, a definição anterior se aplica no caso particular das componentes conexas de $D$, e o trabalho até o final da seção, será sobre as componentes conexas de $D$, para provar que, sobre elas, $\widetilde{f}$ preserva a ordem definida. Para isso vamos provar mas alguns resultados, e usar a definição dada acima. O motivo para usar a definição anterior é porque, sendo $\Gamma_{1}$ e $\Gamma_{2}$ componentes conexas de $D$, pode acontecer que $\widetilde{f}\left(\Gamma_{1}\right)$ e $\widetilde{f}\left(\Gamma_{2}\right)$ estejam na mesma componente conexa, mas elas continuarão satisfazendo a definição anterior, e portanto estarão ordenadas, isto é, provaremos que $\widetilde{f}$ preserva a ordem, ou seja se $\Gamma_{2} \prec \Gamma_{1}$ então $\widetilde{f}\left(\Gamma_{2}\right) \prec \widetilde{f}\left(\Gamma_{1}\right)$.

Definição: Denotamos por $\Gamma^{+}$à componente conexa de $D$ que contém $\widetilde{f}(\Gamma)$, isto é, $\widetilde{f}(\Gamma) \subset \Gamma$.

Lema 5.1.6 : Sejam $\Gamma_{1}$ e $\Gamma_{2}$ componentes conexas de D, e suponha $\Gamma_{1} \prec \Gamma_{2}$ então $\tilde{f}\left(\Gamma_{1}\right) \prec \widetilde{f}\left(\Gamma_{2}\right)$, e, se $\Gamma_{1}^{+} \neq \Gamma_{2}^{+}$, então $\Gamma_{1}^{+} \prec \Gamma_{2}^{+}$

Demonstração: Suponha que $\Gamma_{2}^{+} \prec \Gamma_{1}^{+}$. Como $\Gamma_{1} \prec \Gamma_{2}$, para qualquer $a \leq 0$ tal que $\Gamma_{1}$ e $\Gamma_{2}$, agora da prova do Lema 5.1.3 e o anterior temos que $\left[\Gamma_{2} \cap V_{a}^{-}\right] \subset \Gamma_{1 a, u p}$.

Pelo já provado, podemos escolher $b<a<0$ suficientemente pequeno tal que

$$
\begin{gathered}
\Gamma_{1} \cap V_{b}^{-} \subset \Gamma_{2 b, \text { down }} \\
\Gamma_{2} \cap V_{b}^{-} \subset \Gamma_{1 b, \text { up }} \\
\Gamma_{2}^{+} \cap V_{b}^{-} \subset \Gamma_{1 b, \text { down }}^{+}
\end{gathered}
$$

Agora tomemos $c<b$ suficientemente pequeno tal que $\widetilde{f}^{ \pm}\left(V_{c}\right) \cap V_{b}=\emptyset$, e que como antes, satisfaça que

$$
\begin{gathered}
\Gamma_{1} \cap V_{c}^{-} \subset \Gamma_{2 c, \text { down }} \\
\Gamma_{2} \cap V_{c}^{-} \subset \Gamma_{1 c, \text { up }} \\
\Gamma_{2}^{+} \cap V_{c}^{-} \subset \Gamma_{1 c, \text { down }}^{+}
\end{gathered}
$$

Então existe um arco continuo simples $\alpha \subset \Gamma_{1 c, \text { down }}^{+}$que conecta um ponto de $(-\infty, c) \times\{0\}$ a um ponto $P \in \widetilde{f}\left(\Gamma_{2}\right) \subset \Gamma_{2}^{+}$. Da escolha do $c, \widetilde{f}^{-1}(\alpha) \subset V_{b}^{-}$e conecta um ponto de $(-\infty, b) \times\{0\}$ a $\widetilde{f}^{-1}(P) \in \Gamma_{2}$.

Como $\alpha \subset \Gamma_{1 c, \text { down }}^{+}, \alpha \cap \Gamma_{1}^{+}=\emptyset, \operatorname{logo} \tilde{f}^{-1}(\alpha) \cap \Gamma_{1}=\emptyset$. Pelas hipóteses sobre $c$, temos que $\alpha$ contém um ponto de $(-\infty, b) \times\{0\}$ e $\widetilde{f}^{-1}(\alpha) \cap(-\infty, b) \times\{0\} \neq \emptyset$, o qual implica que $\widetilde{f}^{-1}(\alpha) \cap \Gamma_{1 b, \text { down }} \neq \emptyset$ e $\widetilde{f}^{-1}(\alpha) \subset V_{b}^{-}$, sumado a que $\widetilde{f}^{-1}(\alpha) \cap \Gamma_{1}=\emptyset$ obtemos, por argumento de conexidade maximal (e o fato de que as componenetes conexas por curvas e as componentes conexas são iguais) que 
$\widetilde{f}^{-1}(\alpha) \subset \Gamma_{1 b, \text { down }}$

Mas a escolha de $\alpha$ e todo o anterior implica que $\Gamma_{2} \cap \Gamma_{1 b \text {,down }} \neq \emptyset$, contradizendo a escolha do $b$ e o fato de ter $\Gamma_{2} \cap V_{b}^{-} \subset \Gamma_{1 b, u p}$. Esta contradição chego de supor que, tendo $\Gamma_{1} \prec \Gamma_{2}$ se satisfaz $\tilde{f}\left(\Gamma_{2}\right) \prec \widetilde{f}\left(\Gamma_{1}\right)$, logo provamos que se $\Gamma_{1} \prec \Gamma_{2}$ então $\tilde{f}\left(\Gamma_{1}\right) \prec \widetilde{f}\left(\Gamma_{2}\right)$. E claramente, se $\Gamma_{1}^{+} \neq \Gamma_{2}^{+}$, a prova anterior mostra que $\Gamma_{1}^{+} \prec \Gamma_{2}^{+}$concluindo assim a prova do Lema.

Nos casos em que $D=B^{-}$, ou $D=\omega\left(B^{-}\right)$, pode-se mostrar que $\widetilde{f}^{-1}$ também preserva a ordem. No caso $D=\omega\left(B^{-}\right)$é trivial, pois $\omega\left(B^{-}\right)$é $\widetilde{f}$-invariante, então em particular, $\widetilde{f}^{-1}\left(\omega\left(B^{-}\right)\right) \subset \omega\left(B^{-}\right)$ e aplicamos diretamente o Lema anterior.

Quando $D=B^{-}$, se $\Gamma$ é uma componente conexa de $B^{-}, \widetilde{f}^{-1}(\Gamma)=\Gamma^{-}$é também um conjunto fechado, conexo, ilimitado a esquerda e limitado a direita, mas é posível que não esteja contido em $B^{-}$, ou seja que $\Gamma^{-} \cap(0,+\infty) \times[0,1] \neq \emptyset$. Então temos duas possibilidades:

- $\tilde{f}^{-1}(\Gamma) \cap(0,+\infty) \times[0,1]=\emptyset$. Como $\tilde{f}\left(\tilde{f}^{-1}(\Gamma)\right)=\Gamma^{-}$, existe uma componente conexa de $B^{-}, \Gamma^{-}$, que satisfaz $\Gamma^{-} \supset \widetilde{f}^{-1}(\Gamma)$ e, claramente $\widetilde{f}\left(\Gamma^{-}\right) \cap \Gamma \neq \emptyset$, o seja $\widetilde{f}\left(\Gamma^{-}\right) \subset \Gamma$, portanto $\Gamma^{-}=\widetilde{f}^{-1}(\Gamma)$.

- $\widetilde{f}^{-1}(\Gamma) \cap(0,+\infty) \times[0,1] \neq \emptyset$. Como $\left[\tilde{f}^{-1}(\Gamma) \cap V^{-}\right]$tem, pelo menos uma componente conexa, $\Gamma^{*}$, temos que $\widetilde{f}\left(\Gamma^{*}\right) \subset \Gamma \subset B^{-}$, então existe uma componente conexa de $B^{-}, \Gamma^{-}$, que contém $\Gamma^{*}$ e $\widetilde{f}\left(\Gamma^{-}\right) \subset \widetilde{\sim}$. Claramente $\Gamma^{-}$não é necessariamente única, pois a componentes conexa ilimitada de $\widetilde{f}^{-1}(\Gamma) \cap V^{-}$não precisa ser única.

Mas, em ambos os casos, temos que:

Lema 5.1.7 : Sejam $\Gamma_{1}, \Gamma_{2}$ componentes conexas de D, e suponha que $\Gamma_{1} \prec \Gamma_{2}$, então, para qualquer escolha de $\Gamma_{1}^{-}$e $\Gamma_{2}^{-}$, temos $\Gamma_{1}^{-} \prec \Gamma_{2}^{-}$.

Demonstração: Usando o Lema anterior, como $\tilde{f}$ preserva ordem, se $\Gamma_{1} \neq \Gamma_{2}$, e supondo $\Gamma_{2}^{-} \prec \Gamma_{1}^{-}$, obtemos que $\Gamma_{2} \prec \Gamma_{1}$, contradizendo nossa hipótese, logo só pode acontecer que $\Gamma_{1}^{-} \prec \Gamma_{2}^{-}$, como queriamos provar.

Provados todos estes resultados, vamos analisar agora que acontece quando consideramos uma componente conexa de $D, \Gamma$, e sua projeção sobre $A, p(\Gamma)$. Notemos que podem acontecer duas situações:

- Que $\left.p\right|_{\Gamma}$ não seja injetora, isto é, $p\left(\widetilde{z}_{1}\right)=p\left(\widetilde{z}_{2}\right)$ para uma certa escolha de $\widetilde{z}_{1}, \widetilde{z}_{2} \in \Gamma, \widetilde{z}_{1} \neq \widetilde{z}_{2}$.

- Que $\left.p\right|_{\Gamma}$ seja injetora, isto é, $p\left(\widetilde{z}_{1}\right) \neq p\left(\widetilde{z}_{2}\right)$, para qualquer $\widetilde{z}_{1}, \widetilde{z}_{2} \in \Gamma$.

Nas próximas seções, vamos analisar os dois casos por separado.

\section{$5.2 \Gamma \subset D$, componente conexa, e $\left.p\right|_{\Gamma}$ não injetora.}

Então, temos que existe $\widetilde{z} \in \widetilde{A}$, e um inteiro $s>0$ tal que $\widetilde{z}, \widetilde{z}+(s, 0) \in \Gamma$, $\log$ o $\Gamma \cap \Gamma+(s, 0) \neq \emptyset$. Também é claro que $\Gamma-(s, 0) \subset D$, pois é um conjunto conexo, ilimitado a esquerda e contido em $V^{-}$. Todo o anterior implica que

$$
\Gamma-(s, 0) \subset \Gamma
$$

pois $\Gamma$ é uma componente conexa de $D$.

Suponha que $\Gamma-(1,0)$ não estivese contida em $\Gamma$, como $\Gamma-(1,0) \subset D$, temos, por conexidade maximal, $\Gamma-(1,0) \cap \Gamma=\emptyset$. Agora, $\Gamma-(1,0)$ é conexo, fechado, não intercepta $\Gamma$ e também, 
não intercepta $V_{m_{\Gamma}}=\left\{m_{\Gamma}\right\} \times[0,1]$ (ver definição de $m_{\Gamma}$ na seção anterior), logo, o Lema 5.1.2 implica, dado que por definição dos conjuntos $\left[\Gamma-(1,0) \cap V_{m_{\Gamma}}^{-}\right]=\Gamma-(1,0)$, que uma, e só uma das seguintes possibilidades é satisfeita:

$$
\Gamma-(1,0) \subset \Gamma_{\text {down }} \text { ou } \Gamma-(1,0) \subset \Gamma_{\text {up }} .
$$

Sem perda de generalidade, suponha $\Gamma-(1,0) \subset \Gamma_{u p}$ (a outra possibilidade é análoga). Então

Proposição 5.2.1 : Se $\Gamma-(1,0) \subset \Gamma_{u p}$, então $\Gamma-(i, 0) \subset \Gamma_{\text {up }}$, para todo inteiro $i>1$.

Demonstração: Suponha que exista $s_{0}>1$, onde eu posso supor que $s_{0}$ é o primeiro inteiro positivo para o qual acontece que $\Gamma-\left(s_{0}, 0\right) \subset \Gamma$, logo temos que $\Gamma, \Gamma-(1,0), \ldots, \Gamma-\left(s_{0}-1,0\right)$ são todos disjuntos (caso contrário, obtemos que, ou $s_{0}$ não existe, ou $s_{0}$ não é o primer inteiro para qual acontece o anterior).

Como $\Gamma-(1,0) \subset \Gamma_{u p}$ (o qual implica, pelo feito na demonstração do Lema 5.1.3) que $\Gamma \prec$ $\Gamma-(1,0)$ dando como resultado que $\Gamma-(s, 0) \cap \Gamma-(s+1,0)=\emptyset$ e $\Gamma-(s, 0) \prec \Gamma-(s+1,0)$ para todo inteiro $s>0$ (o último acontece, pois de não ser certo para algum $s_{1}$, tomando um $V_{a}$ apropriado, obtemos trasladando $V_{a}$ por $s_{1}$, que $\Gamma-(1,0) \prec \Gamma$, o qual é uma contradição).

Então, em particular, usando o fato de $\prec$ ser uma relação de ordem, temos:

1. $\Gamma \prec \Gamma-(1,0) \prec \Gamma-(2,0) \prec \ldots \prec \Gamma-\left(s_{0}-1,0\right)$

2. $\Gamma-\left(s_{0}-1,0\right) \prec \Gamma-\left(s_{0}, 0\right)$

Agora, por nossa suposição, temos $\Gamma-\left(s_{0}, 0\right) \subset \Gamma$ e $\Gamma \cap \Gamma-\left(s_{0}-1,0\right)=\emptyset$ temos, de 2 acima, que para qualquer $a$ tal que $V_{a} \cap \Gamma-\left(s_{0}, 0\right) \neq \emptyset$, também se da que $V_{a} \cap \Gamma \neq \emptyset$ e $\left(\Gamma-\left(s_{0}, 0\right)\right)_{a, \text { down }} \subset \Gamma_{a, \text { down }}$, logo, todo o anterior implica que $\Gamma-\left(s_{0}-1,0\right) \prec \Gamma$, e isso contradiz 1 de acima.

A contradição chegou de supor que $\Gamma \cap \Gamma-\left(s_{0}, 0\right) \neq \emptyset$ para algum $s_{0}>0, \log 0 \Gamma \cap \Gamma-(i, 0)=\emptyset$ para todo inteiro $i>0 \mathrm{e}$

$$
\Gamma \prec \Gamma-(1,0) \prec \Gamma-(2,0) \prec \ldots \prec \Gamma-(i, 0)
$$

Agora se $a \in \mathbf{R}$ é tal que $\Gamma$ e $\Gamma-(i, 0)$ interceptam $V_{a}$ então $\left[\Gamma \cap V_{a}^{-}\right] \subset(\Gamma-(i, 0))_{a, \text { down }}$ se e só se $\left[\Gamma-(i, 0) \cap V_{a}^{-}\right] \subset \Gamma_{a, u p}$, como claramente $\Gamma_{a, u p} \subset \Gamma_{u p}$, temos que $\Gamma-(i, 0) \cap \Gamma_{u p} \neq \emptyset$, e como por construção temos $\Gamma-(i, 0) \cap V_{m_{\Gamma}}=\emptyset$, do Lema 5.1.2 obtemos $\Gamma-(i, 0) \subset \Gamma_{u p}$ como afirmamos.

Fazendo o mesmo raciocínio, obtemos o mesmo resultado para $\Gamma-(1,0) \subset \Gamma_{\text {down }}$. Então, dado que pelo fato de $\left.p\right|_{\Gamma}$ ser não injetora, $\Gamma-\left(s_{0}, 0\right) \subset \Gamma$ para algum $s_{0}>0$, por o contrapositivo da proposição anterior obtemos que

$$
\Gamma-(1,0) \subset \Gamma
$$

Para finalizar, se $\left.p\right|_{\Gamma}$ não é injetora, dizemos que $\Gamma$ é uma componente não injetora.

\section{3 $\Gamma \subset D$, componente conexa, e $\left.p\right|_{\Gamma}$ injetora.}

O fato de $\left.p\right|_{\Gamma}$ ser injetora, implica que $\Gamma \cap \Gamma+(s, 0)=\emptyset$, para todo inteiro $s \neq 0$. Em particular $\Gamma \cap \Gamma-(1,0)=\emptyset$ (lembrar também que $\Gamma \cap \mathbf{R} \times\{i\}=\emptyset$ para $i=0,1)$. Usando esta idéia vamos dar a seguinte definição.

Definição: Dizemos que $\Gamma$ é uma componente inferior se $\Gamma \prec \Gamma-(1,0)$. E dizemos que $\Gamma$ é uma componente superior se $\Gamma-(1,0) \prec \Gamma$.

Então temos o seguinte

Lema 5.3.1 : Se $\Gamma \subset D$ é uma componente conexa inferior, então 


$$
d(\Gamma ; \mathbf{R} \times\{1\})>0
$$

$e$, analogamente, se $\Gamma \subset D$ é uma componente superior então

$$
d(\Gamma ; \mathbf{R} \times\{0\})>0
$$

Demonstração: Vamos provar para o caso em que $\Gamma$ é uma componente inferior, o outro caso é análogo.

Então, para qualquer $\widetilde{x}<m_{\Gamma}$, se consideramos o segmento $\{\widetilde{x}\} \times\left[0, \widetilde{y}^{*}\right]$, onde

$$
\widetilde{y}^{*}=\widetilde{y}^{*}(\widetilde{x})=\sup \{\widetilde{y} \in(0,1): \Gamma \cap\{\widetilde{x}\} \cap[0, \widetilde{y}]=\emptyset\}
$$

Notando que o ponto $\left(\widetilde{x}, \widetilde{y}^{*}\right) \in \Gamma$, como $\Gamma-(1,0) \subset \Gamma_{\text {up }}$ temos que

$$
\Gamma-(1,0) \cap\{\widetilde{x}\} \times\left[0, \widetilde{y}^{*}\right]=\emptyset
$$

Agora tomemos o ponto $\left(m_{\Gamma}-1, \widetilde{y}_{\Gamma}\right) \in \Gamma-(1,0)$ e um arco continuo simples $\gamma \subset \mathbf{R} \times(0,1)$, tal que:

1. $\gamma \cap(\Gamma-(1,0))=\left(m_{\Gamma}-1, \widetilde{y}_{\Gamma}\right)$

2. $\gamma \cap \Gamma=\emptyset$

3. Os pontos extremos de $\gamma$ são $\left(m_{\Gamma}-1, \widetilde{y}_{\Gamma}\right)$ e $\left(m_{\Gamma}+1,0.5\right)$

4. $\gamma \cap\left\{m_{\Gamma}+1\right\} \times[0,1]=\left(m_{\Gamma}+1,0.5\right)$

Dado que $\Gamma-(1,0) \subset \Gamma_{u p}$ e $(\Gamma \cup \Gamma-(1,0))^{c}$ é conexo, é posível escolher $\gamma$ satisfazendo o anterior.

Agora também notemos que, se tomamos o conjunto $\Gamma-(1,0) \cup \gamma \cup\left\{m_{\Gamma}+1\right\} \times[0,1]$, seu complementar possui, por contrução e por ser $\left.p\right|_{\Gamma}$ injetora, exactamente duas componentes conexas ilimitadas a esquerda em $\left(-\infty, m_{\Gamma}+1\right) \times[0,1]$, uma contendo $\left(-\infty, m_{\Gamma}\right) \times\{0\}$, denotada por $(\Gamma-(1,0))_{\text {down }}$ e outra contendo $\left(-\infty, m_{\Gamma}\right) \times\{1\}$, chamada $(\Gamma-(1,0))_{\text {up }}$. Dado que a escolha do ponto $\left(m_{\Gamma}, \widetilde{y}_{\Gamma}\right)$ foi arbitraria, a construção feita acima pode não ser única, mas qualquera seja a escolha do ponto, afirmamos que $\Gamma \subset(\Gamma-(1,0))_{\text {down }}$. O anterior é certo já que, considerando

$$
\widetilde{x}<\min \left\{m_{\Gamma} ; \min \{\widetilde{x} \in \mathbf{R}:(\widetilde{x}, \widetilde{y}) \in \gamma, \text { para algum } \widetilde{y} \in(0,1)\}\right\}-10
$$

Temos que, tomando $\widetilde{x}$ como acima, o segmento $V_{\widetilde{x}}$ não intercepta $\gamma$ (pela escolha), e também temos, pelo fato de estar $\Gamma-(1,0) \subset \Gamma_{u p}$ e ter $\Gamma \cap \gamma=\emptyset$ que, escolhendo $\widetilde{y}^{*}$ como antes, o segmento $\{\widetilde{x}\} \times\left[0, \widetilde{y}^{*}\right]$ não intercepta $\Gamma-(1,0) \cup \gamma \cup\left\{m_{\Gamma}+1\right\} \times[0,1]$, e como $\left(\widetilde{x}, \widetilde{y}^{*}\right) \in \Gamma$ obtemos $\Gamma \cap(\Gamma-(1,0))_{\text {down }} \neq \emptyset$, o que implica

$$
\Gamma \subset(\Gamma-(1,0))_{\text {down }}
$$

Agora, suponha que $d(\Gamma, \mathbf{R} \times\{1\})=0$ (note que isso implica que $\Gamma$ vai-se aproximar a $\mathbf{R} \times\{1\}$ quando $\widetilde{z} \in \Gamma$ satisfaçam que $p_{1}(\widetilde{z}) \rightarrow-\infty$, isto pela escolha de $\gamma$ e o fato de $\mathbf{R} \times\{1\}$ e $\Gamma$ ser fechados), como $\Gamma \cap \mathbf{R} \times\{1\}=\emptyset$ temos que para qualquer $M$ que satisfaz

$$
M \leq M_{0}=\min \left\{m_{\Gamma}-10 ; \min \{\widetilde{x} \in \mathbf{R}:(\widetilde{x}, \widetilde{y}) \in \gamma, \text { para algum } \widetilde{y} \in(0,1)\}\right\}-10
$$

existe um $\epsilon>0$ tal que, se $\widetilde{z} \in \Gamma \cap \mathbf{R} \times[1-\epsilon, 1]$, então, pelo falado antes, $p_{1}(\widetilde{z})<M$.

Então, tomemos $M_{0}$ e $\epsilon>0$ como acima, e escolhamos $\widetilde{z}_{0} \in \Gamma \cap \mathbf{R} \times[1-\epsilon, 1]$ tal que

$$
p_{1}\left(\widetilde{z}_{0}\right) \geq p_{1}(\widetilde{z}) \quad \forall \widetilde{z} \in \mathbf{R} \times[1-\epsilon, 1]
$$

$\mathrm{e}$

$$
d\left(\widetilde{z}_{0} ; \mathbf{R} \times\{1\}\right)<d(\widetilde{z} ; \mathbf{R} \times\{1\}) \quad \forall \widetilde{z} \in \Gamma \cap\left\{p_{1}\left(\widetilde{z}_{0}\right)\right\} \times[1-\epsilon, 1] \operatorname{com} \widetilde{z} \neq \widetilde{z}_{0}
$$


Basicamente, o que fizimos foi, considerar a partir de $\left\{m_{\Gamma}\right\} \times[0,1]$, o ponto $\widetilde{z}_{0} \in \Gamma$ com o maior valor na primeira componente, que esteja contido em $\mathbf{R} \times[1-\epsilon, 1]$, e que também tenha o maior valor na segunda componente, quando considero os elementos que estão em $\Gamma \cap\left\{p_{1}\left(\widetilde{z}_{0}\right\} \times[1-\epsilon, 1]\right.$.

Agora, considere o segmento vertical fechado $l$, contido em $\mathbf{R} \times[1-\epsilon, 1]$, começando em $\widetilde{z}_{0}$ e acabando em $\mathbf{R} \times\{1\}$. Por construção de $l, l \cap \Gamma=\widetilde{z}_{0}$, e (também por contrução de $l$ e $\gamma$ ) $l \cap\left(\gamma \cup\left\{m_{\Gamma}+1\right\} \times[0,1]=\emptyset\right.$. Como $\Gamma \subset(\Gamma-(1,0))_{\text {down }}$, pelo Lema 5.1.2 temos $\Gamma-(1,0) \subset \Gamma_{\text {up }}$, implicando que $l \cap(\Gamma-(1,0)) \neq \emptyset$ (pela escolha de $\left.M_{0}\right)$.

Todo o anterior mostra que existe $\widetilde{z}_{1} \in l \cap \Gamma-(1,0)$, o que implica que

$$
\widetilde{z}_{1}+(1,0) \in l+(1,0) \cap \Gamma
$$

Mas isso contradiz a escolha do $\widetilde{z}_{0}$, pois ele era o ponto de $\Gamma \cap \mathbf{R} \times[1-\epsilon, 1]$ com maior valor na sua primeira coordenada. Esta contradição foi obtida supondo que $d(\Gamma ; \mathbf{R} \times\{1\})=0$, logo, temos provado lema.

\subsection{Demonstração do Teorema 4}

Agora, provados os resultados preliminares, vamos dar uma demonstração da densidade de $p\left(B^{-}\right)$. Aqui vamos usar certos fatos já provados sobre o comportamento de $p(D)$.

Supondo que $\overline{p\left(B^{-}\right)} \neq A$, vamos tomar uma componente conexa $\Gamma_{1}$ que, pelo provado anteriormente, podemos supor que satisfaz $\overline{p\left(\Gamma_{1}\right)} \supset \mathbf{S}^{1} \times\{0\}$. Feito isso, vamos provar, primeiro que ou $\Gamma_{1}^{+} \subset \Gamma_{1}$ ou $\Gamma_{1}^{+} \prec \Gamma_{1}$ e, separando por casos ( $\Gamma_{1}$ uma componente injetora, ou $\Gamma_{1}$ uma componente não injetora), vamos provar que nenhuma das duas possibilidades pode acontecer, o qual é uma contradição que provara que $p\left(B^{-}\right)$é denso em $A$.

Começamos assumindo que $\overline{p\left(B^{-}\right)} \neq A$. Dos fatos provados na secção 4.6 para o comportamento de $p(D)$, temos que existe uma componente conexa $\Gamma$ de $B^{-}$que satisfaz $p(\Gamma) \subset \gamma_{E}^{-}, \overline{p(\Gamma)} \supset \mathbf{S}^{1} \times\{0\}$ (podemos supor isso sem perda de generalidade, com a outra possibilidade trabalha-se de maneira análoga), sendo $\gamma_{E}$ a curva homtopicamente não trivial, fechada, simples em $A$, com $\gamma_{E} \subset{\overline{p\left(B^{-}\right)}}^{c}$.

Agora vamos fazer a mesma construção da demonstração do Teorema 2, só trocando $\omega\left(B^{-}\right)$por $B^{-}-$

Seja $\sigma>0$ tal que $p_{1}(\widetilde{f}(\widetilde{x}, 0))>\widetilde{x}+2 \sigma$, para todo $\widetilde{x} \in \mathbf{R}$. Pela continuidade de $\widetilde{f}$, podemos escolher $\epsilon>0$ suficientemente pequeno tal que

- $\mathbf{S}^{1} \times[0, \epsilon] \subset \gamma_{E}^{-}$

- $p_{1} \circ \widetilde{f}(\widetilde{x}, \widetilde{y})>\widetilde{x}+\sigma \quad \forall(\widetilde{x}, \widetilde{y}) \in \mathbf{R} \times[0, \epsilon]$

Como $\mathbf{S}^{1} \times\{0\} \subset \overline{p(\Gamma)}$, existe um numero real $a<0$ satisfazendo

$$
\Gamma \cap\{a\} \times[0, \epsilon] \neq \emptyset
$$

Do fato de $B^{-}$ser fechado, existe $0<\delta \leq \epsilon$ tal que o ponto $(a, \delta) \in B^{-}$e tal que, para todo $\widetilde{y}$, $0 \leq \widetilde{y} \leq \delta,(a, \widetilde{y}) \notin B^{-}$, ou seja, $(a, \delta)$ é o menor ponto de $B^{-}$no segmento $\{a\} \times[0, \epsilon]$. Vamos denotar por $v$ ao segmento $\{a\} \times[0, \delta)$ (note que $B^{-} \cap v=\emptyset$ ).

Tomemos $\Gamma_{1}$ a componente conexa de $B^{-}$que contém $(a, \delta)$. Como $p\left(\Gamma_{1}\right) \cap \gamma_{E}^{-} \neq \emptyset$, claramente, pelo provado antes, isso implica que

$$
\begin{gathered}
d\left(p\left(\Gamma_{1}\right) ; \mathbf{S}^{1} \times\{1\}\right)>0 \\
d\left(\Gamma_{1} ; \mathbf{R} \times\{1\}\right)>0
\end{gathered}
$$

Afirmamos que $\Gamma_{1}^{+}$não está acima de $\Gamma_{1}$. Para mostrar que significa isso, vamos provar primeiro o seguinte

Proposição 5.4.1: $\Gamma_{1}^{+} \cap v=\emptyset$ 
Demonstração: $\Gamma_{1}^{+}$é uma componente conexa de $B^{-}$, pelo falado acima, $B^{-} \cap v=\emptyset$, logo $\Gamma_{1}^{+} \cap v=\emptyset$.

Proposição 5.4.2 : Se $\Gamma_{1} \cap \widetilde{f}\left(\Gamma_{1}\right)=\emptyset e \Gamma_{1} \prec \widetilde{f}\left(\Gamma_{1}\right)$, ent $\tilde{a} o \tilde{f}(v) \cap \Gamma_{1}=\emptyset$.

Demonstração: Como $\tilde{f}\left(\Gamma_{1}\right) \subset B^{-}$, e por hipóteses temos que $\Gamma_{1} \cap \widetilde{f}\left(\Gamma_{1}\right)=\emptyset$ e $\Gamma_{1} \prec \tilde{f}\left(\Gamma_{1}\right)$, dos Lemas 5.1.6 e 5.1.7, temos que $\widetilde{f}^{-1}\left(\Gamma_{1}\right) \prec \Gamma_{1}$, o que implica por definição que $\left[\widetilde{f}^{-1}\left(\Gamma_{1}\right) \cap V_{a}^{-}\right] \subset$ $\Gamma_{1 a, \text { down }}$ para algum $a<0$.

Por outro lado, notemos que $\Gamma \cup v$ é um conjunto conexo e fechado tal que $(\Gamma \cup v)^{c}$ tem uma componente conexa, denotada $\Omega$ que contém $(-\infty, a) \times\{0\}$.

Agora, por construção, $\Omega \subset p^{-1}\left(\gamma_{E}^{-}\right)$, e por definição de $\Gamma_{1 a, d o w n}$, temos

$$
\bar{\Omega} \supset \overline{\Gamma_{1 a, \text { down }}}
$$

Então, como $\widetilde{f}^{-1}\left(\Gamma_{1}\right) \cap \Gamma_{1}=\emptyset$ e $\left[\widetilde{f}^{-1}\left(\Gamma_{1}\right) \cap V_{a}^{-}\right] \subset \Gamma_{1 a, \text { down }} \subset \bar{\Omega}$ temos só duas possibilidades:

- $\tilde{f}^{-1}\left(\Gamma_{1}\right) \cap v=\emptyset$, o que prova a proposição.

- $\widetilde{f}^{-1}\left(\Gamma_{1}\right) \cap v \neq \emptyset$. Então consideramos um elemento $\Theta \in\left[\widetilde{f}^{-1}\left(\Gamma_{1}\right) \cap \bar{\Omega}\right]=\{$ componentes conexas ilimitadas de $\left.\tilde{f}^{-1} \cap \bar{\Omega}\right\}$. Agora dado que $\tilde{f}^{-1}\left(\Gamma_{1}\right)$ é conexo e ilimitado, e $\tilde{f}^{-1}\left(\Gamma_{1}\right) \cap \Gamma_{1}=\emptyset$, afirmamos que $\Theta \cap v \neq \emptyset$, pois caso contrário, como $\Theta$ é conexo e ilimitado, e não intercepta $\Gamma_{1}$ nem $v$, sendo que $\tilde{f}^{-1}\left(\Gamma_{1}\right)$ sim, teríamos que $\widetilde{f}^{-1}\left(\Gamma_{1}\right)$ tem, ao menos duas componentes conexas ilimitadas, uma interceptando $v$, e a outra que não, e isso contradiz a conexidade de $\widetilde{f}^{-1}\left(\Gamma_{1}\right)$. Logo $\Theta \cap v \neq \emptyset$. Como $\Theta \subset V^{-}$e $\widetilde{f}(\Theta) \subset \Gamma_{1} \subset B^{-}$, obtemos, por ser $\Theta$ ilimitada a esquerda e conexa, que $\Theta \subset B^{-}$, e isso contradiz o fato de $B^{-} \cap v=\emptyset$.

Logo não acontece que $\tilde{f}^{-1}\left(\Gamma_{1}\right) \cap v \neq \emptyset$.

Mais ainda, se $\Gamma_{1} \prec \Gamma_{1}^{+}$, então $\left[\tilde{f} \cap V_{a}^{-}\right] \subset\left[\Gamma_{1}^{+} \cap V_{a}^{-}\right] \subset \Gamma_{1 a, u p}$, implicando que, pela prova do Lema 5.1.3, que $\Gamma_{1} \prec \widetilde{f}\left(\Gamma_{1}\right)$. A última proposição provada vai permitir provar, precisamente o que estamos buscando, pois daí vamos obter o argumento para a contradição que prova o seguinte:

Lema 5.4.1 : $\widetilde{f}\left(\Gamma_{1}\right)$ não está acima de $\Gamma_{1}$, ou seja, acontece uma e só uma das seguintes possibilidades:

- $\tilde{f}\left(\Gamma_{1}\right) \subset \Gamma_{1}$

- $\tilde{f}\left(\Gamma_{1}\right) \prec \Gamma_{1}$

O que implica $\Gamma_{1}=\Gamma_{1}^{+}$ou $\Gamma_{1}^{+} \prec \Gamma_{1}$.

Demonstração: Claramente, se $\Gamma_{1}^{+} \subset \Gamma_{1}$, temos $\Gamma_{1}^{+}=\Gamma_{1}$. Suponha que $\Gamma_{1} \prec \widetilde{f}\left(\Gamma_{1}\right)$. Tomemos $\Omega$ como na demonstração anterior, ou seja, a componente conexa de $\left(\Gamma_{1} \cup v\right)^{c}$, ilimitada a esquerda e que mora no conjunto limitado a direita $(-\infty, 0) \times[0,1] \cap p^{-1}\left(\gamma_{E}^{-}\right)$. Por contrução, $\partial \Omega \subset \Gamma_{1} \cup v$ como $\widetilde{f}\left(\Gamma_{1}\right) \cap \Gamma_{1}=\emptyset$, e por definição de $v$ obtemos $v \cap \widetilde{f}(v)=\emptyset$, tendo alem de tudo isso (pela proposição anterior) que $\widetilde{f}\left(\Gamma_{1}\right) \cap v=\Gamma_{1} \cap \widetilde{f}(v)=\emptyset$, afirmamos que

$$
\tilde{f}(\partial \Omega) \cap \partial \Omega=\emptyset
$$

Pois é claro que $\partial \tilde{f}(\Omega) \subset \widetilde{f}(\partial \Omega)$ e $\widetilde{f}(\partial \Omega) \subset \widetilde{f}\left(\Gamma_{1}\right) \cup \widetilde{f}(v)$ (já que $\widetilde{f}$ é um homeomorfismo). Como $(-\infty, a) \times\{0\} \subset \widetilde{f}((-\infty, a) \times\{0\})$, temos que $\widetilde{f}(v) \cap \bar{\Omega}^{c} \neq \emptyset$.

Resumindo o anterior temos:

- $\tilde{f}\left(\Gamma_{1}\right) \cap \Gamma_{1}=\emptyset$

- $\widetilde{f}(v) \cap \Gamma_{1}=\widetilde{f}\left(\Gamma_{1}\right) \cap v=\emptyset$

- $\widetilde{f}(v) \cap \bar{\Omega}^{c} \neq \emptyset$

- $\Gamma_{1} \prec \Gamma_{1}^{+}$ 
- $(-\infty, a) \times\{0\} \subset \widetilde{f}((-\infty, a) \times\{0\})$

E de tudo isso, concluimos que $\left(\widetilde{f}\left(\Gamma_{1}\right) \cup \widetilde{f}(v)\right) \cap \bar{\Omega}=\emptyset$ e, portanto $\Omega \subset \widetilde{f}(\Omega)$ o qual é uma contradição, provando assim o lema.

Agora temos que $\Gamma_{1}=\Gamma_{1}^{+}$ou $\Gamma_{1}^{+} \prec \Gamma_{1}$. Vamos analisar por separado o caso em que $\Gamma_{1}$ é uma componente injetora e o caso em que $\Gamma_{1}$ é uma componente não injetora, e obter em ambos casos uma contradição. Essa contradição vai a provar que $p\left(B^{-}\right)$é denso em $A$.

\subsection{1 $\Gamma_{1}$ é uma componente injetora}

Lembremos que $\Gamma \subset D$ é uma componente inferior, se $\Gamma \prec \Gamma-(1,0)$, e $\Gamma$ é uma componente superior se $\Gamma-(1,0) \prec \Gamma$.

Como $\Gamma_{1} \subset B^{-}$satisfaz que é injetora, então $\Gamma_{1}-(1,0) \cap \Gamma_{1}=\emptyset$. Como ambos conjuntos são conexos, ilimitados a esquerda, contidos em $V^{-}$, com o complementar conexo, temos que $\Gamma_{1} \prec$ $\Gamma_{1}-(1,0)$ ou $\Gamma_{1}-(1,0) \prec \Gamma$, ou seja $\Gamma_{1}$ é uma componente inferior ou $\Gamma_{1}$ é uma componente superior.

Como $\overline{p\left(\Gamma_{1}\right)} \subset \gamma_{E}^{-}$, temos, pelo falado antes, que $\overline{p\left(\Gamma_{1}\right)} \supset \mathbf{S}^{1} \times\{0\}$, então temos que $d\left(\Gamma_{1}, \mathbf{R} \times\right.$ $\{0\})=0$, o que implica, pelo Lema 5.3.1, que $\Gamma_{1}$ não é uma componente superior, portanto, temos que ela é uma componente inferior.

O Lema 5.4.1, implica que só temos duas possibilidades:

1. $\widetilde{f}\left(\Gamma_{1}\right) \subset \Gamma_{1}$, ou seja $\Gamma_{1}^{+}=\Gamma_{1}$.

2. $\Gamma_{1}^{+} \prec \Gamma_{1}$.

Notemos que, se $\Gamma_{1}^{+}=\Gamma_{1}$, obviamente $\Gamma_{1}^{+}$é uma componente injetora e inferior, e para todo inteiro $k>0, \Gamma_{1}^{+}+(k, 0) \prec \Gamma_{1}$. O último é claro, pois só estou movimentando as componentes conexas ilimitadas e aplicando os Lemas 5.1.2 e 5.1.3.

Se $\Gamma_{1}^{+} \prec \Gamma_{1}$ temos o seguinte:

Afirmação 5.4.1 : Para todo $k$ inteiro positivo, $\Gamma_{1}^{+}+(k, 0) \cap \Gamma_{1}=\emptyset e \Gamma_{1}^{+}+(k, 0) \prec \Gamma_{1}$.

Prova: Se para algum $k_{0}>0, \Gamma_{1}^{+}+\left(k_{0}, 0\right) \cap \Gamma_{1} \neq \emptyset$ então $\Gamma_{1}-\left(k_{0}, 0\right) \cap \Gamma_{1}^{+} \neq \emptyset$. Como $\Gamma_{1}^{+}$é uma componente conexa de $B^{-}$e $\Gamma_{1}-\left(k_{0}, 0\right) \subset B^{-}$é fechado e conexo, $\Gamma_{1}-\left(k_{0}, 0\right) \subset \Gamma_{1}^{+}$, mas isso contradiz o fato de $\Gamma_{1}^{+} \prec \Gamma_{1}$, pois pela proposição 5.2.1, se $\Gamma_{1} \prec \Gamma_{1}-(1,0)$, então $\Gamma_{1} \prec \Gamma_{1}-\left(k_{0}, 0\right)$. Logo temos que $\Gamma_{1} \prec \Gamma_{1}-\left(k_{0}, 0\right)$ e $\Gamma_{1}-\left(k_{0}, 0\right) \subset \Gamma_{1}^{+}$, e como $\Gamma_{1}^{+}$é conexo todo o anterior implicaría que $\Gamma_{1} \prec \Gamma_{1}^{+}$o qual é uma contradição. Obtemos, então, que para todo inteiro positivo $k$, $\Gamma_{1}^{+}+(k, 0) \cap \Gamma_{1}=\emptyset$.

Se o restante da afirmação não for correto, então para algum inteiro positivo $k_{1}, \Gamma_{1} \prec \Gamma_{1}^{+}+\left(k_{1}, 0\right)$ dando assim que $\Gamma_{1}-\left(k_{1}, 0\right) \prec \Gamma_{1}^{+} \prec \Gamma_{1}$, e isso contradiz, novamente, o fato de $\Gamma_{1}$ ser uma componente inferior.

Então o que provamos foi o seguinte, que, em qualquer dos casos 1 e 2 de acima, para todo inteiro $k>0, \Gamma_{1}^{+}+(k, 0) \cap \Gamma_{1}=\emptyset \mathrm{e} \Gamma_{1}^{+}+(k, 0) \prec \Gamma_{1}$. Agora levando em conta esta propriedade em comum, vamos provar a contradição que só deixa a possibilidade de $\Gamma_{1}$ ser uma componente não injetora.

Lema 5.4.2 : Existe um segmento vertical $V_{r}=\{r\} \times[0,1]$ e uma seqüência $\underbrace{n_{i} \rightarrow \infty}_{i \rightarrow \infty}$, tal que $\widetilde{f}^{n_{i}}\left(\Gamma_{1}\right) \cap V_{r} \neq \emptyset$ para todo $i$.

O Teorema 5 segue deste Lema pois existe uma seqüência de pontos $x_{i} \in \widetilde{f}^{n_{i}}\left(\Gamma_{1}\right)$, que além disso, pertencem ao compacto $V_{r}$, logo essa seqüência possui uma subseqüência convergente, mas pela 
definição de $\omega\left(B^{-}\right)$, o fato de ele ser fechado e $\widetilde{f}\left(B^{-}\right) \subset B^{-}$, teríamos que o limíte dessa subseqüência moraria em $\omega\left(B^{-}\right)$, contradizendo o fato de ser vazio, provando assim que $\Gamma_{1}$ só pode ser uma componente não injetora.

Demonstração(do Lema 5.4.2): Como $\Gamma_{1}$ é uma componente conexa inferior $\Gamma_{1} \prec \Gamma_{1}-(1,0)$, o fato de $\widetilde{f}$ preservar a ordem, implica que $\widetilde{f}\left(\Gamma_{1}\right) \prec \widetilde{f}\left(\Gamma_{1}\right)-(1,0)$ (temos que observar que $\widetilde{f}\left(\Gamma_{1}\right)$ pode não ser uma componente conexa, mas, ainda assim, vamos abusar da notação e dizer que ela é uma componente inferior).

Também, por nossa afirmação, temos $\widetilde{f}\left(\Gamma_{1}\right) \prec \Gamma_{1}-(1,0)$, então $\widetilde{f}\left(\Gamma_{1}\right)+(k, 0) \prec \Gamma_{1}$ para todo inteiro positivo $k$. Como $\widetilde{f}\left(\Gamma_{1}\right) \subset B^{-}$, temos para qualquer uma das possibilidades 1 e 2 de antes, $\widetilde{f}\left(\Gamma_{1}\right) \subset \Gamma_{1} \cup \Omega$ (onde $\Omega$ está definido como no començo da seção), pois em qualquer caso temos que se satisfazem uma das duas situações:

- $\tilde{f}\left(\Gamma_{1}\right) \subset \Gamma_{1}$

- $\widetilde{f}\left(\Gamma_{1}\right) \prec \Gamma_{1} \Leftrightarrow\left[\widetilde{f}\left(\Gamma_{1}\right) \cap V_{a}^{-}\right] \subset \Gamma_{1 a, \text { down }} \subset \bar{\Omega}$ onde $a$ e $\Omega$ são definidos, outra vez, como no começo da seção. Lembremos que $\Omega$ é conexo, e $\partial \Omega \subset \Gamma_{1} \cup v$ não intercepta $\widetilde{f}\left(\Gamma_{1}\right)$, como $\widetilde{f}\left(\Gamma_{1}\right)$ é conexo, temos, como antes, que $\widetilde{f}\left(\Gamma_{1}\right) \subset \Omega$.

Bem, agora $\widetilde{f}\left(\Gamma_{1}\right) \cap \Gamma_{1}=\emptyset, \widetilde{f}\left(\Gamma_{1}\right)+(k, 0) \cap \Gamma_{1}=\emptyset$ e $\widetilde{f}\left(\Gamma_{1}\right)+(k, 0) \prec \Gamma_{1}$. Com o anterior provamos também, que se temos $a$ e $\Omega$ como no começo da seção, então

$$
\left[\widetilde{f}\left(\Gamma_{1}\right)+(k, 0) \cap V_{a}^{-}\right] \subset \overline{\Gamma_{1 a, \text { down }}} \subset \bar{\Omega}
$$

Como $\partial \Omega \subset \Gamma_{1} \cup v$ e $\bar{\Omega} \subset(-\infty, 0) \times[0,1]$, tomando $k^{\prime}>0$ suficientemente grande tal que $\widetilde{f}\left(\Gamma_{1}\right)+$ $\left(k^{\prime}, 0\right) \cap\{1\} \times[0,1] \neq \emptyset$, por ser $\tilde{f}\left(\Gamma_{1}\right)+\left(k^{\prime}, 0\right)$ conexo, temos que, por todo o falado acima, $\widetilde{f}\left(\Gamma_{1}\right)+\left(k^{\prime}, 0\right)$ intercepta $\partial \Omega$ no único lugar possível, $v$. Fixemos $k^{\prime}$ tal que aconteça isso.

Denotemos por $\Gamma^{*}$ a uma componente conexa ilimitada de $\widetilde{f}\left(\Gamma_{1}\right)+\left(k^{\prime}, 0\right) \cap \Omega$. Por ser $\widetilde{f}\left(\Gamma_{1}\right)+$ $\left(k^{\prime}, 0\right)$ conexo, e pela escolha de $k^{\prime}$, temos que $\Gamma^{*}$ não está contida em $B^{-}$, pois ele tem interseção não vazia com $v$.

Então, existe um inteiro positivo $a_{1}>0$, tal que $\widetilde{f}^{a_{1}}\left(\Gamma^{*}\right)$ intercepta $(0,+\infty) \times[0,1]$. Para poder continuar, vamos provar agora a seguinte resultado:

Proposição 5.4.3 : Se $\Gamma$ é uma componente conexa de $B^{-}$que satisfaz $\widetilde{f}(\Gamma) \cap \Gamma=\emptyset$ e $\widetilde{f}(\Gamma) \prec \Gamma$ então $\widetilde{f}^{n}(\Gamma) \cap \Gamma=\emptyset$ e $\widetilde{f}^{n}(\Gamma) \prec \Gamma$ para todo inteiro $n>0$.

Demonstração: Por contradição. Suponha que existe algum $n_{0}>1$ (sendo ele o primer inteiro para o qual acontece), tal que $\widetilde{f}^{n_{0}}(\Gamma) \cap \Gamma \neq \emptyset$. Isso significa que $\Gamma, \widetilde{f}(\Gamma), \widetilde{f}^{2}(\Gamma), \ldots, \widetilde{f}^{n_{0}-1}(\Gamma)$ são conjuntos disjuntos (pela escolha de $n_{0}$ ), fechados, ilimitados a esquerda e conexos de $\widetilde{A}$, cada um dos quais possui complementar conexo. Notemos também que, pelo fato de $\Gamma$ ser uma componente conexa e $\tilde{f}^{n_{0}}(\Gamma) \cap \Gamma \neq \emptyset, \tilde{f}^{n_{0}}(\Gamma) \subset \Gamma$.

Agora, o fato de $\prec$ ser um ordem e $\tilde{f}$ preservar ele, obtemos que

$$
\widetilde{f}^{n_{0}-1} \prec \ldots \prec \widetilde{f}^{2}(\Gamma) \prec \widetilde{f}(\Gamma) \prec \Gamma
$$

Por outro lado, como $\Gamma \cap \widetilde{f}^{n_{0}-1}(\Gamma)=\emptyset$, temos $\widetilde{f}^{n_{0}}(\Gamma) \cap \widetilde{f}^{n_{0}-1}(\Gamma)=\emptyset$, e o fato de $\tilde{f}$ preservar ordem implica que

$$
\tilde{f}^{n_{0}}(\Gamma) \prec \widetilde{f}^{n_{0}-1}(\Gamma)
$$

Logo $\widetilde{f}^{n_{0}}(\Gamma) \cap\left(\widetilde{f}^{n_{0}-1}(\Gamma)\right)_{\text {down }}$ possui uma componente conexa ilimitada a esquerda.

Dado que $\widetilde{f}^{n_{0}}(\Gamma) \subset \Gamma$, usando o Lema 5.1.2, obtemos por conexidade que

$$
\Gamma \prec \tilde{f}^{n_{0}-1}(\Gamma)
$$

Mas isso é uma contradição ao falado acima, que provindo de supor que para algum $n_{0}>0$, $\widetilde{f}^{n_{0}}(\Gamma) \cap \Gamma \neq \emptyset$. Logo $\widetilde{f}^{n}(\Gamma) \cap \Gamma=\emptyset$ para todo inteiro positivo $n$ e o resto da proposição sai do fato de $\widetilde{f}$ preservar a ordem.

De todo o trabalhado acima, temos duas posiblidades: 
- Se $\tilde{f}\left(\Gamma_{1}\right) \subset \Gamma_{1}$. Temos que $\tilde{f}^{a_{1}+1}\left(\Gamma_{1}\right) \subset \tilde{f}^{a_{1}}\left(\Gamma_{1}\right) \subset \widetilde{f}\left(\Gamma_{1}\right) \subset \Gamma_{1}$. Como $\tilde{f}\left(\Gamma_{1}\right)+\left(k^{\prime}, 0\right) \cap \Gamma_{1}=\emptyset$, então temos

$$
\tilde{f}^{a_{1}+1}\left(\Gamma_{1}\right)+\left(k^{\prime}, 0\right) \cap \Gamma_{1} \subset \widetilde{f}^{a_{1}}\left(\Gamma_{1}\right)+\left(k^{\prime}, 0\right) \cap \Gamma_{1} \subset \tilde{f}\left(\Gamma_{1}\right)+\left(k^{\prime}, 0\right) \cap \Gamma_{1}=\emptyset
$$

Tomando $\Gamma^{*} \subset \widetilde{f}\left(\Gamma_{1}\right) \pm\left(k^{\prime}, 0\right) \cap \bar{\Omega}$ como acima, claramente temos $\widetilde{f}^{a_{1}}\left(\Gamma^{*}\right) \cap \Gamma_{1}=\emptyset$, e por $\tilde{f}$ preservar $\prec$, obtemos $\tilde{f}^{a_{1}}\left(\Gamma^{*}\right) \prec \Gamma_{1}$.

- Se $\tilde{f}\left(\Gamma_{1}\right) \prec \Gamma_{1}\left(\tilde{f}\left(\Gamma_{1}\right) \cap \Gamma_{1}=\emptyset\right)$, então da proposição 5.4 .3 temos que $\tilde{f}^{a_{1}}\left(\Gamma_{1}\right) \cap \Gamma_{1}=\emptyset$ e $\widetilde{f}^{a_{1}}\left(\Gamma_{1}\right) \prec \Gamma_{1}$.

Como $\Gamma_{1}$ é uma componente inferior e $\tilde{f}$ preserva orientação temos

$$
\widetilde{f}^{a_{1}}\left(\widetilde{f}\left(\Gamma_{1}\right)+\left(k^{\prime}, 0\right)\right)=\widetilde{f}^{a_{1}+1}\left(\Gamma_{1}\right)+\left(k^{\prime}, 0\right) \prec \widetilde{f}^{a_{1}}\left(\Gamma_{1}\right) \prec \Gamma_{1}
$$

E também, $\widetilde{f}^{a_{1}+1}\left(\Gamma_{1}\right)+\left(k^{\prime}, 0\right) \cap \Gamma_{1}=\emptyset$, pois se isso não acontece, teríamos que $\widetilde{f}^{a_{1}+1}\left(\Gamma_{1}\right) \cap \Gamma_{1}-$ $\left(k^{\prime}, 0\right) \neq \emptyset$, então existiría uma componente conexa $\Psi$ (notemos que pelo fato de $\Gamma_{1}$ ser uma componente injetora $\left.\Psi \neq \Gamma_{1}\right)$ contendo $\widetilde{f}^{a_{1}+1}\left(\Gamma_{1}\right)$ e $\Gamma_{1}-\left(k^{\prime}, 0\right)$. Do fato de $\widetilde{f}^{a_{1}+1}\left(\Gamma_{1}\right) \prec \Gamma_{1} \mathrm{e}$ $\Gamma_{1} \prec \Gamma_{1}-\left(k^{\prime}, 0\right)$, obtemos que $\Psi \cap \Gamma_{1, \text { down }}$ e $\Psi \cap \Gamma_{1, \text { up }}$ (pelo Lema2) tem componentes conexas ilimitadas e isso é uma contradição ao lema 5.1.3. Logo Tomando $\Gamma^{*} \subset \widetilde{f}\left(\Gamma_{1}\right)+\left(k^{\prime}, 0\right) \cap \bar{\Omega}$ como antes, estamos nas mesmas condições do ítem anterior.

Resumindo todo o feito, temos que, em qualquer das duas possibilidades, $\widetilde{f}\left(\Gamma_{1}\right) \subset \Gamma_{1}$ ou $\widetilde{f}\left(\Gamma_{1}\right) \prec \Gamma_{1}$, podemos escolher $\Gamma^{*} \subset \widetilde{f}\left(\Gamma_{1}\right)+\left(k^{\prime}, 0\right) \cap \bar{\Omega}$ (onde $\Gamma^{*}$ é uma componente conexa ilimitada e $k^{\prime}>0$ fixado no começo), satisfazendo $\Gamma^{*} \cap(0,+\infty) \times[0,1] \neq \emptyset$ e tal que

$$
\widetilde{f}^{a_{1}}\left(\Gamma^{*}\right) \cap \Gamma_{1}=\emptyset \text { e } \widetilde{f}^{a_{1}}\left(\Gamma^{*}\right) \prec \Gamma_{1}
$$

Então como antes, temos que pelo fato de $\widetilde{f}^{a_{1}}\left(\Gamma^{*}\right) \cap(0,+\infty) \times[0,1] \neq \emptyset$, implica que $\widetilde{f}^{a_{1}}\left(\Gamma^{*}\right) \cap \Omega$ possui uma componente conexa ilimitada $\Gamma^{* *}$ que intercepta $v$ e portanto não está contida em $B^{-}$, logo existe $a_{2}>0$ tal que $\widetilde{f}^{a_{2}}\left(\Gamma^{*}\right) \subset \widetilde{f}^{a_{2}+a_{1}+1}+\left(k^{\prime}, 0\right)$ intercepta $(0,+\infty) \times[0,1]$. Com o mesmo raciocínio obtemos $\Gamma^{* * *} \subset \widetilde{f}^{a_{2}}\left(\Gamma^{* *}\right) \cap \bar{\Omega}$ que intercepta $v$. Novamente, $\Gamma^{* * *}$ não está contida em $B^{-}$ e existe um inteiro $a_{3}>0$ tal que $\widetilde{f}^{a_{3}}\left(\Gamma^{* * *}\right)$ intercepta $(0,+\infty) \times[0,1]$.

Em geral, definimos $n_{i}=a_{1}+a_{2}+\ldots+a_{i}+1$ e temos que $n_{i} \rightarrow \infty$ se $i \rightarrow \infty$, e para todo $i \geq 1$

$$
\widetilde{f}^{n_{i}-1}\left(\widetilde{f}\left(\Gamma_{1}\right)+\left(k^{\prime}, 0\right)\right) \supset \widetilde{f}^{n_{i}-1}\left(\Gamma^{*}\right) \supset \widetilde{f}^{a_{2}+\ldots+a_{i}}\left(\widetilde{f}^{a_{i}}\left(\Gamma^{*}\right)\right) \supset \widetilde{f}^{a_{2}+\ldots+a_{i}}\left(\Gamma^{* *}\right) \supset \ldots \supset \widetilde{f}^{a_{i}}\left(\Gamma^{\text {*...** }}\right)
$$

$\mathrm{E} \tilde{f}^{a_{i}}\left(\Gamma^{i \ldots * \text { *...eces }}\right) \cap(0,+\infty) \times[0,1] \neq \emptyset$. Logo temos que, por construção

$$
\widetilde{f}^{n_{i}}\left(\Gamma_{1}\right) \cap V_{0}-\left(k^{\prime}, 0\right)=\widetilde{f}^{n_{i}}\left(\Gamma_{1}\right) \cap V_{k^{\prime}} \neq \emptyset
$$

E temos assim provado o Lema.

Mas pelo falado antes, isso implicaría que $\omega\left(B^{-}\right) \neq \emptyset$, pois $\widetilde{f}^{n_{i}}\left(\Gamma_{1}\right) \subset B^{-}$para todo $n_{i}$, $\tilde{f}^{n_{i}}\left(\Gamma_{1}\right) \subset \widetilde{f}^{n_{i}}\left(B^{-}\right)$, e $\widetilde{f}^{n+1}\left(B^{-}\right) \subset \widetilde{f}^{n}\left(B^{-}\right)$, logo de todo o anterior temos que $\widetilde{f}^{n}\left(B^{-}\right) \cap V_{k} \neq \emptyset$ para todo $n$, o que implica pelo fato de $V$ ser compacto que $\omega\left(B^{-}\right) \neq \emptyset$, contradizendo o Teorema 2. Logo concluimos que $\Gamma_{1}$ só pode ser uma componente não injetora.

\subsection{2 $\Gamma_{1}$ é uma componente não injetora.}

Como estamos supondo $\overline{p\left(\Gamma_{1}\right)} \subset \gamma_{E}^{-}$, obtemos novamente que $\overline{p\left(\Gamma_{1}\right)} \supset \mathbf{S}^{1} \times\{0\}$.

Afirmação 5.4.2 : Se $\Gamma$ é uma componente não injetora de $B^{-}$e $\widetilde{f}(\Gamma) \subset \Gamma$, então existe um inteiro $k>0$ tal que $\widetilde{f}^{-1}(\Gamma) \subset \Gamma+(k, 0)$. 
Prova: Dado que $\widetilde{f}(\Gamma) \subset \Gamma$, temos $\Gamma \subset \widetilde{f}^{-1}(\Gamma)$. Como $\Gamma \subset V^{-}, \widetilde{f}^{-1}(\Gamma)$ é limitado a sua direita, logo existe $k>0$ tal que $\tilde{f}^{-1}(\Gamma)-(k, 0) \subset V^{-}$.

Se $i \geq 1$ temos

$$
\widetilde{f}^{i}\left(\widetilde{f}^{-1}(\Gamma)-(k, 0)\right)=\widetilde{f}^{i-1}(\Gamma)-(k, 0) \subset \Gamma-(k, 0) \subset \Gamma
$$

Como $\widetilde{f}^{-1}\left(\Gamma-(k, 0)\right.$ é fechado, conexo, ilimitado a esquerda, contido em $V^{-}$e possui todas suas iteradas positivas em $\Gamma$ temos, por definição, que $\widetilde{f}^{-1}(\Gamma)-(k, 0) \subset B^{-}$. Como $\Gamma \subset \widetilde{f}^{-1}(\Gamma)$, temos que $\Gamma-(k, 0) \subset \widetilde{f}^{-1}(\Gamma)+(k, 0)$ e como $\Gamma$ é uma componente conexa não injetora, temos $\widetilde{f}^{-1}(\Gamma)-$ $(k, 0) \cap \Gamma \neq \emptyset$, dado que ambos conjuntos são conexos e $\Gamma$ é uma componente conexa, temos

$$
\tilde{f}^{-1}(\Gamma)-(k, 0) \subset \Gamma \text { ou, o que o mesmo } \tilde{f}^{-1}(\Gamma) \subset \Gamma+(k, 0)
$$

O que prova nossa afirmação.

Agora, $\widetilde{f}^{-1}(\Gamma) \subset \Gamma+(k, 0)$, então $\widetilde{f}^{-2}(\Gamma) \subset \widetilde{f}^{-1}(\Gamma)+(k, 0) \subset \Gamma+(2 k, 0)$ e, em geral, por o mesmo argumento e um proceso inductivo, obtemos que

$$
\tilde{f}^{-n}(\Gamma) \subset \Gamma+(n . k, 0)
$$

Seja agora $\Omega$ definido como na demonstração da proposição 5.4.2, definimos o seguinte conjunto

$$
\Omega_{1}=\bigcup_{n=0}^{\infty} \tilde{f}^{-n}(\Omega)
$$

Logo podemos assegurar que

Lema 5.4.3 : Não acontece que $\widetilde{f}\left(\Gamma_{1}\right) \subset \Gamma_{1}$.

Demonstração: Suponha que isso acontece, então, tomando $\Omega_{1}$ como acima, é claro, pelo fato de $\widetilde{f}$ ser homeomorfismo, que

$$
\tilde{f}^{-1}\left(\Omega_{1}\right) \subset \Omega_{1}
$$

Logo, temos a seguinte:

Proposição 5.4.4: $\Omega_{1} \subset p^{-1}\left(\gamma_{E}^{-}\right)$.

Demonstração: Primeiro, por nossa construção, $\partial \Omega \subset \Gamma_{1} \cup v$, e como $\widetilde{f}^{-1}$ é um homeomorfismo, temos que

$$
\Omega \subset \widetilde{A}-\left(\Gamma_{1} \cup v\right) \Rightarrow \widetilde{f}^{-1}(\Omega) \subset \widetilde{f}^{-1}\left(\widetilde{A}-\left(\Gamma_{1} \cup v\right)\right)=\widetilde{A}-\left(\widetilde{f}^{-1}\left(\Gamma_{1}\right) \cup \widetilde{f}^{-1}(v)\right)
$$

E além disso, como $\widetilde{f}^{-1}(\Omega)$ é aberto, concluimos que sua fronteira está contida em $\left(\widetilde{f}^{-1}\left(\Gamma_{1}\right) \cup \widetilde{f}^{-1}(v)\right)$. Olhemos que, em geral, o anterior pode ser feito para $\partial\left(\tilde{f}^{-i}(\Omega)\right)$, sendo $i$ qualquer inteiro positivo, logo podemos escrever (pois é mas conveniente para a nossa prova) que

$$
\partial\left(\tilde{f}^{-i}(\Omega)\right) \subset \bigcup_{n=0}^{\infty} \tilde{f}^{-n}\left(\Gamma_{1} \cup v\right)=\left(\bigcup_{n=0}^{\infty} \tilde{f}^{-n}\left(\Gamma_{1}\right)\right) \cup\left(\bigcup_{n=0}^{\infty} \tilde{f}^{-n}(v)\right)
$$

Agora, como $\tilde{f}^{-i}(\Omega) \cap \Omega \neq \emptyset$, só temos que provar que $\left(\bigcup_{n=0}^{\infty} \widetilde{f}^{-n}\left(\Gamma_{1}\right)\right) \cup\left(\bigcup_{n=0}^{\infty} \tilde{f}^{-n}(v)\right) \subset$ $p^{-1}\left(\gamma_{E}^{-1}\right)$, logo a equação anterior daria que $\tilde{f}^{-i}(\Omega) \subset p^{-1}\left(\gamma_{E}^{-}\right)$para todo inteiro positivo $i$, dando como resultado $\Gamma_{1} \subset \gamma_{E}^{-}$.

Então o próximo passo é analisar os conjuntos $\left(\bigcup_{n=0}^{\infty} \tilde{f}^{-n}\left(\Gamma_{1}\right)\right)$ e $\left(\bigcup_{n=0}^{\infty} \widetilde{f}^{-n}(v)\right)$. 
- $\bigcup_{n=0}^{\infty} \tilde{f}^{-n}\left(\Gamma_{1}\right)$.

Pelo fato de $\Gamma_{1}$ ser não injetora e como estamos supondo $\widetilde{f}\left(\Gamma_{1}\right) \subset \Gamma_{1}$, temos da afirmação 5.4 .2 , que para todo $n>0$, existe $k>0$ inteiro (fixo) tal que

$$
\tilde{f}^{-n}\left(\Gamma_{1}\right) \subset \Gamma_{1}+(n . k, 0)
$$

E também, estamos supondo que $\overline{p\left(\Gamma_{1}\right)} \supset \mathbf{S}^{1} \times\{0\}$, logo obtemos daí que para todo inteiro positivo $n$ se cumpre

$$
\Gamma_{1}+(n, 0) \subset p^{-1}\left(\gamma_{E}^{-}\right)
$$

então, juntando o anterior temos que

$$
\bigcup_{n=0}^{\infty} \tilde{f}^{-n}\left(\Gamma_{1}\right) \subset \bigcup_{n=0}^{\infty}\left(\tilde{f}^{-n}\left(\Gamma_{1}\right)+(n . k, 0)\right) \subset p^{-1}\left(\gamma_{E}^{-}\right)
$$

que é o procurado.

- $\bigcup_{n=0}^{\infty} \tilde{f}^{-n}(v)$

Da escolha de $v$ temos, pela mesma justificativa que no Teorema 2 , que $\widetilde{f}^{-1}(v) \cap v=\emptyset$, e como $v \cap B^{-}=\emptyset$, obtemos $\widetilde{f}^{-1}(v) \cap \Gamma_{1}=\emptyset$. Também temos, por construção e as propriedades de $\widetilde{f}$ respectivamente, as seguintes inclusões

$$
\Omega \supset(-\infty, a) \times\{0\} \quad(-\infty, a) \times\{0\} \supset \widetilde{f}^{-1}((-\infty, a) \times\{0\})
$$

Implicando que $\tilde{f}^{-1}(v) \cap \Omega \neq \emptyset$, logo pelo anterior $\tilde{f}^{-1}(v) \subset \Omega \subset p^{-1}\left(\gamma_{E}^{-}\right)$.

Então, $\widetilde{f}^{-2}(v) \subset \widetilde{f}^{-1}(\Omega)$, e a fronteira deste último conjunto está contida em $\widetilde{f}^{-1}\left(\Gamma_{1}\right) \cup$ $\widetilde{f}^{-1}(v) \subset p^{-1}\left(\gamma_{E}^{-}\right.$(pelo anterior). Como $\widetilde{f}^{-1}(\Omega)$ é conexo e tem interseção não vazia com $\Omega$ obtemos que

$$
\tilde{f}^{-2}(v) \subset \tilde{f}^{-1}(\Omega) \subset p^{-1}\left(\gamma_{E}^{-}\right)
$$

E por um argumento inductivo, obtemos que

$$
\widetilde{f}^{-n}(v) \subset \widetilde{f}^{-n+1}(\Omega) \subset p^{-1}\left(\gamma_{E}^{-}\right)
$$

Para todo inteiro $n$ maior do que 1. Isso acaba provando a proposição.

Agora como $\tilde{f}^{-1}\left(\Omega_{1}\right) \subset \Omega_{1}, \Omega_{1}$ é denso, mas $\Omega_{1} \subset p^{-1}\left(\gamma_{E}^{-}\right)$, e isso é uma contradição.

Como resultado do anterior, só é posível que, sendo $\Gamma_{1}$ uma componente não injetora, $\widetilde{f}\left(\Gamma_{1}\right) \prec$ $\Gamma_{1}$. Mas se isso acontece, temos a versão da proposição 5.4 .2 para uma componente não injetora:

Lema 5.4.4 : Se $\Gamma_{1}$ é uma componente não injetora de $B^{-}$, existe um segmento vertical $V_{r}=$ $\{r\} \times[0,1]$ e uma seqüência $n_{i} \rightarrow \infty$, quando $i \rightarrow \infty$ tal que $\widetilde{f}^{n_{i}}\left(\Gamma_{1}\right) \cap V_{r} \neq \emptyset$ para todo inteiro $i \geq 1$.

Demonstração: Como $\tilde{f}\left(\Gamma_{1}\right) \prec \Gamma_{1}$, temos, como na Proposição ??, a e $\Omega$ tais que

$$
\left[\tilde{f}\left(\Gamma_{1}\right) \cap V_{a}^{-}\right] \subset \overline{\Gamma_{1 a, \text { down }}} \subset \bar{\Omega}
$$

e, pelo mesmo argumento que no Lema 5.4.2, obtemos $\widetilde{f}\left(\Gamma_{1}\right) \subset \Omega$.

Agora,se para alguns inteiros $n_{0}$ e $k_{0}$, temos que $\widetilde{f}^{n_{0}}\left(\Gamma_{1}\right)+\left(k_{0}, 0\right) \cap \Gamma_{1} \neq \emptyset$, então $\widetilde{f}^{n_{0}}\left(\Gamma_{1}\right) \cap$ $\Gamma_{1}-\left(k_{0}, 0\right) \neq \emptyset$, e como $\Gamma_{1}$ é não injetora, o anterior implica que $\tilde{f}^{n_{0}}\left(\Gamma_{1}\right) \cap \Gamma_{1} \neq \emptyset$. Dado também que $\Gamma_{1}$ é uma componente conexa, obtemos $\widetilde{f}^{n_{0}}\left(\Gamma_{1}\right) \subset \Gamma_{1}$ o qual provamos, no Lema 5.4.1, que não acontece.

Logo para todos os inteiros positivos $k$ e $n$ temos 


$$
\widetilde{f}^{n}\left(\Gamma_{1}\right)+(k, 0) \cap \Gamma_{1}=\emptyset
$$

Além disso, pelo fato de $\tilde{f}$ preservar orientação e $\Gamma_{1} \subset \Gamma_{1}+(k, 0)$ temos $\tilde{f}\left(\Gamma_{1}\right) \subset \widetilde{f}\left(\Gamma_{1}\right)+(k, 0)$. Também temos que $\tilde{f}$ preserva $\prec$, e $f\left(\Gamma_{1}\right) \prec \Gamma_{1}$, então, do Lema 5.1.7 podemos assegurar que

$$
\tilde{f}^{n}\left(\Gamma_{1}\right) \prec \ldots \prec \widetilde{f}\left(\Gamma_{1}\right) \prec \Gamma_{1}
$$

Logo, como $\tilde{f}^{n}\left(\Gamma_{1}\right)+(k, 0) \cap \Gamma_{1}=\emptyset$, afirmamos que $\widetilde{f}^{n}\left(\Gamma_{1}\right)+(k, 0) \prec \Gamma_{1}$ pois se isso não acontece temos que $\Gamma_{1}-(k, 0) \prec \widetilde{f}^{n}\left(\Gamma_{1}\right)$ e $\widetilde{f}^{n}\left(\Gamma_{1}\right) \prec \Gamma_{1}$ o qual, por ser $\Gamma_{1}$ não injetora, é uma contradição. Então temos que $\widetilde{f}^{n}\left(\Gamma_{1}\right)+(k, 0) \cap \Gamma_{1}=\emptyset$ e $\widetilde{f}^{n}\left(\Gamma_{1}\right)+(k, 0) \prec \Gamma_{1}$. Logo estamos na mesma situação do Lema 5.4.2, e a prova continua da mesma forma.

Como no Lema 5.4.2, concluimos que $\omega\left(B^{-}\right) \neq \emptyset$, o qual contradiz o Teorema 2.

Mas acabamos de obter, supondo $p\left(\Gamma_{1}\right)$ não denso, uma contradição, pois $\Gamma_{1}$ não pode ser injetora, e também não pode ser não injetora. Logo, $p\left(\Gamma_{1}\right)$ tem que ser denso em $A$, provando assim o Teorema 4.

\subsection{Demonstração do Teorema 5}

Lembremos antes de começar, que procuramos provar. Existe uma componente conexa $\Gamma$ de $B^{-}$, tal que $p(\Gamma)$ é densa em $A, \widetilde{f}(\Gamma) \subset \Gamma, \Gamma-(1,0) \subset \Gamma$ e existe um inteiro positivo $k$ tal que $\widetilde{f}^{-1}(\Gamma) \subset \Gamma+(k, 0)$, ou seja $f(p(\Gamma))=p(\Gamma)$.

A idéia para a prova deste resultado é a seguinte: Vamos fazer a mesma construção que no começo do da seção anterior, para achar a menor componente conexa de $B^{-}$(dadas certas condições), logo, vamos ver que condições pode satisfazer essa componente conexa. Depois, vamos fazer o mesmo para (em certas condições), a maior componente conexa de $B^{-}$, e vamos provar que uma das duas componentes, satisfaz o Teorema 5.

Seja $\epsilon>0$ tal que para todo $(\widetilde{x}, \widetilde{y}) \in \mathbf{R} \times\{[0, \epsilon] \cup[1-\epsilon, 1]\}$ vale que

$$
p_{1} \circ \widetilde{f}(\widetilde{x}, y)>\widetilde{x}+\sigma
$$

para algum $\sigma>0$ fixo.

Como já sabemos, $\overline{p\left(B^{-}\right)}=A$, então temos que existe um $b$ suficientemente negativo tal que

$$
\Theta \cap\{b\} \times[0, \epsilon] \neq \emptyset
$$

para alguma componente conexa $\Theta$ de $B^{-}$.

Então vamos considerar a componente conexa mais baixa de $B^{-}$em $\{b\} \times[0, \epsilon]$.

Então, dado que $B^{-}$é fechado, existe um $\delta$ satisfazendo $0<\delta \leq \epsilon$ tal que $(b, \delta) \in B^{-}$e para todo $0 \leq \widetilde{y}<\delta$ temos $(b, \widetilde{y}) \notin B^{-}$. Denotemos por $v$ el segmento $\{b\} \times[0, \delta)$.

Seja agora $\Gamma_{1}$ a componente conexa de $B^{-}$que contém $(b, \delta)$. Lembrando os resultados das proposições 5.4.1 e 5.4.2 e do Lema 5.4.1, se $\Gamma_{1} \prec \widetilde{f}\left(\Gamma_{1}\right)$ então o conjunto $\Omega$ (o qual é uma componente conexa aberta de $\left(\Gamma_{1} \cup v\right)^{c}$ ilimitada a esquerda, que está em $(\underset{\widetilde{f}}{\cup}, 0) \times[0,1]$ e contém $(-\infty, b) \times\{0\})$ satisfaz que: $\Omega \subset \widetilde{f}(\Omega)$ e isso contradiz a transitividade da $\widetilde{f}$.

Logo, só pode acontecer que $\tilde{f}\left(\Gamma_{1}\right) \subset \Gamma_{1}$ ou $\widetilde{f}\left(\Gamma_{1}\right) \prec \Gamma_{1}$. Vamos analisar todas as possibilidades que podem acontecer com a componente conexa $\Gamma_{1}$ :

1. $\Gamma_{1}$ é uma componente injetora inferior

2. $\Gamma_{1}$ é uma componente injetora superior

3. $\Gamma_{1}$ é uma compontente não injetora

No caso 1 , se $\tilde{f}\left(\Gamma_{1}\right) \subset \Gamma_{1}$ ou $\widetilde{f}\left(\Gamma_{1}\right) \prec \Gamma_{1}$, e no caso 3 , se $\widetilde{f}\left(\Gamma_{1}\right) \prec \Gamma_{1}$, os Lemas 5.4.2 e 5.4.4, implicariam, novamente, que $\omega\left(B^{-}\right) \neq \emptyset$, e isso contradiz o Teorema 2.

Então, só pode acontecer que $\Gamma_{1}$ seja uma componente injetora superior, ou $\Gamma_{1}$ seja uma compoenente não injetora e $\widetilde{f}\left(\Gamma_{1}\right) \subset \Gamma_{1}$. Suponha então que $\Gamma_{1}$ seja uma componente injetora superior. Temos as seguintes possibilidades: 
- $d\left(\Gamma_{1}, \mathbf{R} \times\{1\}\right)>0$

- $d\left(\Gamma_{1}, \mathbf{R} \times\{1\}\right)=0$

Então temos o seguinte

Lema 5.5.1 : Se $\Gamma_{1}$ é uma componente injetora superior, então $d\left(\Gamma_{1}, \mathbf{R} \times\{1\}\right)=0$

Demonstração: Como $\Gamma_{1}$ é uma componente injetora superior, o Lema 5.3.1 implica que

$$
d\left(\Gamma_{1}, \mathbf{R} \times\{0\}\right)>0
$$

Agora, suponha que $d\left(\Gamma_{1}, \mathbf{R} \times\{1\}\right)>0$, então existe $\epsilon_{1}$ tal que

$$
\Gamma_{1} \cap \mathbf{R} \times\left\{\left[0, \epsilon_{1}\right] \cup\left[1-\epsilon_{1}, 1\right]\right\}=\emptyset
$$

Dado que $\tilde{f}$ é transitiva, $f$ também o é, logo existe um ponto $z \in \mathbf{S}^{1} \times\left[1-\frac{\epsilon_{1}}{2}, 1\right]$ e um inteiro $n>0$ tal que $f^{-n}(z) \in \mathbf{S}^{1} \times\left[0, \frac{\epsilon_{1}}{2}\right]$. Dos resultados do capitulo anterior, temos que $\widetilde{f}\left(\Gamma_{1}\right) \subset \Gamma_{1}$ ou $\widetilde{f}\left(\Gamma_{1}\right) \prec \Gamma_{1}$, então, pela proposição 5.4 .3 , e os Lemas 5.1 .5 e 5.1.6, obtemos que

$$
\widetilde{f}^{n}\left(\Gamma_{1}\right) \subset \Gamma_{1} \text { ou } \tilde{f}^{n}\left(\Gamma_{1}\right) \prec \Gamma_{1}
$$

Agora, lembrando a definição de $m_{\Gamma}$ (ver o começo do capítulo), tomemos $d \in \mathbf{R}$ tal que $\tilde{f}^{-i}\left(V_{d}\right) \subset$ $V_{m_{\Gamma_{1}}-1}^{-}$, para $i=0,1, \ldots, n$ e $\widetilde{f}^{n}\left(\Gamma_{1}\right) \cap V_{d}^{-} \subset \Gamma_{1} \cup \Gamma_{1, \text { down }}$ (esto último é obtido da Afirmação 5.1 .2 provada no inicio).

Tomemos $\widetilde{z} \in V_{d}^{-}$um ponto tal que $p(\widetilde{z})=z$ e seja $k$ o segmento vertical que tem por extremos a $\widetilde{z}$ e um ponto $\widetilde{z}_{1} \in \mathbf{R} \times\{1\}$ (notemos que $k \subset \Gamma_{1, \text { up }} \cap V_{d}^{-}$).

Como $\widetilde{f}^{-n}(\widetilde{z}) \in \mathbf{R} \times\left[0, \frac{\epsilon_{1}}{2}\right] \cap V_{m_{\Gamma_{1}-1}}^{-}$, claramente temos que $\widetilde{f}^{-n}(\widetilde{z}) \in \Gamma_{1, \text { down }}$. Agora como $\widetilde{f}^{-n}\left(V_{d}\right) \subset V_{m_{\Gamma_{1}-1}}^{-}$obtemos que $\widetilde{f}^{-n}(k) \cap V_{m_{\Gamma_{1}}}=\emptyset$. Pela escolha de $\widetilde{z}_{1}$, temos que $\widetilde{f}^{-n}\left(\widetilde{z}_{1}\right) \notin \Gamma_{1, \text { down }}$, como $\tilde{f}^{-n}(\widetilde{z}) \in \Gamma_{1, \text { down }}$ e $k$ é conexo obtemos

$$
\widetilde{f}^{-n}(k) \cap \partial\left(\Gamma_{1, \text { down }}\right) \neq \emptyset
$$

Mas $\partial\left(\Gamma_{1, \text { down }}\right) \subset \Gamma_{1} \cup V_{m_{\Gamma_{1}}}$, e, como $\tilde{f}^{-n}(k) \cap V_{m_{\Gamma_{1}-1}}=\emptyset$, obtemos que $\tilde{f}^{-n}(k) \cap \Gamma_{1} \neq \emptyset$, o que implica que

$$
k \cap \widetilde{f}^{n}\left(\Gamma_{1}\right) \neq \emptyset
$$

E isso é uma contradição, pois $k \subset \Gamma_{1, u p} \cap V_{d}^{-}$e $\tilde{f}^{n}\left(\Gamma_{1}\right) \cap V_{d}^{-} \subset \Gamma_{1} \cup \Gamma_{1, \text { down. Esta contradição }}$ prova o Lema.

Tomemos o $\epsilon>0$ definido no começo do capítulo, e um $c$ suficientemente negativo tal que

$$
\Gamma_{1} \cap\{c\} \times[1-\epsilon, 1] \neq \emptyset
$$

Como acima, pelo fato de $B^{-}$ser fechado, existe $\mu$ tal que $0<\mu \leq \epsilon,(c, 1-\mu) \in B^{-}$, e para todo $\widetilde{y}$, com $1-\mu \leq \widetilde{y}<1$, temos que $(c, \widetilde{y}) \notin B^{-}$, ou seja, $(c, 1-\mu)$ é o maior ponto de $B^{-}$em $\{c\} \times[1-\epsilon, 1]$. Denotamos agora por $w$ ao segmento $\{c\} \times(1-\mu, 1]$.

Seja agora $\Gamma_{2}$ a componente conexa de $B^{-}$que contém $(c, 1-\mu)$. Notemos que as provas do Lema 5.4.2 e do Lema 5.4.4 forom feitas para uma componente conexa $\Gamma$ satisfazendo que $\overline{p(\Gamma)} \supset \mathbf{S}^{1} \times\{0\}$, mas supondo que $\overline{p(\Gamma)} \supset \mathbf{S}^{1} \times\{1\}$, os resultados dos Lemas 5.4 .2 e 5.4.4 são análogos, só que agora, eles impedem que $\Gamma_{2}$ seja uma componente injetora superior (tem-se que refazer todas as provas desde o começo, só supondo agora que existe uma componente conexa de $B^{-}$que satisfaz $p(\Gamma) \subset \gamma_{E}^{+}$). Agora se $\Gamma_{1}$ é injetora (superior), pelo falado, $\Gamma_{2} \neq \Gamma_{1}$, e portanto $\Gamma_{1} \cap \Gamma_{2}=\emptyset$.

Então, $\Gamma_{2}$, ou é uma componente conexa não injetora, ou é uma componente injetora inferior. Neste último caso, temos, por ser ela injetora, e $\Gamma_{2} \prec \Gamma_{2}-(1,0)$, que $d\left(\Gamma_{2}, \mathbf{R} \times\{1\}\right)>0$ (Lema 5.3.1), logo, como $d\left(\Gamma_{1}, \mathbf{R} \times\{1\}\right)=0$ (pois estamos supondo $\Gamma_{1}$ injetora superior), não é posível 
que $\Gamma_{1} \prec \Gamma_{2}$. Isso implica que $\Gamma_{2} \prec \Gamma_{1}$. Mas o anterior implica que (pelo fato de $(c, 1-\mu) \in \Gamma_{2} \mathrm{e}$ ele ser o maior ponto de $B^{-}$no segmento $\left.\{c\} \times[1-\epsilon, 1]\right)$ temos que $\Gamma_{2}$ intercepta $v$, o qual é uma contradição, pois $v$ não pertence a $B^{-}$. Outra vez, pelo mesmo argumento, temos que $\Gamma_{2}$ é uma componente não injetora e $\widetilde{f}\left(\Gamma_{2}\right) \subset \Gamma_{2}$.

Notemos que falta provar a densidade, pois todo o resto já foi provado. O próximo Lema conlui a prova do Teorema 5 , pois ou $\Gamma_{1}$ é uma componente não injetora e $\widetilde{f}\left(\Gamma_{1}\right) \subset \Gamma_{1}$, ou, caso de $\Gamma_{1}$ ser injetora, temos, pelo falado acima que $\Gamma_{2}$ é não injetora e $\tilde{f}\left(\Gamma_{2}\right) \subset \Gamma_{2}$.

Lema 5.5.2 : Se $\Gamma$ é uma componente não injetora de $B^{-}$tal que $\widetilde{f}(\Gamma) \subset \Gamma$, então $\overline{p(\Gamma)}=A$.

Demonstração: Aqui, notemos que o conjunto $\Gamma$ satisfaz as mesmas propriedades que o conjunto $D$. E portanto valem os mesmos resultados que para $D$, logo $\overline{p(\Gamma)} \supset \mathbf{S}^{1} \times\{0\}$ ou $\overline{p(\Gamma)} \supset \mathbf{S}^{1} \times\{0\}$.

Então suponha que (o outro caso é o mesmo) que

$$
\overline{p(\Gamma)} \supset \mathbf{S}^{1} \times\{0\}
$$

os resultados acerca de $p(D)$ mostram que se $p(\Gamma)$ não é denso em $A$, então existe uma uma curva fechada simples $\gamma \subset A-\left\{\mathbf{S}^{1} \times\{0\} \cup \mathbf{S}^{1} \times\{1\}\right\}$, homotópicamente não trivial e tal que $\overline{p(\Gamma)} \cap \gamma=\emptyset$. Dado que $p^{-1}(\gamma)$ possui exactamente duas componentes conexas, $\gamma^{-}$e $\gamma^{+}$contendo $\mathbf{R} \times\{0\}$ e $\mathbf{R} \times\{1\}$ respectivamente, $p(\Gamma)$ é conexo e $\overline{p(\Gamma)} \supset \mathbf{S}^{1} \times\{0\}$, temos que $\Gamma \subset p^{-1}\left(\gamma^{-}\right)$.

Como $\Gamma$ é fechado e $\mathbf{S}^{1} \times\{0\} \subset \overline{p(\Gamma)}$, podemos achar um ponto $\left(c^{\prime}, \delta^{\prime}\right) \in \Gamma$ tal que, tomando $\epsilon$ como no começo do capítulo satisfaça:

- $\delta^{\prime}<\epsilon$.

- Se $v^{\prime}=\left\{c^{\prime}\right\} \times\left[0, \delta^{\prime}\right)$ então $\Gamma \cap v^{\prime}=\emptyset \mathrm{e} \overline{v^{\prime}} \subset p^{-1}\left(\gamma^{-}\right)$.

Agora, escolhamos $\Omega^{\prime}$ como a componente conexa de $\left(\Gamma \cup v^{\prime}\right)^{c}$ que contém $\left(-\infty, c^{\prime}\right) \times\{0\}$ e consideramos o seguinte conjunto:

$$
\Omega_{\text {sat }}=\bigcup_{n=0}^{\infty} \tilde{f}^{-n}\left(\Omega^{\prime}\right)
$$

Agora pelos mesmos argumento que na Proposição 5.4.4, vamos obter que $\Omega_{\text {sat }} \subset p^{-1}\left(\gamma^{-}\right)$. Das propriedades de $\widetilde{f}$, temos que $\widetilde{f}^{-1}\left(\Omega_{\text {sat }}\right) \subset \Omega_{\text {sat }}$, o que implica que $\Omega_{\text {sat }}$ é denso em $\widetilde{A}$ e está contido num conjunto não denso, o que é uma contradição e prova o lema.

Com este último Lema, provamos o Teorema 5, pois provamos que uma das duas componentes conexas, $\Gamma_{1} \Gamma_{2}$, tem projeção densa em $A, \widetilde{f}\left(\Gamma_{i}\right) \subset \Gamma_{i}(i=1$ ou $i=2)$ e é não injetora, isso implica(pelo já provado), que existe $k$ inteiro positivo tal que $\tilde{f}^{-1}(\Gamma) \subset \Gamma+(k, 0)$ e o resto do resultado.

\subsection{Demonstração do Teorema 6}

Nesta seção, vamos provar que toda componente conexa $\Gamma$ de $B^{-}$é uma componente não injetora, ou seja, vamos provar que $\Gamma-(1,0) \subset \Gamma$.

Vamos necesitar de um resultado acerca das componentes conexas $\Gamma_{1}$ e $\Gamma_{2}$ com as que trabalhamos na seção anterior.

Proposição 5.6.1 : Ambas componentes conexas $\Gamma_{1}$ e $\Gamma_{2}$ são componentes não injetoras.

Demonstração: Se a proposição for falsa para $\Gamma_{1}$, como já provamos, $\Gamma_{1}$ tem que ser uma componente injetora superior. Como $\Gamma_{2}$ não corta $v$ e $\Gamma_{1}$ não corta $w$ (pelas definições de $v$ e $w$ ), temos que ter $\Gamma_{1} \prec \Gamma_{2}$ e, pelo fato de ter $\Gamma_{1} \cap \Gamma_{2}=\emptyset$, temos

$$
d\left(\Gamma_{1}, \mathbf{R} \times\{0\}\right)>0
$$

e isso contradiz o Lema 5.5.2, pois $p\left(\Gamma_{2}\right)$ é densa em $A$. Então $\Gamma_{1}$ é uma componente não injetora. Agora supondo que $\Gamma_{2}$ é não injetora, como já provamos, $\Gamma_{2}$ só pode ser uma componente injetora inferior, com o qual, $\Gamma_{1}$ é não injetora e $\widetilde{f}\left(\Gamma_{1}\right) \subset \Gamma_{1}$, logo, $\overline{p\left(\Gamma_{1}\right)}=A$, mas do fato de ter $\Gamma_{1} \cap \Gamma_{2}=\emptyset$ temos que 


$$
d\left(\Gamma_{2}, \mathbf{R} \times\{1\}\right)>0
$$

o que é, outra vez, uma contradição.

Estamos agora em condições de provar que $\Gamma-(1,0) \subset \Gamma$ para toda componente conexa $\Gamma$ de $B^{-}$. A idéia da prova, é supor que $\Gamma$ não satisfaz o anterior, logo, só pode acontecer que $\Gamma$ seja uma componente injetora, a qual também podemos supor, sem perda de generalidade, como uma componente inferior. Logo, trabalhando com $\Gamma$ e a proposição anterior, vamos provar, que nestas hipóteses, $\omega\left(B^{-}\right) \neq \emptyset$, o qual contradiz o Teorema 2 e prova o resultado.

Então, supondo que $\Gamma$ é uma componente injetora inferior, consideramos o segmento vertical $v=\{c\} \times[0, \epsilon)$ satisfazendo o seguinte:

1. $0<\epsilon \leq \epsilon^{\prime}$, onde $\epsilon^{\prime}$ é tal que $\forall(\widetilde{x}, \widetilde{y}) \in \mathbf{R} \times\left[0, \epsilon^{\prime}\right], p_{1} \circ \widetilde{f}(\widetilde{x}, \widetilde{y})>\widetilde{x}+\sigma$, para algum $\sigma>0$ fixo.

2. $v \subset \Gamma_{\text {down }}$.

3. $v \cap B^{-} \neq \emptyset$

O anterior é posível pois $\overline{p\left(B^{-}\right)}=A$ e se $\{c\} \times[0, b) \subset \Gamma_{\text {down }}$ e $(c, b) \in \Gamma$, então $B^{-} \cap\{c\} \times[0, b) \neq$ $\emptyset$ (pelos resultados anteriores). Então podemos escolher um $c$ suficientemente negativo tal que $\{c\} \times\left[0, \epsilon^{\prime}\right] \cap B^{-} \neq \emptyset$, sendo $\epsilon^{\prime}$ obtido de 1) de acima. Se $\{c\} \times\left[0, \epsilon^{\prime}\right) \subset \Gamma_{\text {down }}$, por 2) e 3), estamos nas condições que precissamos. Se isso não acontece, seja $0<\epsilon<\epsilon^{\prime}$ tal que $v=\{c\} \times[0, \epsilon) \subset \Gamma_{\text {down }}$ $\mathrm{e}(c, \epsilon) \in \Gamma$.

Do feito acima, podemos escolher $\Theta$ como a menor componente conexa de $B^{-}$em $v$ e por $w=\{c\} \times[0, \delta) \subset v$ o segmento vertical tal que $w \cap B^{-}=\emptyset$ e $(c, \delta) \in \Theta$. Do Teorema 5 temos que $\Theta$ satisfaz o seguinte:

- $\Theta$ é não injetora.

- $\tilde{f}(\Theta) \subset \Theta$.

- $\overline{p(\Theta)}=A$

Agora, se $\Theta \prec \Gamma$, já provamos antes que existe um número real $d$ tal que $\Theta \cap V_{d}^{-} \subset \Gamma_{\text {down }}$, como $\Gamma$ é uma componente injetora inferior, isso implica que $d(\Gamma, \mathbf{R} \times\{1\})>0$, mas do anterior, temos que

$$
d(\Theta, \mathbf{R} \times\{1\})>0
$$

Mas isso contradiz o fato de $p(\Theta)$ ser denso em $A$.

Então podemos assumir que $\Gamma \prec \Theta$. Como antes consideramos $\Omega$ a componente conexa de $(\Theta \cup w)^{c}$ que contém $(-\infty, c) \times\{0\}$, dos resultados obtidos nos capítulos anteriores, temos

$$
\bar{\Omega} \supset \overline{\Theta_{c, \text { down }}}
$$

e como $\Gamma \prec \Theta$, temos $\left[\Gamma \cap V_{c}^{-}\right] \subset \Theta_{c, \text { down }}$. Então, dado que $\Gamma$ é conexo, $\Gamma \cap \Omega \neq \emptyset$ e $\Gamma \cap \partial \Omega \subset$ $\Gamma \cap(\Theta \cup w)=\emptyset$, temos

$$
\Gamma \subset \Omega
$$

Vamos dividir o resto da prova em dois passos:

Passo 1: Aqui vamos provar que para qualquer par de inteiros $n>0$ e $k \geq 0, \widetilde{f}^{n}(\Gamma)+(k, 0)$ é disjunto de $\Theta$ e $\widetilde{f}^{n}(\Gamma) \subset \Omega \Rightarrow \widetilde{f}^{n}(\Gamma)+(k, 0) \prec \Theta$.

Como $\Gamma \prec \Theta$, temos que para qualquer inteiro positivo $n$, ou $\widetilde{f}^{n}(\Gamma) \subset \Theta$ ou $\widetilde{f}(\Gamma) \cap \Theta=\emptyset$ e $\widetilde{f}^{n}(\Gamma) \prec \Theta$ (isso pois $\widetilde{f}$ preserva a ordem e $\widetilde{f}(\Theta) \subset \Theta$ ).

Primeiro, suponha que $\widetilde{f}(\Gamma) \subset \Theta$, isto implica que $\widetilde{f}^{-1}(\Theta) \supset \Gamma$, logo, se $\widetilde{f}^{-1}(\Theta) \cap V=\emptyset$, então $\tilde{f}^{-1}(\Theta)$ está contida numa componente conexa de $B^{-}$, por nossa suposição, temos que $\widetilde{f}^{-1}(\Theta)=\Gamma$, o qual é uma contradição, pois $\Gamma$ é injetora e $\Theta$ não.

Então $\widetilde{f}^{-1}(\Theta) \cap V \neq \emptyset$. Seja $\Gamma^{\prime}$ a componente conexa de $\widetilde{f}^{-1}(\Theta) \cap V^{-}$que contém $\Gamma$. Do fato de 
$\widetilde{f}^{-1}(\Theta)$ ser conexo, temos que $\Gamma^{\prime}$ intercepta $V$, contém $\Gamma$ e está contida em $B^{-}$(pois $\Gamma$ é ilimitada a esquerda). Então $\Gamma^{\prime}=\Gamma$ e isso é uma contradição pois $\Gamma \subset \Omega$ e $\Omega \cap V=\emptyset$. Logo, temos que $\widetilde{f}(\Gamma) \cap \Theta=\emptyset$ e por o falado acima, $\widetilde{f}(\Gamma) \prec \Theta$ e então $\widetilde{f}(\Gamma) \subset \Omega$.

Agora, podemos assegurar que $\widetilde{f}(\Gamma)=\Gamma^{+}$(lembremos que $\Gamma^{+}$é a componente conexa de $B^{-}$ que contém $\widetilde{f}(\Gamma))$, pois se isso não fosse certo, então $\widetilde{f}^{-1}\left(\Gamma^{+}\right) \supset \Gamma$ não é uma componente conexa de $B^{-}$, $\operatorname{logo} \widetilde{f}^{-1}\left(\Gamma^{+}\right) \cap V \neq \emptyset$, o que implica que $\widetilde{f}^{-1}\left(\Gamma^{+}\right) \cap w \neq \emptyset$, pois $\tilde{f}^{-1}\left(\Gamma^{+}\right) \cap \Theta=\emptyset$, e, $\widetilde{f}^{-1}\left(\Gamma^{+}\right) \cap \Omega \neq \emptyset$. Se denotamos por $\Gamma^{+*}$ a componente conexa de $\tilde{f}^{-1}\left(\Gamma^{+}\right) \cap \Omega$ que contém $\Gamma$, temos que $\Gamma^{+*}$ está contida em $B^{-}$e, pelo fato de $\widetilde{f}^{-1}\left(\Gamma^{+}\right)$ser conexo, $\Gamma^{+*}$ intercepta $w$, e isso é uma contradição.

Então, $\widetilde{f}(\Gamma)=\Gamma^{+} \subset \Omega$, mas todo o feito acima pode-se aplicar a $\widetilde{f}^{n}(\Gamma)$, pois $\widetilde{f}$ preserva $\prec$, logo, por um argumento inductivo, para todo inteiro positivo $n$ temos que:

- $\tilde{f}^{n}(\Gamma) \cap \Theta=\emptyset$.

- $\tilde{f}^{n}(\Gamma)$ é uma componente conexa de $B^{-}$.

- $\tilde{f}^{n}(\Gamma) \subset \Omega$.

- $\tilde{f}^{n}(\Gamma) \prec \Theta$.

Como $\Gamma$ é uma componente injetora inferior, logo $\widetilde{f}^{n}(\Gamma)$ também, e do Lema 5.4.1 e o fato de $\widetilde{f}$ preservar ordem, temos a afirmação do passo 1 , pois no caso em que $\widetilde{f}(\Gamma)=\Gamma$, pelo fato de $\Gamma$ ser injetora, $\Gamma+(k, 0) \cap \Gamma=\emptyset$, e como ela é uma componente inferior, temos $\Gamma+(k, 0) \prec \Gamma \prec \Theta$. No caso em que $\Gamma^{+} \neq \Gamma$, como $\Gamma^{+}$é injetora $\left(\Gamma^{+}+(k, 0)\right) \cap \Gamma^{+}=\emptyset$, pelo Lema 5.4.1, como antes temos que $\Gamma^{+}+(k, 0) \prec \Gamma^{+}$.

Passo 2: Aqui vamos fazer a mesma construção que no Lema 5.4.2.

Fixemos $k^{\prime}>0$ um inteiro, de forma que $\widetilde{f}(\Gamma)+(k, 0)$ intercepte $w$. Denotemos por $\Gamma^{*}$ a componente conexa ilimitada de $\widetilde{f}(\Gamma)+(k, 0) \cap \bar{\Omega}$. Pela escolha de $k^{\prime}$ e a conexidade de $\widetilde{f}(\Gamma)+\left(k^{\prime}, 0\right)$ temos que $\Gamma^{*}$ não está contida em $B^{-}$, pelo fato dela interceptar $w$. Então, existe um inteiro positivo $a_{1}$ tal que $\widetilde{f}^{a_{1}}\left(\Gamma^{*}\right)$ intercepta $(0,+\infty) \times[0,1]$.

O passo 1 implica que $\widetilde{f}^{a_{1}}(\widetilde{f}(\Gamma)+(k, 0))=\widetilde{f}^{a_{1}+1}(\Gamma)+\left(k^{\prime}, 0\right)$ não intercepta $\Theta$ e é menor que ela na ordem $\prec$. Então, temos que

$$
\Gamma^{*} \subset \widetilde{f}(\Gamma)+\left(k^{\prime}, 0\right) \rightarrow \widetilde{f}^{a_{1}}\left(\Gamma^{*}\right) \cap \Theta=\emptyset \text { e } \widetilde{f}^{a_{1}}\left(\Gamma^{*}\right) \prec \Theta .
$$

Então como antes, temos que pelo fato de $\widetilde{f}^{a_{1}}\left(\Gamma^{*}\right) \cap(0,+\infty) \times[0,1] \neq \emptyset$, implica que $\widetilde{f}^{a_{1}}\left(\Gamma^{*}\right) \cap \Omega$ possui uma componente conexa ilimitada $\Gamma^{* *}$ que intercepta $w$ e portanto não está contida em $B^{-}$, logo existe $a_{2}>0$ tal que $\widetilde{f}^{a_{2}}\left(\Gamma^{*}\right) \subset \widetilde{f}^{a_{2}+a_{1}+1}+\left(k^{\prime}, 0\right)$ intercepta $(0,+\infty) \times[0,1]$. Com o mesmo raciocínio obtemos $\Gamma^{* * *} \subset \widetilde{f}^{a_{2}}\left(\Gamma^{* *}\right) \cap \bar{\Omega}$ que intercepta $w$. Novamente, $\Gamma^{* * *}$ não está contida em $B^{-}$ e existe um inteiro $a_{3}>0$ tal que $\widetilde{f}^{a_{3}}\left(\Gamma^{* * *}\right)$ intercepta $(0,+\infty) \times[0,1]$.

Em geral, definimos $n_{i}=a_{1}+a_{2}+\ldots+a_{i}+1$ e temos que $n_{i} \rightarrow \infty$ se $i \rightarrow \infty$, e para todo $i \geq 1$

$$
\tilde{f}^{n_{i}-1}\left(\tilde{f}\left(\Gamma_{1}\right)+\left(k^{\prime}, 0\right)\right) \supset \widetilde{f}^{n_{i}-1}\left(\Gamma^{*}\right) \supset \widetilde{f}^{a_{2}+\ldots+a_{i}}\left(\widetilde{f}^{a_{i}}\left(\Gamma^{*}\right)\right) \supset \widetilde{f}^{a_{2}+\ldots+a_{i}}\left(\Gamma^{* *}\right) \supset \ldots \supset \widetilde{f}^{a_{i}}\left(\Gamma^{*_{* \ldots *}^{i-\text { veces }}}\right)
$$

E $\widetilde{f}^{a_{i}}(\Gamma \overbrace{}^{i-. * *}) \cap(0,+\infty) \times[0,1] \neq \emptyset$. Logo temos que, por construção

$$
\widetilde{f}^{n_{i}}\left(\Gamma_{1}\right) \cap V_{0}-\left(k^{\prime}, 0\right)=\widetilde{f}^{n_{i}}\left(\Gamma_{1}\right) \cap V_{k^{\prime}} \neq \emptyset
$$

E temos assim provado o Passo 2.

Mas isso implicaría que $\omega\left(B^{-}\right) \neq \emptyset$, contradizendo o Teorema 2. Logo $\Gamma$ só pode ser uma componente não injetora. Provamos assim o Teorema 6. 


\section{Capítulo 6}

\section{$\mathrm{O}$ conjunto $H_{+}^{\text {inst }}$}

Vamos começar, como ao longo do trabalho, lembrando a definição mais importante para este capítulo:

Definição: Seja $H_{+}^{\text {inst }}$ o conjunto dos homeomorfismos que preservam orientação e as componentes de fronteira, e tais que, para qualquer vizinhança $U$ de $\mathbf{S}^{1} \times\{0\}$ e qualquer vizinhança $V$ de $\mathbf{S}^{1} \times\{1\}$, existem inteiros positivos $k_{1}$ e $k_{2}$ tais que $f^{k_{1}}(U) \cap V \neq \emptyset$ e $f^{k_{2}}(V) \cap U \neq \emptyset$.

\subsection{Resultados preliminares}

Aqui, vamos provar que, precisamente $\mathrm{Hom}_{+}^{\text {trans }} \subset H_{+}^{\text {inst }}$, de fato vamos provar algo um pouco mais forte. Notemos que, da definição acima, se $f$ é transitivo, todo ponto de $f$ é não errante, logo, é claro que dada $U$ vizinhança de $\mathbf{S}^{1} \times\{0\}$, existe $\widetilde{U} \subset U$ aberto, e $n_{1}$ inteiro, tal que $f^{n_{1}}(\widetilde{U}) \cap V \neq \emptyset$, agora como todo ponto de $f$ é não errante, existe $\widetilde{U}_{1} \subset f^{n_{1}}\left(\widetilde{U}_{1}\right)$ e $n_{2}$ inteiro positivo (onde eu posso supor $\left|n_{2}\right|>\left|n_{1}\right|$, pois caso contrário repito o proceso) tal que $f^{n_{2}}\left(\widetilde{U}_{1}\right) \cap \widetilde{U}_{1} \neq \emptyset$. Logo, consideramos $\widetilde{U}_{2}=f^{-n_{1}}\left(\widetilde{U}_{1}\right) \subset \widetilde{U} \subset U$ e, como $n_{2}+n_{1}>0$, temos que $f^{n_{2}+n_{1}}\left(\widetilde{U}_{2}\right) \cap V \neq \emptyset$, obtendo assim o procurado. O raciocionio para a outra possibilidade é análogo. Logo mostramos que se $f$ é transitiva no anel então $f \in H_{+}^{\text {inst }}$. Outra cosa para notar, é que a condição de transitividade no anel da como resultado um conjunto maior que $H_{o m}^{\text {trans }}$, pois o fato de ele ser transitivo no anel não implica que tal homeomorfismo possui um levantamento transitivo.

Uma outra coisa para provar aqui é que se $f \in H_{+}^{\text {inst }}$, então $f^{q} \in H_{+}^{\text {inst }}$. Para mostras isso, sejam $U \supset \mathbf{S}^{1} \times\{0\}$ e $V \supset \mathbf{S}^{1} \times\{1\}$, definimos os seguintes conjuntos

$$
\widetilde{U}=\bigcap_{i=0}^{q-1} f^{i}(U) \quad e \quad \widetilde{V}=\bigcap_{i=0}^{q-1} f^{i}(V)
$$

Então temos a seguinte:

Afirmação 6.1.1 : Se $\left(f^{q}\right)^{k}(U) \cap V=\emptyset$ para todo $k$ inteiro positivo, então $f^{N}(\widetilde{U}) \cap \widetilde{V}=\emptyset$ para todo inteiro positivo $N$.

Prova: Certamente, se conseguimos provar está afirmação, concluimos que $f^{q} \in H_{+}^{\text {inst }}$ por contrapositivo, pois, pelo fato de $f \in H_{+}^{\text {inst }}$, a tese de nossa afirmação, é falsa, logo temos o procurado.

A prova vai sair, também, negando a tese. E vamos considerar só o caso em que $q>0$ (no final veremos porque), aqui temos duas possibilidades:

- $q>N$

Então, como existe $N>0$ tal que $f^{N}\left(\bigcap_{i=0}^{q-1} f^{i}(U)\right) \cap \widetilde{V} \neq \emptyset$, temos que $\bigcap_{j=N}^{N+q-1} f^{j}(U) \cap \widetilde{V} \neq \emptyset$, como $N \geq 1,0 \leq q-1<q$ e $N<q$, em algum momento $j=q$, logo, como $\widetilde{V} \cap f^{i}(U) \neq \emptyset$ 
se $j=N, \ldots, N+q-1$, do anterior obtemos que $\widetilde{V} \cap f^{q}(U) \neq \emptyset$, dado que $\widetilde{V}=\bigcap_{i=0}^{q-1} f^{i}(V)$, em particular, $f^{q}(U) \cap V \neq \emptyset$, como o qual, tomando $k=1$ obtemos o contrapositivo.

- $q<N$

Aqui, temos que $N=q \cdot r+l$, com $r, l \geq 0$ e $0 \leq l<q$, por nossa suposição, $f^{N}(\widetilde{U}) \cap \widetilde{V} \neq \emptyset$, notando que

$$
f^{N}(\widetilde{U})=f^{r q}\left(\bigcap_{i=l}^{q-1+l} f^{i}(U)\right)
$$

temos que existe $z \in \bigcap_{i=l}^{q-1+l} f^{i}(U)$ tal que $f^{r q}(z \in \widetilde{V}$. Agora, do fato de $f$ preservar as componentes de fronteira temos que, como $U \supset \mathbf{S}^{1} \times\{0\}, f^{i}(U) \supset \mathbf{S}^{1} \times\{0\}$ para todo $i$ inteiro. Logo, do anterior obtemos que, em particular

$$
U_{1}=\bigcap_{i=-l}^{q-1} f^{i}(U) \subset \bigcap_{i=0}^{q-1} f^{i}(U)=\widetilde{U}
$$

Então, tomando $\widetilde{z} \in U_{1}$, temos que $\widetilde{z} \in U$ e $f^{l}(\widetilde{z}) \in f^{l}\left(U_{1}\right) \subset f^{l}(\widetilde{U})$.

Como $f^{l}(\widetilde{z}) \in f^{l}(\widetilde{U})$, então $f^{r q}\left(f^{l}(\widetilde{z})\right) \in \widetilde{V}$, mas como $\widetilde{z} \in \bigcap_{i=-l}^{q-1} f^{i}(U), f^{l}(\widetilde{z}) \in \bigcap_{i=0}^{q-1+l} f^{i}(U)$, em particular, $f^{l}(\widetilde{z}) \in U$, e temos, trabalhando como acima, que $f^{r q}(U) \cap V=\neq \emptyset$, provando assim o que procuramos.

Notemos que para a prova com $q<0$, a hipóteses da afirmação é o mesmo que peder

$$
\left(f^{|q|}\right)^{-r}(U) \cap V=\emptyset
$$

para todo $r>0$ inteiro, ou, o que é o mesmo

$$
U \cap\left(f^{|q|}\right)^{r}(V)=\emptyset
$$

mas aqui, nossa tesse sería que então $\widetilde{U} \cap f^{N}(\widetilde{V})=\emptyset$, para todo $N>0$, sendo agora que $\widetilde{U}=\bigcap_{i=0}^{|q|-1} f^{i}(U)$ e $\widetilde{V}=\bigcap_{i=0}^{|q|-1} f^{i}(V)$ e isso já está provado no caso anterior, logo concluimos que se $f \in H_{+}^{\text {inst }}$ então $f^{q} \in H_{+}^{\text {inst }}$ para todo inteiro $q$.

Vamos dar a seguinte definição, que vai servir para generalizar os resultados a um conjunto maior de homeomorfismos.

Definição: Seja $f$ um homeomorfismo, dizemos que $f$ tem um levantamento não limitado a esquerda $\widetilde{f}$, se para qualquer $M>0$, existe um ponto $\widetilde{z} \in \widetilde{A}$ e um inteiro positivo $n$ tal que $p_{1}\left(\widetilde{f}^{n}(\widetilde{z})\right)-p_{1}(\widetilde{z})<-M$.

Primeiro, notemos que, claramente, se $f \in H_{o m}^{\text {trans }}$, então $f$ tem um levantamento não limitado a esquerda, pois $f$ tem uma órbita densa, logo tomando $\widetilde{z}$ nessa órbita, onde podemos supor que $p_{1}(\widetilde{z})>0$. Logo, tomamos um conjunto fechado $\widetilde{F}$, cuja distância a $\widetilde{z}$, na primeira componente, seja maior a $M$, e tal que $\widetilde{F}$ à esquerda de $\widetilde{z}$, fazendo o mesmo raciocínio que nos capítulos anteriores, podemos escolher $n>0$ inteiro tal que $\widetilde{f}^{n}(\widetilde{z}) \in \widetilde{F}$ e temos assim o pedido.

Agora temos que, se escolhemos todos os homeomorfismos em $H_{+}^{\text {inst }}$ ilimitados a esquerda, é claro que este conjunto contém $\mathrm{Hom}_{+}^{\text {trans }}$, então agora começaremos a geralizar alguns resultados que já conhecemos do conjunto $\mathrm{Hom}_{+}^{\text {trans }}$ ao conjunto $H_{+}^{\text {inst }}$ que posuim um levantamento não limitado a esquerda.

Notemos que pelo fato de ter que os homeomorfismos em $H_{+}^{\text {inst }}$ preservam a orientação e as componentes de fronteira, podemos como antes, definir os conjuntos $B, B^{-}$e $\omega\left(B^{-}\right)$em $\widetilde{A}$. Começamos 
lembrando que, como antes

$$
B^{-}=\bigcup_{i \in I} \Gamma_{i}
$$

Onde $\Gamma_{i}$ são as componentes conexas ilimitadas do conjunto $B$, e a união é tomada sobre todas as componentes conexas de $B$ ilimitadas.

Para continuar, vamos provar que para os homeomorfismos em $H_{+}^{\text {inst }}$ que possuem um levantamento não limitado a esquerda, o conjunto $B^{-}$é diferente de vazio, e mais ainda, satisfazem o mesmo que os homeomorfismos em $H_{o m}^{\text {trans }}$, isto é que $B^{-} \cap V \neq \emptyset$, temos então que:

Lema 6.1.1 : Seja $f$ um homeomorfismo do anel com um levantamento não limitado a esquerda, que preserva a orientação e as componentes de fronteira. Se os números de rotação de ambas fronteiras são positivos, então $B^{-} \cap V \neq \emptyset$

Demonstração: Notemos duas coisas antes de começar a demonstração, a primeira é que este lema não pede que $f \in H_{+}^{\text {inst }}$, só precisa que o homeomorfismo tenha um levantamento não limitado a esquerda, e a segunda, é que para provar o lema, só provaremos que para qualquer $x$ negativo, existe um $n$ inteiro positivo tal que $\widetilde{f}^{n}(V) \cap V_{x} \neq \emptyset$, logo a prova vai continuar como o Lema 4.1.1 do Capítulo 4.

Mas notemos que, dado que $\tilde{f}$ é não limitada a esquerda, para qualquer $x$ negativo, existe um inteiro positivo $n$ tal que $\widetilde{f}^{n}\left(V^{+}\right) \cap V_{x}^{-} \neq \emptyset$. O anterior sai do fato de considerar que dado qualquer $x$ negativo, só tomando $V_{x}$ e $M>0$ tal que $-M<x-1$, existe $\widetilde{z} \in \widetilde{A}$ e um inteiro positivo $n$ satisfazendo que

$$
p_{1}\left(\widetilde{f}^{n}(\widetilde{z})\right)-p_{1}(\widetilde{z})<-M
$$

Agora, antes de continuar, vamos fazer duas observações, a primeira é que, a hipóteses de $f$ ter um levantamento não limitado a esquerda, implica que existem pontos (pelo menos um) em $\widetilde{A}$ cujas iteradas positivas através de $\widetilde{f}$ ficam a uma distância à esquerda do ponto original tanto como eu queira. A segunda observação é que dado qualquer ponto $\widetilde{z}$, existe um primer inteiro $k$, tal que $\widetilde{z}+(k-1,0) \in V^{-}$e $\widetilde{z}+(k, 0) \in V^{+}$.

Então, tomando $V_{x}, M>0, \widetilde{z} \in \widetilde{A}, n$ inteiro positivo e $k$ como acima, temos que $\widetilde{z}+(k, 0) \in V^{+}$, mais ainda $\widetilde{z}+(k, 0) \in V^{+} \cap V_{1}^{-}$, logo, como $-M<x-1$ e $\tilde{f}$ preserva orientação temos que $\tilde{f}^{n}(\widetilde{z}+(k, 0))$ satisfaz que $p_{1}\left(\tilde{f}^{n}(\widetilde{z}+(k, 0))\right)-p_{1}(\widetilde{z}+(k, 0))<-M$; agora, o anterior fala que $p_{1}\left(\widetilde{f}_{\widetilde{f}}^{n}(k, 0)\right)$ está a uma distância a esquerda de $\widetilde{z}+(k, 0)$ maior a $M$, e temos que, pela escolha de $M, \widetilde{f}^{n}(\widetilde{z}+(k, 0)) \in V_{x}^{-}$, obtendo assim o procurado.

Logo, pelo fto de $\tilde{f}$ presrevar orientação, $\widetilde{f}^{n}(V) \cap V_{x} \neq \emptyset$ e daqui na frente a demostração segue como no Lema 4.1.1 do Capítulo 4.

Agora, definimos o conjunto $\omega\left(B^{-}\right)$como antes, e obtemos novamente o seguinte resultado:

Propriedade: $\omega\left(B^{-}\right)$é um conjunto fechado, $\widetilde{f}$ invariante, cujas componentes conexas são todas ilimitadas.

Até o final da seção, vamos continuar geralizando algumas propriedades satisfeitas pelas $f \in$ $H_{o m}^{\text {trans }}$, e que são satisfeitas também pelos homomorfismos em $H_{+}^{\text {inst }}$.

Lema 6.1.2: $\overline{p\left(B^{-}\right)} \cap\left(\mathbf{S}^{1} \times\{0\} \cup \mathbf{S}^{1} \times\{1\}\right) \neq \emptyset$ e, se $\omega\left(B^{-}\right) \neq \emptyset$, então, $\overline{p\left(\omega\left(B^{-}\right)\right)} \cap\left(\mathbf{S}^{1} \times\{0\} \cup \mathbf{S}^{1} \times\{1\}\right) \neq \emptyset$.

Demonstração: Vamos provar o resultado para $B^{-}$, o outro caso é igual, usando fortemente a invariância de $\omega\left(B^{-}\right)$.

Começamos supondo que o lema é falso. Logo, existe $\epsilon>0$ tal que $p\left(B^{-}\right) \subset \mathbf{S}^{1} \times[\epsilon, 1-\epsilon]$. Como $f \in H_{+}^{\text {inst }}$, existe um ponto $z \in \mathbf{S}^{1} \times[0, \epsilon]$ e um inteiro positivo $n$ tal que $f^{-n}(z) \in \mathbf{S}^{1} \times[1-\epsilon, 1]$.

Agora tomemos $k_{1}$ o segmento ligando $z$ e $\mathbf{S}^{1} \times\{0\}$, e $k_{2}$ o segmento ligando $f^{-n}(z) \operatorname{com} \mathbf{S}^{1} \times\{1\}$. 
$\mathrm{O} \operatorname{arco} \alpha=k_{2} \cup f^{-n}\left(k_{1}\right)$ é uma curva compacta e conexa ligando as duas fronteiras (pois $f$ preserva tais componentes). Logo se $\Gamma$ é qualquer componente conexa não limitada de $\widetilde{A}$, então $p(\Gamma) \cap \alpha \neq \emptyset$.

Agora, tomemos $\Gamma$ uma componente conexa de $B^{-}$, logo por nossa suposição, $p(\Gamma) \cap k_{2}=\emptyset$, portanto, pelo falado acima, obtemos que $p(\Gamma) \cap f^{-n}\left(k_{1}\right) \neq \emptyset$, o que implica que $p\left(\tilde{f}^{n}(\Gamma)\right) \cap k_{1} \neq \emptyset$, e isso é uma contradição, já que $\widetilde{f}^{n}(\Gamma) \subset B^{-}$.

Proposição 6.1.1 : $f\left(p\left(B^{-}\right)\right)=p\left(B^{-}\right)$

Demonstração: Dado que $\widetilde{f}\left(B^{-}\right) \subset B^{-}$, temos que $f\left(p\left(B^{-}\right)\right) \subset p\left(B^{-}\right)$. Agora, vamos mostrar que, se $\widetilde{z} \in B^{-}$, existe um inteiro positivo $i$ tal que $\widetilde{f}^{-1}(\widetilde{z})-(i, 0) \in B^{-}$, isso mostraria que, se $z \in p\left(B^{-}\right)$, existe um $\widetilde{z} \in B^{-}$, tal que $p(\widetilde{z})=z \mathrm{e}$

$$
f^{-1}(z)=f^{-1}(p(\widetilde{z}))=p\left(\widetilde{f}^{-1}(\widetilde{z})\right)=p\left(\tilde{f}^{-1}(\widetilde{z}-(i, 0))\right) \in p\left(B^{-}\right)
$$

acabando assim a prova.

\subsection{Demonstração do Teorema 7}

A idéia da prova e a mesma que antes, começaremos provando que $\omega\left(B^{-}\right)$é um conjunto vazio, e logo que existe um inteiro positivo $N_{1}$ tal que $\widetilde{f}^{N_{1}}\left(B^{-}\right) \subset B^{-}-(1,0)$, como o qual obteremos diretamente o pedido.

Afirmação 6.2.1 $: \omega\left(B^{-}\right)=\emptyset$.

Prova: Suponha, por contradição, que $\omega\left(B^{-}\right) \neq \emptyset$, logo pelo lema 6.1.2, podemos supor que $\overline{p\left(\omega\left(B^{-}\right)\right)} \cap \mathbf{S}^{1} \times\{0\} \neq \emptyset$. Como ambos números de rotação nas fronteiras são positivos, existe $\sigma>0$ tal que se $(\widetilde{x}, i) \in \mathbf{R} \times\{i\}, i=0,1$, então $p_{1}(\widetilde{f}(\widetilde{x}, i))>\widetilde{x}+2 \sigma$, para todo $\widetilde{x}$ real.

Seja $\epsilon>0$ suficientemente pequeno tal que para todo $(\widetilde{x}, \widetilde{y}) \in \mathbf{R} \times[0, \epsilon]$ e para todo $(\widetilde{x}, \widetilde{y}) \epsilon$ $\mathbf{R} \times[1-\epsilon, 1]$ seja satisfeito que $p_{1} \circ \widetilde{f}(\widetilde{x}, \widetilde{y})>\widetilde{x}+\sigma$ (essa escolha é posível pois $\widetilde{f}$ é continua). Pelo provado em capitulos anteriores existe um número real $a<0$, tal que $\omega\left(B^{-}\right) \cap\{a\} \times[0, \epsilon] \neq \emptyset$.

Do fato de $\omega\left(B^{-}\right)$ser fechado, existe $0<\delta \leq \epsilon$ tal que o ponto $(a, \delta) \in \omega\left(B^{-}\right)$e tal que, para todo $\widetilde{y}, 0 \leq \widetilde{y}<\delta,(a, \widetilde{y}) \notin \omega\left(B^{-}\right)$, ou seja, $(a, \delta)$ é o ponto mais baixo de $\omega\left(B^{-}\right)$no segmento $\{a\} \times[0, \epsilon]$. Vamos denotar por $v$ ao segmento $\{a\} \times[0, \delta)$ (note que $\left.\omega\left(B^{-}\right) \cap v=\emptyset\right)$.

Tomemos $\Theta_{1}$ a componente conexa não limitada de $\omega\left(B^{-}\right)$que contém $(a, \delta)$, e seja $\Omega_{1}$ a componente conexa de $\left(\Theta_{1} \cup v\right)^{c}$ que contém $(-\infty, a) \times\{0\}$.

Pela construção, $\partial \Omega_{\tilde{1}} \subset \Theta_{1} \cup v$, e pelo fato de $\widetilde{f}$ ser homeomorfismo e $\left(\Theta_{1} \cup v\right)$ fechado, temos que $\partial \widetilde{f}\left(\Omega_{1}\right) \subset \widetilde{f}\left(\Theta_{1}\right) \cup \widetilde{f}(v)$.

Além disso, dado que $\omega\left(B^{-}\right) \cap v=\emptyset$ e $\omega\left(B^{-}\right)$é $\widetilde{f}$ invariante temos que:

- $\widetilde{f}\left(\Theta_{1}\right) \cap v=\emptyset$ pois $\widetilde{f}\left(\Theta_{1}\right) \subset \omega\left(B^{-}\right)$(e, em geral, pelo mesmo motivo $\left.\widetilde{f}\left(\omega\left(B^{-}\right)\right) \cap v=\emptyset\right)$.

- $\Theta_{1} \cap \tilde{f}(v)=\emptyset$ pois caso contrário, se $z \in \Theta_{1} \cap \tilde{f}(v)$ pela invariância de $\omega\left(B^{-}\right)$teríamos uma contradição.

- $\widetilde{f}(v) \cap v=\emptyset$ pela escolha do $\epsilon$.

Então, dado que $\left(\Theta_{1} \cup v\right)$ é fechado, temos que $\Omega_{1}$ é aberto, o que vamos provar agora é que $\Omega_{1} \subset \widetilde{f}\left(\Omega_{1}\right)$.

Observemos que, pelo falado acima, temos $\partial \widetilde{f}\left(\Omega_{1}\right) \subset \omega\left(B^{-}\right) \cup \widetilde{f}(v)$, e pela hipótese acerca do número de rotação de $\widetilde{f}$ na fronteira $(-\infty, a) \times\{0\} \subset \widetilde{f}((-\infty, a) \times\{0\}) \subset \widetilde{f}\left(\Omega_{1}\right), \operatorname{logo} \Omega_{1} \cap \widetilde{f}\left(\Omega_{1}\right) \neq \emptyset$.

Logo temos duas possibilidades (por ser $\omega\left(B^{-}\right)$união de componentes conexas e $\widetilde{f}$ invariante):

- $\tilde{f}\left(\Theta_{1}\right) \cap \Theta_{1}=\emptyset$ 
- $\tilde{f}\left(\Theta_{1}\right)=\Theta_{1}$

Se começamos assumindo que $\widetilde{f}\left(\Theta_{1}\right) \cap \Theta_{1}=\emptyset$, como $\Theta_{1} \cup v$ é conexo, pelo falado acima temos que $\widetilde{f}\left(\Theta_{1} \cup v\right) \cap\left(\Theta_{1} \cup v\right)=\emptyset$ e $\partial \Omega_{1} \subset \Theta_{1} \cup v$, segue-se que $\widetilde{f}\left(\Theta_{1} \cup v\right) \cap \partial \Omega_{1}=$.

Agora, como $(a, 0) \in v$ e $\widetilde{f}((a, 0)) \notin \Omega_{1}$, e $\Theta_{1} \cup v$ é conexo, pelo falado acima temos que $\tilde{f}\left(\Theta_{1} \cup v\right) \cap \Omega_{1}=\emptyset$. Como $\partial \tilde{f}\left(\Omega_{1}\right) \subset \widetilde{f}\left(\Theta_{1} \cup v\right)$, temos, pela afirmação 3.1.2, que $\Omega_{1} \subset \widetilde{f}\left(\Omega_{1}\right)$.

Agora, se asumimos que $\widetilde{f}\left(\Theta_{1}\right)=\Theta_{1}$, como $\Theta_{1} \cap \Omega_{1}=\emptyset$ e $v \cap \Omega_{1}=\emptyset$, claramente $\partial \widetilde{f}\left(\Omega_{1}\right) \cap \Omega_{1}=\emptyset$ e outra vez, pela escolha do $v$ e a afirmação 3.1.2, temos que $\Omega_{1} \subset \widetilde{f}\left(\Omega_{1}\right)$.

Agora, $\Omega_{1}$ é aberto e contém $[a-2, a-1] \times\{0\}$, logo, como $\widetilde{A}$ é o recobrimento universal, e $p((\widetilde{x}+1, \widetilde{y}))=p((\widetilde{x}, \widetilde{y}))$, para qualquer $(\widetilde{x}, \widetilde{y}) \in \widetilde{A}$, temos que $p\left(\Omega_{1}\right)$ é uma vizinhançade $\mathbf{S}^{1} \times\{0\}$, e dado que $\Omega_{1} \subset \widetilde{f}\left(\Omega_{1}\right)$, segue-se que $p\left(\Omega_{1}\right) \subset f\left(p\left(\Omega_{1}\right)\right)$.

Como $f \in H_{+}^{\text {inst }}$, existe uma seqüência de pontos $x_{n}$, arbitrariamente perto de $\mathbf{S}^{1} \times\{1\}$ cujas órbitas positivas, em algum momento estão em $p\left(\Omega_{1}\right)$, mas a invariância negativa de $\Omega_{1}$ (se $\Omega_{1} \subset \widetilde{f}\left(\Omega_{1}\right)$ então $\left.\widetilde{f}^{-1}\left(\Omega_{1}\right) \subset \Omega_{1}\right)$ mostra que $x_{n} \in p\left(\Omega_{1}\right)$, pois $f^{-k}\left(p\left(\Omega_{1}\right)\right) \subset p\left(\Omega_{1}\right)$ para qualquer inteiro positivo $k$. Por tudo feito até agora, $p\left(\Omega_{1}\right)$ intercepta qualquer vizinhança de $\mathbf{S}^{1} \times\{1\}$, o que implica que $d\left(\Omega_{1} ; \mathbf{R} \times\{1\}\right)=0$. Como $\partial \Omega_{1} \subset \Theta_{1} \cup v$ e dado que, claramente $d(v ; \mathbf{R} \times\{1\})>0$ temos que $d\left(\Theta_{1} ; \mathbf{R} \times\{1\}\right)=0$, segue-se que $\overline{p\left(\omega\left(B^{-}\right)\right)} \cap \mathbf{S}^{1} \times\{1\} \neq \emptyset$.

Agora, provado o anterior, podemos trabalhar como antes na construção de $\Omega_{1}$ mas em $\mathbf{S}^{1} \times\{1\}$, logo, pelo provado acima, existe $b<0$, e um ponto $\left(b, 1-\delta_{2}\right)$, com $0<\delta_{2}<\epsilon$, tal que $\left(b, 1-\delta_{2}\right) \in$ $\omega\left(B^{-}\right)$e tal que se $w=\{b\} \times\left(1-\delta_{2}, 1\right]$ então $w \cap \omega\left(B^{-}\right)=\emptyset$, e tomamos $\Theta_{2}$ a componente conexa de $\omega\left(B^{-}\right)$que contém $\left(b, 1-\delta_{2}\right)$ e chamamos $\Omega_{2}$ à componente conexa de $\left(\Theta_{2} \cup w\right)^{c}$ que contém $(-\infty, b) \times\{1\}$. Dado que a construção é a mesma, obtemos novamente que $\Omega_{2} \subset \widetilde{f}\left(\Omega_{2}\right)$.

Consideramos agora o conjunto:

$$
\Omega_{3}=\left(\bigcup_{i=0}^{\infty} \Omega_{1}-(i, 0)\right) \cup\left(\bigcup_{i=0}^{\infty} \Omega_{2}-(i, 0)\right)
$$

então temos a seguinte:

Proposição 6.2.1 : $\Omega_{3}$ é conexo.

Demonstração: Primeiro, notemos que, como $\Omega_{1}$ é conexo e como $(-\infty, a-i) \times\{0\} \subset\left(\Omega_{1} \cap \Omega_{1}-(i, 0)\right)$ para cada $i$ inteiro positivo, isso implica que $\left(\bigcup_{i=0}^{\infty} \Omega_{1}-(i, 0)\right)$ é um conjunto aberto e conexo. Pelo mesmo motivo $\left(\bigcup_{i=0}^{\infty} \Omega_{2}-(i, 0)\right)$ é aberto e conexo.

Como $\Omega_{2}$ é aberto e $[b-2, b-1] \times\{1\} \subset \Omega_{2}$. existe um $\delta_{3}>0$ tal que $[b-2, b-1] \times\left[1-\delta_{3}, 1\right] \subset$ $\Omega_{2}$ implicando que

$$
[-\infty, b-1] \times\left[1-\delta_{3}, 1\right] \subset\left(\bigcup_{i=0}^{\infty} \Omega_{2}-(i, 0)\right)
$$

Notemos que, como $\overline{p\left(\Omega_{1}\right)} \cap \mathbf{S}^{1} \times\{1\} \neq \emptyset$, podemos achar um ponto $\left(c, 1-\delta_{4}\right) \in \Omega_{1}$, onde $\delta_{4}<\delta_{3}$, logo, por (2.1), existe um inteiro positivo $i$ tal que $\left(c-i, 1-\delta_{4}\right) \in(-\infty, b-1) \times\left[1-\delta_{3}, 1\right]$, então temos que $\left(c-i, 1-\delta_{4}\right) \in \Omega_{1}-(i, 0)$ e $\left(c-i, 1-\delta_{4}\right) \in \bigcup_{i=0}^{\infty} \Omega_{2}-(i, 0)$, isso mostra que $\left(\bigcup_{i=0}^{\infty} \Omega_{1}-(i, 0)\right) \cap\left(\bigcup_{i=0}^{\infty} \Omega_{2}-(i, 0)\right) \neq \emptyset$ dando como resultado que $\Omega_{3}$ é conexo, como queriamos provar.

Agora, como $\Omega_{3}$ é aberto e conexo, existe um arco continuo $\alpha$ ligando os pontos $(a-1,0)$ e $(b-1,0)$, com $\alpha \subset \Omega_{3}$. Como por construção, $\Omega_{3} \subset \widetilde{f}\left(\Omega_{3}\right)$ e $V \cap \Omega_{3}=\emptyset$, segue-se que $\widetilde{f}^{n}(V) \cap \alpha=\emptyset$ para todo $n$ inteiro positivo (pela invariância negativa do $\Omega_{3}$ ). Mas o anterior contradiz o fato de $\widetilde{f}$ ser não limitada a esquerda já que, como $\alpha$ é um arco continuo, existe um $x$ negativo, suficientemente pequeno tal que $\alpha \subset V_{x}^{+}$, e fazendo a mesma construção que no Lema 6.1.1, teríamos que $\widetilde{f}^{n}(V) \cap V_{x} \neq \emptyset$ para algum $n$ inteiro positivo, e como os numeros de rotação nas fronteiras são estritamente positivos, temos que $\tilde{f}^{n}(V) \cap \alpha \neq \emptyset$, mas isso provamos que não pode acontecer, logo temos um contradição, que decorre de supor que $\omega\left(B^{-}\right) \neq \emptyset$, logo provamos nossa afirmação. 
Agora estamos nas mesmas condições do Lema 4.4.1, usando esse Lema e o resultado de [1], temos provado o Teorema 7 .

\subsection{Demonstração do Corolário 2}

Primeiro, vamos mostrar que para todo ponto $z \in \widetilde{A}$ se satisfaz que $\left|p_{1}(\widetilde{f}(z))-p_{1}(z)\right|<L$, para $\operatorname{algum} L>0$ fixo. Para mostrar isso, só basta considerar $z \in[0,1] \times[0,1]$, já que para qualquer otro ponto em $\widetilde{A}$ é da forma $z+(p, 0)$, com $p$ inteiro, logo se o anterior fora certo, temos que

$$
\left|p_{1}\left(\widetilde{f}^{i}(z+(p, 0))\right)-p_{1}(z+(p, 0))\right|=\left|p_{1}\left(\widetilde{f}^{i}(z+(p, 0))\right)-p_{1}(z)-p\right|=\left|p_{1}(\widetilde{f}(z))-p_{1}(z)\right|<L
$$

pelo fato de $\tilde{f}$ preservar orientação.

Mas o fato de que o $L$ exista sai do fato de que $\widetilde{f}$ é continua, $p_{1}$ é continua, a função diferença é uma função contínua, e a função norma também o é, $\operatorname{logo} h(z)=\left|p_{1}\left(f^{i}(z)\right)-p_{1}(z)\right|$ é uma função contínua definida sobre um compacto, logo ela é limitada, provando assim, que

$$
\left|p_{1}(\widetilde{f}(z))-p_{1}(z)\right|<L
$$

para algum $\mathrm{L}>0$ fixo e para todo $z \in \widetilde{A}$.

Agora suponha que o corolario é falso, logo, para todo $M>0$, existe $z \in \widetilde{A}$ e um inteiro positivo $n$ tal que

$$
p_{1}\left(\tilde{f}^{n}(z)\right)-p_{1}(z)-a n<-M
$$

Notemos que, como estamos supondo $a$ racional, $a=p / q$, com $p$ e $q$ inteiros e eu vou supor $q>0$ (se $a<0, p<0$ ).

Vamos definir $g=f^{q}$ e $\widetilde{g}=\widetilde{f}^{q}-(p, 0)$, agora, para definir $\rho(\widetilde{g})$, lembremos que é definido como todos os pontos de acumulação do conjunto

$$
\left\{\frac{p_{1}\left(\widetilde{g}^{n}(z)\right)-p_{1}(z)}{n} ; z \in \widetilde{A} ; n \in \mathbf{N}\right\}
$$

$\underset{\widetilde{A}}{\operatorname{mas}}$ isso é equivalente a pedir que exista uma seqüência $\left\{n_{k}\right\} \subset \mathbf{N}$ e $\left\{z_{k}\right\}$ seqüência de pontos em $\widetilde{A}$ tal que

$$
\lim _{n_{k} \rightarrow \infty} \frac{p_{1}\left(\widetilde{g}^{n_{k}}\left(z_{k}\right)\right)-p_{1}\left(z_{k}\right)}{n_{k}}=r
$$

para algum $r \in \mathbf{R}$, mas

$$
\frac{p_{1}\left(\widetilde{g}^{n_{k}}\left(z_{k}\right)\right)-p_{1}\left(z_{k}\right)}{n_{k}}=\frac{p_{1}\left(\left(\tilde{f}^{q}-(p, 0)\right)^{n_{k}}\left(z_{k}\right)\right)-p_{1}\left(z_{k}\right)}{n_{k}}
$$

Logo, lebrando que $\tilde{f}$ preserva orientação, temos, pelas mesmas contas que antes que (5.2) fica

$$
\frac{p_{1}\left(\left(\tilde{f}^{q}\right)^{n_{k}}\left(z_{k}\right)-\left(n_{k} p, 0\right)\right)-p_{1}\left(z_{k}\right)}{n_{k}}=\frac{p_{1}\left(\tilde{f}^{q n_{k}}\left(z_{k}\right)\right)-p_{1}\left(z_{k}\right)}{n_{k}}-p
$$

ou, o que é o mesmo

$$
q \frac{p_{1}\left(\widetilde{f}^{q n_{k}}\left(z_{k}\right)\right)-p_{1}\left(z_{k}\right)}{q n_{k}}-p
$$

Então, do anterior obtemos que, se $\rho(\widetilde{f})=[a, b]$ temos que

$$
\rho(\widetilde{g})=[0, q b-p]
$$


Pelo já provado, $g \in H_{+}^{\text {inst }}$, agora, a idéia é provar, supondo o corolário falso, que $g$ possui um levantamento ilimitado a esquerda (claramente, pelas hipóteses do corolario, os numeros de rotação nas fronteiras de $\widetilde{g}$ são estritamente maiores que zero), logo, provando isso, teríamos, pelo Teorema anterior, que 0 está no interior de $\rho(\widetilde{g})$ e isso é uma contradição, pois acabamos de provar que 0 é um extremo de $\rho(\widetilde{g})$. Logo temos que o corolario é verdadero.

Então, como estamos supondo o corolario falso, existem seqüências $\left\{M_{k}\right\} \subset \mathbf{N} ;\left\{z_{k}\right\} \subset \widetilde{A}$ e $\left\{n_{k}\right\} \subset \mathbf{N}$ tais que, $M_{k} \rightarrow \infty$ se $k \rightarrow \infty$, e por continuidade de $\widetilde{f}, n_{k} \rightarrow \infty$ quando $k \rightarrow \infty$ e

$$
p_{1}\left(\tilde{f}^{n_{k}}\right)\left(z_{k}\right)-p_{1}\left(z_{k}\right)-\frac{p}{q} n_{k}<-M_{k}
$$

Notemos que podemos considerar, sem perda de generalidade, que $n_{k}=r_{k} q+l_{k}$ com $0 \leq l_{k}<q$ e $r_{k}>0$, ambos inteiros. Logo $n_{k} \rightarrow \infty$ se e só se $r_{k} \rightarrow \infty$. Então o anterior pode ser escrito como

$$
p_{1}\left(\tilde{f}^{r} q\left(\widetilde{f}^{l_{k}}\left(z_{k}\right)\right)\right)-p_{1}\left(z_{k}\right)-p r_{k}-\frac{p}{q} l_{k}<-M_{k}
$$

chamando $\widetilde{z}_{k}=\widetilde{f}^{l}{ }_{k}\left(z_{k}\right)$ temos

$$
p_{1}\left(\widetilde{g}^{r_{k}}\left(\widetilde{z}_{k}\right)-p_{1}\left(z_{k}\right)-\frac{p}{q} l_{k}<-M_{k}\right.
$$

sumando e sustraindo $p_{1}\left(\widetilde{z}_{k}\right)=p_{1}\left(\widetilde{f}^{l}\left(z_{k}\right)\right)$ obtemos

$$
p_{1}\left(\widetilde{g}^{r_{k}}\left(\widetilde{z}_{k}\right)\right)-p_{1}\left(\widetilde{z}_{k}\right)+p_{1}\left(\widetilde{f}^{l_{k}}\left(z_{k}\right)\right)-p_{1}\left(z_{k}\right)-\frac{p}{q} l_{k}<-M_{k}
$$

logo temos, por todo o feito até agora nesta demonstração que

$$
p_{1}\left(\widetilde{g}^{r_{k}}\left(\widetilde{z_{k}}\right)-p_{1}\left(\widetilde{z}_{k}\right)<-M_{k}-\left(p_{1}\left(\widetilde{f}^{l_{k}}\left(z_{k}\right)\right)-p_{1}\left(z_{k}\right)+\frac{p}{q} l_{k}<-M_{k}-L+\frac{p}{q} l_{k}\right.\right.
$$

Observemos que como $0 \leq l_{k}<q$, o $\frac{p}{q} l_{k}$ é um valor limitado, como $L$ é fixo, temos que $-M_{k}-L+$ $\frac{p}{q} l_{k} \rightarrow \infty$ quando $k \rightarrow \infty$, com o qual, $g$ possui um levantamento ilimitado a esquerda, como o qual provamos que 0 está no interior do conjunto de rotação de $\widetilde{g}$, que era o que buscabamos provar. Logo pelo falado antes, este resultado prova o Corolario.

\subsection{Demonstração do Teorema 8}

Neste capitulo, começarems mostrando alguns resultados preliminares, feito isso, a prova dos resultados principais será conseqüência destas provas.

Lema 6.4.1 : Toda componente conexa de ${\overline{\left(p\left(B^{-}\right)\right)}}^{C}$ é homotópicamente trivial.

Demonstração: Vamos assumir, por contradição que ${\overline{\left(p\left(B^{-}\right)\right)}}^{C}=E$, e que existe uma curva fechada simples, homotópicamente não trivial $\gamma_{E}$ no interior de $E$. Claramente, o conjunto $\gamma_{E}^{C}$ possui duas componentes conexas, uma que contém $\mathbf{S}^{1} \times\{0\}$, denotada por $\gamma_{E}^{-}$, e outra que contém $\mathbf{S}^{1} \times\{1\}$, denotada por $\gamma_{E}^{+}$.

Observemos que, se $\Gamma$ é uma componente conexa de $B^{-}$, então ou $p(\Gamma) \subset \gamma_{E}^{-}$ou $p(\Gamma) \subset \gamma_{E}^{+}$. Agora como (pelo Lema 6.1.2) $\overline{p\left(B^{-}\right)} \cap\left(\mathbf{S}^{1} \times\{0\} \cup \mathbf{S}^{1} \times\{1\}\right) \neq \emptyset$, podemos assumir, sem perda de generalidade, que $\overline{p\left(B^{-}\right)} \cap \mathbf{S}^{1} \times\{0\} \neq \emptyset$. Tomemos também $\epsilon>0$ suficientemente pequeno tal que

- $\mathbf{S}^{1} \times[0, \epsilon] \subset \gamma_{E}^{-}$e $\mathbf{S}^{1} \times[1-\epsilon, 1] \subset \gamma_{E}^{+}$.

- Para todo $(\widetilde{x}, \widetilde{y}) \in \mathbf{R} \times([0, \epsilon] \cup[1-\epsilon, 1])$, vale $p_{1}(\widetilde{f}(\widetilde{x}, \widetilde{y}))>\widetilde{x}$. 
Agora vamos fazer uma construção similar à feita na demonstração do Teorema 1 , obtendo assim um ponto $(a, \delta) \in B^{-}$, tal que $\delta<\epsilon$ tal que, se $v=a \times[0, \delta)$, então $v \cap B^{-}=\emptyset$. Seja $\Gamma_{1}$ a componente conexa de $B^{-}$que contém $(a, \delta)$, e $\Omega$ a componente conexa de $\left(\Gamma_{1} \cup v\right)^{C}$ que contém $(-\infty, a] \times\{0\}$.

Pela escolha do $\epsilon$, temos que $v \subset \gamma_{E}^{-}$, e como $\Gamma_{1} \cup v$ é conexo, $\partial \Omega \subset \Gamma_{1} \cup v$ e $p\left(\Gamma_{1}\right) \cap \gamma_{E}=\emptyset$, isso mostra que $p(\partial \Omega) \subset \gamma_{E}^{-}$e portanto temos que $p(\Omega) \subset \gamma_{E}^{-}$.

Proposição 6.4.1 : $\tilde{f}^{-1}(v) \subset \Omega$.

Demonstração: Notemos que $v \cap B^{-}=\emptyset$, e como $\widetilde{f}\left(\Gamma_{1}\right) \subset B^{-}$, então

$$
\widetilde{f}\left(\Gamma_{1}\right) \cap v=\Gamma_{1} \cap \widetilde{f}^{-1}(v)=\emptyset
$$

Além disso, pela escolha do $\epsilon, \widetilde{f}(v) \cap v=\emptyset$ e também $\tilde{f}^{-1}(v) \cap v=\emptyset$. Como $\partial \Omega \subset \Gamma_{1} \cup v$, temos que $\widetilde{f}^{-1}(v) \cap \partial \Omega=\emptyset$. Como $\widetilde{f}^{-1}(v)$ é conexo, e $\widetilde{f}^{-1}((a, 0)) \in \Omega \cap \widetilde{f}^{-1}(v)$ concluimos que $\widetilde{f}^{-1}(v) \subset \Omega$, provando assim a proposição.

Proposição 6.4.2 : Para todo inteiro $n \geq 0, p\left(\bigcup_{i=0}^{n} \tilde{f}^{-i}(\Omega)\right) \subset \gamma_{E}^{-}$

Demonstração: Vamos provar a proposição por indução. Já sabemos que vale para $n=1$, suponha agora que o resultado é valido para $n=k-1$. Da proposição anterior, obtemos que $\widetilde{f}^{-k}(v) \subset \widetilde{f}^{-k+1}(\Omega)$, e como $p\left(\widetilde{f}^{-k+1}(\Omega)\right) \subset \gamma_{E}^{-}$(argumento indutivo), temos que $p\left(\widetilde{f}^{-k}(v)\right) \subset \gamma_{E}^{-}$.

Notemos que $\widetilde{f}^{-k}\left(\Gamma_{1}\right) \cup \widetilde{f}^{-k}(v)$ é conexo e como $\Gamma_{1} \subset B^{-}$, dos resultados preliminares temos que $p\left(\widetilde{f}^{-k}\left(\Gamma_{1}\right)\right) \subset p\left(B^{-}\right)$, e portanto $p\left(\widetilde{f}^{-k}\left(\Gamma_{1}\right)\right) \subset \gamma_{E}^{-}$(isso é claro, pois $\widetilde{f}^{-k}(a, \delta) \in \widetilde{f}^{-k}\left(\Gamma_{1}\right) \cap \widetilde{f}^{-k}(v) \mathrm{e}$ $\widetilde{f}^{-k}(a, \delta) \in \gamma_{E}^{-}$, como as componentes conexas de $p\left(B^{-}\right)$moram ou $\gamma_{E}^{-}$ou em $\gamma_{E}^{+}$temos o resultado)

Todo o anterior implica que $p\left(\partial \widetilde{f}^{-k}(\Omega)\right) \subset \gamma_{E}^{-}$, lo que da como resultado que $p\left(\tilde{f}^{-k}(\Omega)\right) \subset \gamma_{E}^{-}$, como queria demonstrar.

Seja agora o conjunto

$$
\Omega_{1}=\bigcup_{i=0}^{\infty} \tilde{f}^{-i}(\Omega)
$$

Pela definição do conjunto, é claro que $\Omega_{1} \subset \widetilde{f}\left(\Omega_{1}\right)$, e também é claro que $p\left(\Omega_{1}\right)$ é uma vizinhança de $\mathbf{S}^{1} \times\{0\}$ pois $p(\Omega)$ o é, mas pelo provado acima temos que

$$
p\left(\Omega_{1}\right)=\bigcup_{i=0}^{\infty} p\left(\tilde{f}^{-i}(\Omega)\right) \subset \gamma_{E}^{-}
$$

O que, pela invariância negativa de $p\left(\Omega_{1}\right)$, como antes, contradiz o fato de estar $f \in H_{+}^{\text {inst }}$. Assim, provamos o Lema 6.4.1.

Notemos que, dado $\epsilon>0$, existe um racional $p / q$ tal que $a<p / q<a+\epsilon$ e tal que os numeros de rotação das duas fronteiras é maior que $p / q$. Seja agora $g=f^{q}$ e $\widetilde{g}=\widetilde{f}^{p}-(p, 0)$. Dado que $f \in H_{+}^{\text {inst }}$, segue que $f^{q} \in H_{+}^{\text {inst }}$, e os numeros de rotação das fronteiras são estritamente positivos para $\widetilde{g}$. Construimos então, da mesma forma que antes, o conjunto $B_{g}^{-}$. O Teorema 1 mostra que, para todo ponto $\widetilde{z} \in B_{g}^{-}$, temos que

$$
0>\limsup _{n \rightarrow \infty} \frac{p_{1}\left(\widetilde{g}^{n}(\widetilde{z})\right)-p_{1}(\widetilde{z})}{n}=\limsup _{n \rightarrow \infty} \frac{p_{1}\left(\widetilde{f}^{n q}(\widetilde{z})\right)-p_{1}(\widetilde{z})}{n}-p
$$


e então pela mesma conta feita na demonstração do Corolario anterior

$$
\limsup _{n \rightarrow \infty} \frac{p_{1}\left(\widetilde{f}^{n}(\widetilde{z})\right)-p_{1}(\widetilde{z})}{n}<p / q
$$

Daí concluimos que $B_{g}^{-} \subset K_{\epsilon}, \operatorname{logo} \overline{B_{g}^{-}} \subset K_{\epsilon}$, e portanto $\left(\overline{B_{g}^{-}}\right)^{C} \supset K_{\epsilon}^{C}$, com o qual, o Teorema 8 segue do Lema anterior.

\subsection{Demonstração do Corolário 3}

Lembremos que os conjuntos $K_{\epsilon}$ e $K_{\epsilon}^{C}$ são conjuntos $f$-invariantes. Agora seja $E$ uma componente conexa de $K_{\epsilon}^{C}$, e $\widetilde{E} \subset \widetilde{A}$ uma componente conexa de $p^{-1}\left(K_{\epsilon}^{C}\right)$ que satisfaz $p(\widetilde{E})=E$, se $E$ possui um ponto não errante $z$ (lembremos que o conjunto dos pontos não errantes contém ao conjunto dos pontos recurrentes), , existe um inteiro $q$ tal que $f^{q}(E) \cap E \neq \emptyset$ (eu suponho que $q$ é o primeiro inteiro para o qual isso acontece), como cada componente conexa de $K_{\epsilon}^{C}$ é homotópicamente trivial, temos que $\widetilde{E} \cap \widetilde{E}+(i, 0)=\emptyset$ para todo inteiro $i$. Agora como $K_{\epsilon}^{C}$ é invariante, o fato de ter $f^{q}(E) \cap E \neq \emptyset$ implica que $f^{q}(E)=E$, logo, existe um inteiro $p$ tal que $\widetilde{f}^{q}(\widetilde{E})=\widetilde{E}+(p, 0)$.

Suponha agora que $z \in R_{f} \cap K_{\epsilon}^{C}$, e que $z$ tivesse número de rotação. Como $z$ é um ponto recurrente, existe uma seqüência $n_{k}$ tal que $\lim _{n_{k} \rightarrow \infty} f^{n_{k}}(z)=z$, pelo falado acima, $n_{k}$ tem que ser da forma $n_{k}=r_{k} q\left(n_{k} \rightarrow \infty\right.$ se e só se $\left.r_{k} \rightarrow \infty\right)$.

Logo

$$
z=\lim _{n_{k} \rightarrow \infty} f^{n_{k}}(z)=\lim _{r_{k} \rightarrow \infty}\left(f^{q}\right)^{r_{k}}(z)
$$

como estou supondo que $\widetilde{f}^{q}(\widetilde{E})=\widetilde{E}+(p, 0)$ temos que $\widetilde{f}^{r}{ }_{k} q(\widetilde{E})=\widetilde{E}+\left(r_{k} p, 0\right)$. Como $z$ tem numero de rotação, o limite

$$
\lim _{n \rightarrow \infty} \frac{p_{1}\left(\widetilde{f}^{n}(z)\right)-p_{1}(z)}{n}
$$

existe, em particular existe o seguinte

$$
\lim _{r_{k} \rightarrow \infty} \frac{p_{1}\left(\widetilde{f}^{r} q(z)\right)-p_{1}(z)}{r_{k} q}
$$

e são iguais, mas

$$
\lim _{r_{k} \rightarrow \infty} \frac{p_{1}\left(\widetilde{f}^{r_{k}} q(z)\right)-p_{1}(z)}{r_{k} q}=\lim _{r_{k} \rightarrow \infty} \frac{p_{1}\left(\widetilde{f}^{n_{k}}(z)\right)+p r_{k}-p_{1}(z)}{r_{k} q}=\lim _{r_{k} \rightarrow \infty} \frac{p_{1}\left(\widetilde{f}^{r_{k}} q(z)\right)-p_{1}(z)}{r_{k} q}+\frac{p}{q}=\frac{p}{q}
$$

Logo, pela unicidade do limite, o numero de rotação de $z$ é $p / q$ e provamos assim a primeira parte do corolario.

Para a segunda parte do corolario, notemos que se $f$ é transitiva, ela possui uma órbita densa, como $K_{\epsilon}$ é invariante, se ele contém um ponto de tal órbita, ele contém toda a órbita e, pelo fato de ele ser fechado, temos que $K_{\epsilon}=A$. Se ele não contém a órbita densa, claramente ele tem interior vazio, pois caso contrário em algum momento, algum ponto da órbita densa estaría no interior de $K_{\epsilon}$, e isso não acontece.

Agora, se $K_{\epsilon} \neq A$, e $f$ é transitiva temos que, se $E$ é uma componente conexa de $K_{\epsilon}^{C}$, como antes obtemos um primer inteiro positivo $q$, logo isso falaría que existem exactamente $q$ componentes conexas diferentes de $K_{\epsilon}^{C}$, pois claramente $f^{i}(E) \cap E=\emptyset$ se $i=1, \ldots, q-1$, e além disso $f^{i}(E) \cap f^{j}(E)=\emptyset$ para qualquer $i \neq j, 1 \leq i, j \leq q-1$, pois caso contrário suponha $i<j$ e $f^{i}(E) \cap f^{j}(E) \neq \emptyset, \log 0 j-i<q$ e $E \cap f^{j-i}(E) \neq \emptyset$ o qual não acontece. Agora suponha que eu tivesse $q+1$ componentes conexas diferentes em $K_{\epsilon}^{C}$ (a prova para uma quantidade maior sai por indução). Seja $E_{q+1}$ a componente conexa que não é imagem de $f^{i}(E) \operatorname{com} i=1, \ldots, q-1$. Como $K_{\epsilon}^{C}$ contém a órbita densa, existe $z_{0} \in E$ e $n_{0}$ inteiro positivo, tal que $f^{n_{0}}\left(z_{0}\right) \in E_{q+1}$. Agora notemos 
dos coisas, primeiro, claramente $n_{0}=r i$ para algum $i$ tal que $1 \leq i \leq q$, e a segunda é que se $E$ é $f^{q}{ }_{-}$ invariante, $f^{i}(E)$ também é $f^{q}$-invariante para $i=1, \ldots, q-1$ pois $f^{q}\left(f^{i}(E)\right)=f^{i}\left(f^{q}(E)\right)=f^{i}(E)$. Agora como estamos supondo que $f^{n_{0}}\left(z_{0}\right) \in E_{q+1}$ então $n_{0}>q$, portanto $n_{0}=r q+i$ com $0 \leq i<q$. Também, notemos que se $E$ é $f^{q}$-invariante, é $f^{q r}$-invariante, logo todo o anterior implicaría que $E_{q+1} \cap f^{i}(E) \neq \emptyset$ o qual é uma contradição, que prova que existem exactamente $q$ componentes conexas em $K_{\epsilon}^{C}$. Pelo provado no começo da demonstração concluimos a demonstração do corolario.

\subsection{Demonstração do Corolário 4}

Se $K_{\epsilon} \neq A$, como $f$ preserva a medida de lebesgue, quase todo ponto em $A$ tem numero de rotação, como $K_{\epsilon}^{C}$ é aberto, existe um ponto em $K_{\epsilon}^{C}$ que tem numero de rotação, mas isso, pelo corolario anterior implica o resultado. 


\section{Referências Bibliográficas}

[1] John Franks, Recurrence and fixed points of surface homeomoprhisms, Ergodic Theory and Dynamical Systems 8*, 99-107, 1988. 3, 6, 7, 54

[2] A. Katok; B. Hasselblatt, Introduction to the modern theory of dynamical systems, Cambridge University Press, 1995. 5

[3] S. Alpern; V. Prasad, Typical transitivity for lifts of rotationless annulus or torus homeomorphisms, London Math. Soc. 27, 79-81, 1995. vii, ix, 3

[4] Steve Alpern; V. Prasad, Typical recurrence for lifts of mean rotation zero annulus homeomorphisms, London Math. Soc. 23, 477-481, 1991. 3

[5] Fabio Armando Tal Salvador Addas-Zanata, Homeomorphism of the annulus with a transitive lift, Springer, 2009. 6

[6] Fabio Armando Tal, Rotation set and points with extremal velocities for regions of instability, 2011. 7

[7] Salvador Addas-Zanata Fabio Armando Tal, Homeomorphisms of th annulus with a transitive lift, arXiv:0811.3003v1 (math.DS) 18 Nov 2008, 2008. vii, ix, 7

[8] C. Conley; E. Zehnder, The birkhoff-lewis fixed point theorem and a conjecture of arnold, Invent Math. 73(1983),33-49, 1983. 3 